\title{
KELT: The Kilodegree Extremely Little Telescope
}

\author{
DISSERTATION \\ Presented in Partial Fulfillment of the Requirements for \\ the Degree Doctor of Philosophy in the \\ Graduate School of The Ohio State University \\ By \\ Joshua Pepper \\ $* * * * *$
}

The Ohio State University

2007

Dissertation Committee:

Approved by

Professor Richard Pogge, Adviser

Professor Krzysztof Stanek

Adviser

Professor B. Scott Gaudi

Adviser
Astronomy Graduate Program




\section{ABSTRACT}

The Kilodegree Extremely Little Telescope (KELT) project is an effort to find the best way to discover planetary transits of bright stars and to implement that method by building a telescope and discovering planets. In order to learn how to design a survey for transits of bright stars, we start by constructing a model of an all-sky survey for transits. That model points to an optimal survey configuration that uses a small-aperture, wide-field telescope to search for transits of stars in the range $8<V<10$ magnitude.

We used the parameters suggested by the model to build the KELT telescope, and we have deployed it to Winer Observatory in Arizona and begun a long-term survey for planets in a series of fields around the sky. The survey area covers about $25 \%$ of the Northern hemisphere and should allow us to detect the most scientifically valuable transiting planets. We examine the performance of the telescope with a number of metrics, and we find that it is performing at the level needed to detect the types of transits we are seeking.

We have completed the analysis of the first data set from KELT - a commissioning run from early 2005 that observed the Praesepe open cluster over 74 
nights. We obtained lightcurves of 69,337 stars, and detected 58 long period variables and 153 periodic variables. Sixteen of these are previously known variables, yielding 195 newly discovered variables for which we provide properties and lightcurves. We also searched for planetary-like transits, finding four transit candidates. Follow-up observations indicate that three of the candidates are astrophysical false positives, with one candidate inconclusively characterized. 
Dedicated to Bohdan Paczynski, my first adviser. 


\section{ACKNOWLEDGMENTS}

I would like to thank all those who helped me through this dissertation. My advisers Rick Pogge, Scott Gaudi, and Kris Stanek have done so much to teach me how to be a scientist. My co-authors on the papers that became this work were invaluable in completing this research, including Andy Gould, Darren DePoy, Amy Stutz, Shawn Poindexter, Rob Siverd, Jenn Marshall, Tom O'Brien, and Greg Sivakoff. My work with other members of this department has helped me overcome many challenges, and I would like to thank Chris Burke, Marc Pinsonneault, Chris Onken, Chris Morgan, Deokkeun An, and Don Terndrup for many helpful discussions. At Winer Observatory, Mark and Pat Trueblood have spent countless hours making sure that the KELT telescope runs smoothly and have made this project possible.

The Digitized Sky Surveys were produced at the Space Telescope Science Institute under U.S. Government grant NAG W-2166. The images of these surveys are based on photographic data obtained using the Oschin Schmidt Telescope on Palomar Mountain and the UK Schmidt Telescope. The plates were processed into the present compressed digital form with the permission of these institutions. 
The National Geographic Society - Palomar Observatory Sky Atlas (POSS-I) was made by the California Institute of Technology with grants from the National Geographic Society.

The Second Palomar Observatory Sky Survey (POSS-II) was made by the California Institute of Technology with funds from the National Science Foundation, the National Geographic Society, the Sloan Foundation, the Samuel Oschin Foundation, and the Eastman Kodak Corporation.

The Oschin Schmidt Telescope is operated by the California Institute of Technology and Palomar Observatory.

The UK Schmidt Telescope was operated by the Royal Observatory Edinburgh, with funding from the UK Science and Engineering Research Council (later the UK Particle Physics and Astronomy Research Council), until 1988 June, and thereafter by the Anglo-Australian Observatory. The blue plates of the southern Sky Atlas and its Equatorial Extension (together known as the SERC-J), as well as the Equatorial Red (ER), and the Second Epoch [red] Survey (SES) were all taken with the UK Schmidt.

All data are subject to the copyright given in the copyright summary. Copyright information specific to individual plates is provided in the downloaded FITS headers. 
Supplemental funding for sky-survey work at the STScI is provided by the European Southern Observatory.

This work was supported by grant NNG04GO70G from the National Aeronautics and Space Administration issued through the Origins of Solar Systems program. 


\title{
VITA
}

\author{
September 29, 1977 ......... Born - Cleveland, Ohio \\ $2000 \ldots \ldots \ldots \ldots \ldots \ldots \ldots \ldots$ A.B. Astrophysical Sciences, Princeton University \\ $2000-2001$.............. Answerthink, Inc. New York, NY \\ Consultant - Business Intelligence Services \\ $2001-2007$.............. Graduate Teaching and Research Associate, \\ The Ohio State University
}

\section{PUBLICATIONS}

\section{Research Publications}

1. J. Pepper, "Results from the KELT Transit Survey", AAS, 209, 226.01, (2006).

2. M. S. Peeples, A. Z. Bonanos, D. L. DePoy, K. Z. Stanek, J. Pepper, R. W. Pogge, M. H. Pinsonneault, and K. Sellgren, "The Nature of the Variable Galactic Center Source GCIRS 16SW Revisited: A Massive Eclipsing Binary", ApJL, 654, L61, (2006).

3. J. Pepper and C. J. Burke, "Survey for Transiting Extrasolar Planets in Stellar Systems IV: Variables in the Field of NGC 1245", AJ, 132, 1177, (2006).

4. J. Pepper and B. S. Gaudi, "Towards the Detection of Transiting Hot Earths and Hot Neptunes in Galactic Open Clusters", AcA, 56, 183, (2006).

5. B. S. Gaudi and J. Pepper, "Toward the Detection of Transiting Hot Earths and Hot Neptunes in Open Clusters", Protostars and Planets V, 1286, 8512, (2005). 
6. J. Pepper and B. S. Gaudi, "Searching for Transiting Planets in Stellar Systems", ApJ, 631, 581, (2005).

7. D. L. DePoy, J. Pepper, R. W. Pogge, A. Stutz, M. Pinsonneault, and K. Sellgren, "The Nature of the Variable Galactic Center Source IRS16SW", ApJ, 617, 1127, (2004).

8. A. Gould, J. Pepper, and D. L. DePoy, "Sensitivity of Transit Searches to Habitable Planets", ApJ, 594, 533, (2003).

9. J. Pepper, A. Gould, and D. L. DePoy, "Using All-Sky Surveys to Find Planetary Transits", AcA, 53, 213, (2003).

\section{FIELDS OF STUDY}

Major Field: Astronomy 


\section{TABle of Contents}

Abstract ........................... ii

Dedication ............................ iv

Acknowledgments . . . . . . . . . . . . . . . . . . . v

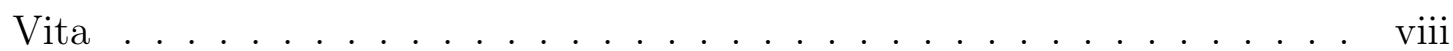

List of Tables . . . . . . . . . . . . . . . . . . . . . . xiii

List of Figures . . . . . . . . . . . . . . . . . . . . xv

Chapter 1 Introduction . . . . . . . . . . . . . . . . . . . 1

1.1 Searching for Extrasolar Planets . . . . . . . . . . . . . . . . . . . 1

1.2 Planetary Transits . . . . . . . . . . . . . . . . . . . 2

1.3 Transit Surveys . . . . . . . . . . . . . . . . . . . . 5

1.4 Known Transiting Planets . . . . . . . . . . . . . . . . . . . 6

1.5 The KELT Project $\ldots \ldots \ldots \ldots \ldots \ldots \ldots$

Chapter 2 Theoretical Background: Using All-Sky Surveys to Find Planetary Transits . . . . . . . . . . . . . . . . . . 10

2.1 Introduction . . . . . . . . . . . . . . . . . . . . . . . 10

2.2 Scaling Relations . . . . . . . . . . . . . . . . . . . . . . . . 11

2.3 Random Noise . . . . . . . . . . . . . . . . . . . . . . . . . . . 17

2.4 Number of Systems Probed $\ldots \ldots$. . . . . . . . . . . . 20

2.5 Implications For Telescope Design . . . . . . . . . . . . . . . 22 
2.5.1 Considerations for Aperture Size . . . . . . . . . . . . 24

2.5.2 Optimal Telescope Design . . . . . . . . . . . . . . . . 29

2.5.3 Practical Implementation . . . . . . . . . . . . . . . . . 31

Chapter 3 KELT Instrumentation and Performance . . . . . . . . . . 37

3.1 Introduction . . . . . . . . . . . . . . . . . . . 37

3.2 KELT System Overview . . . . . . . . . . . . . . . . . . . . 38

3.2 .1 Instrument f . . . . . . . . . . . . . . . . . . . . 38

3.2 .2 Observatory Site . . . . . . . . . . . . . . . . . 42

3.2 .3 Observing Operations . . . . . . . . . . . . . 43

3.2.4 Data Handling and Archiving . . . . . . . . . . . . . 46

3.2 .5 Data Reduction . . . . . . . . . . . . . . . . . 48

3.3 Instrument Performance . . . . . . . . . . . . . . . . . . . . 51

3.3.1 Telescope Performance . . . . . . . . . . . . . . . . . . . 51

3.3.2 Astrometric Performance . . . . . . . . . . . . . . . . . . 52

3.3 .3 Image Quality . . . . . . . . . . . . . . . . . . . . . . 54

3.3.4 Photometric Sensitivity . . . . . . . . . . . . . . . 56

3.4 Relative Photometry . . . . . . . . . . . . . . . . . . . 58

3.4.1 Difference Imaging Performance . . . . . . . . . . . . . . . 59

Chapter 4 KELT Commissioning Observations: A Photometric Survey for Variables and Transits in the Field of Praesepe . . . . . . . 73

4.1 Introduction . . . . . . . . . . . . . . . . . . . . 73

4.2 Observations . . . . . . . . . . . . . . . . . . 74

4.3 Data Reduction . . . . . . . . . . . . . . . . . . . . . 76

4.3 .1 Changing FWHM . . . . . . . . . . . . . . . . 76 
4.3.2 Image Subtraction . . . . . . . . . . . . . . . . . . . 79

4.3.3 Astrometry and Matching to Known Sources . . . . . . . . . . 80

4.3 .4 Rescaling Errors . . . . . . . . . . . . . . . . . . . . 81

4.3.5 Photometric Precision . . . . . . . . . . . . . . . . . . . 82

4.4 Variable Selection . . . . . . . . . . . . . . . . . . . 83

4.4 .1 Stetson Statistic . . . . . . . . . . . . . . . . . . . 83

4.4 .2 Variable Clustering . . . . . . . . . . . . . . . . 85

4.4.3 Identification of Long-Period Variables . . . . . . . . . . 87

4.4 .4 Periodicity Search . . . . . . . . . . . . . . . . . . 88

4.5 Transit Search . . . . . . . . . . . . . . . . . . . . . . . . . . . . . 89

4.5 .1 Detrending . . . . . . . . . . . . . . . . . . 90 90

4.5 .2 Transit Selection . . . . . . . . . . . . . . . . . . . 91

4.6 Variable Stars . . . . . . . . . . . . . . . . . . . . . . 93

4.6 .1 Matching to Known Variables . . . . . . . . . . . . . . . . 93

$4.6 .2 \quad$ Variable Star Catalog . . . . . . . . . . . . . . . . 95

4.7 Transit Candidates . . . . . . . . . . . . . . . . . . . . . 96

Chapter 5 Discussion . . . . . . . . . . . . . . . . 149

5.1 Results of Testing and Commissioning Phases . . . . . . . . . . . . 149

5.2 Main KELT Survey . . . . . . . . . . . . . . . . . . . . . . 150

5.3 Current Status of KELT and Future Work … . . . . . . . . . 152

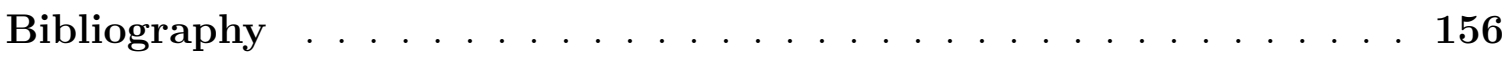




\section{List OF TABLES}

4.1 Variable Stars Identified in KELT Observations of Praesepe: Positions and 2 MASS Data . . . . . . . . . . . . . . . . . . 121

4.2 Variable Stars Identified in KELT Observations of Praesepe: Variability 135 


\section{LiST OF FiguRES}

2.1 Transiting systems $F\left(M_{V}\right)$ probed for fixed planetary radius and semimajor axis as a function of $M_{V} \ldots \ldots \ldots \ldots \ldots$

2.2 Distribution of highest $\Delta \chi^{2}$ values for different observing schedules. . $\quad 36$

3.1 Calculated response function of the KELT CCD camera and filter. . . 62

3.2 Pixel scale for the KELT $200 \mathrm{~mm}$ lens. . . . . . . . . . . . . . 63

3.3 Pixel scale for the KELT $80 \mathrm{~mm}$ lens. . . . . . . . . . . . . . . . . 64

3.4 Stellar PSFs from the $200 \mathrm{~mm}$ lens. . . . . . . . . . . . . . . . . 65

3.5 Stellar PSFs from the $80 \mathrm{~mm}$ lens. . . . . . . . . . . . . . . . 66

3.6 Contours of FWHM for stellar images for a $200 \mathrm{~mm}$ image. . . . . . . 67

3.7 Contours of FWHM for stellar images for a $80 \mathrm{~mm}$ image. . . . . . . . 68

3.8 Contours of $D_{80}$ for stellar images for a $200 \mathrm{~mm}$ image. . . . . . . . . 69

3.9 Contours of $D_{80}$ for stellar images for a $80 \mathrm{~mm}$ image. . . . . . . . . 70

3.10 RMS plot for one night of data from the $200 \mathrm{~mm}$ lens. . . . . . . . . 71

3.11 RMS plot for eight nights of data from the $80 \mathrm{~mm}$ lens. . . . . . . . . 72

4.1 RMS vs. $R_{K}$ magnitude for KELT stars. . . . . . . . . . . . . . 100

4.2 Lightcurves of three LPVs. . . . . . . . . . . . . . . . . . . . . 101

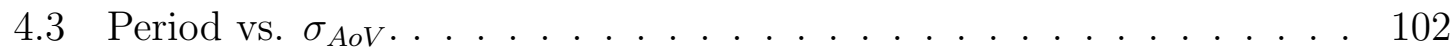

4.4 RMS plot before and after application of SYSREM detrending. . . . . 103

4.5 Sample lightcurve before and after SYSREM detrending. . . . . . . . 104

4.6 Color-magnitude diagram for cluster stars and field stars. . . . . . . . 105 
4.7 Color-magnitude diagram for variable and non-variable stars. . . . . . 106

4.8 Lightcurves of pulsating periodic variables. . . . . . . . . . . . . . 107

4.9 Lightcurves of pulsating periodic variables. . . . . . . . . . . . . . . . 108

4.10 Lightcurves of pulsating periodic variables. . . . . . . . . . . . . . . 109

4.11 Lightcurves of pulsating periodic variables. . . . . . . . . . . . . . . . 110

4.12 Lightcurves of eclipsing periodic variables. . . . . . . . . . . . . . . . 111

4.13 Lightcurves of eclipsing periodic variables. . . . . . . . . . . . . . . . 112

4.14 Lightcurves of eclipsing periodic variables . . . . . . . . . . . . . 113

4.15 Lightcurves of eclipsing periodic variables. . . . . . . . . . . . . . . . 114

4.16 Lightcurves of eclipsing periodic variables. . . . . . . . . . . . . 115

4.17 Lightcurves of eclipsing periodic variables . . . . . . . . . . . . 116

4.18 Lightcurves of eclipsing periodic variables. . . . . . . . . . . . . 117

4.19 Lightcurves of eclipsing periodic variables. . . . . . . . . . . . . . . 118

4.20 Lightcurves of eclipsing periodic variables. . . . . . . . . . . . . . 119

4.21 Transit candidates. . . . . . . . . . . . . . . . 120

5.1 Map of KELT survey fields. . . . . . . . . . . . . . . . . 154

5.2 Distribution of KELT survey observations across time. . . . . . . . 155 


\section{Chapter 1}

\section{INTRODUCTION}

\subsection{Searching For Extrasolar Planets}

Starting with the discovery of planets outside our solar system around the pulsar PSR1257+12 (Wolszczan \& Frail 1992), the search for extrasolar planets has progressed along a number of different fronts. In 1995, the first planet orbiting a

main sequence star was discovered around 51 Pegasi (Mayor \& Queloz 1995). To date over 220 extrasolar planets have been discovered using a variety of methods. Each method can be used to find a different kind of planet or target a different planetary environment. Gravitational microlensing is the only method with the potential to find free-floating planets, and can probe planets with low masses and those orbiting at several Astronomical Units (AU) from their host stars. Pulsar timing can also probe for very low planet masses, but naturally can only find planets around stellar remnants. Astrometry is a promising discovery method, but must await the launch of missions that can achieve the needed micro-arcsecond astrometric precision such as the Space Interferometry Mission. The radial-velocity (RV) method has proven the most productive search technique in terms of the number of planets found, 
but it, too, has some limitations. It can find planets with masses down to several earth masses, with orbital periods up to decades, but is generally limited to probing late-type unevolved stars, and as a discovery method it is limited to fairly bright stars, since the spectroscopic integration times become prohibitively long for faint stars.

The remaining major method for exoplanet discovery is the transit method, which has both great potential and serious difficulties. However, its greatest strength is in the ability to discover planets with the greatest scientific potential for individual analysis. In the next section I shall describe the power of searching for planets via transits.

\subsection{Planetary Transits}

The term "planetary transits" refers to the phenomenon of a planet crossing the line of sight between Earth and a star, blocking some fraction of the light from the star. For centuries, the only times these events were observed was when Venus or Mercury passed across the face of the Sun. In modern times, it was proposed that this method could be used to discover planets around other stars (Rosenblatt 1971) by photometrically monitoring many stars and searching for a slight dimming of the starlight characteristic of a transit. 
The basic physics of transits are fairly straightforward. When a planet transits its star, it causes the light from the star to dim by a factor of $(r / R)^{2}$, where $r$ is the radius of the planet and $R$ is the radius of the star. It is immediately clear that this effect will be stronger for larger planets and smaller stars. However, a planet can only be discovered through this method if the orbital plane of the planet is aligned to within a few degrees of our line of sight. Assuming that $\cos (i)$ is randomly distributed, where $i$ is the inclination of the orbit with respect to the line of sight from Earth, the probability that a planet will transit its star is $R / a$, where $a$ is the semimajor axis of the planetary orbit.

Transit searches have both advantages and disadvantages compared with RV searches. While RV surveys can only search the brightest stars in the sky (typically $V<9$ mag), transit surveys can probe stars down to much fainter magnitudes (The OGLE survey has found transits around stars with $V=16.5 \mathrm{mag}$ ). Transit surveys can therefore search many more stars than RV surveys, with some transit surveys monitoring over a million stars, while RV surveys usually observe a few thousand stars at most.

On the other hand, because of the inclination selection effect, only a small fraction $R / a$ of the stars with planets will actually transit their host stars as seen from Earth, meaning that a transit survey will only be able to detect that small subset of planets. For close-in planets (those with an orbital period $P$ less than 10 days) orbiting solar-type stars, $R / a \approx 10 \%$, meaning that a transit survey will miss 
$90 \%$ of such planets, and even more of the longer-period planets. An RV search by comparison can detect planets orbiting at a much larger range of inclinations. Also, while RV searches are able to select the specific stars to observe, most transit surveys are structured to observe all of the stars in a given field, which means that a significant fraction of the stars in the sample will be background giants. Giants are not useful to probe for planets using transits since their large radii causes the size of the transit signal to drop by the square of their increased radius, but since they are so bright they constitute a large fraction of the stars in a magnitude-limited survey.

These are just some of the considerations that impact the yield of transit searches. However, the most important advantage of transit surveys is that the planets they discover actually transit, while the vast majority (98\%) of planets found through RV surveys do not transit their host stars. (That percentage is higher than the $90 \%$ of non-transiting close-in planets since RV searches have probed out to much larger semimajor axes than the close-in regime.) The radius of a non-transiting planet cannot be determined, and therefore neither the size nor the density can be known. Also, RV measurements cannot typically provide the planet's mass, but only $m \times \sin (i)$, where $m$ is the planet's mass. Since the inclination of a non-transiting planet cannot generally be derived from just RV observations, only a minimum mass can be calculated for the planet. Follow-up observations of a transiting planet can fully characterize the planet's mass and radius, and therefore the planet's density. 
Even more importantly, planets that transit bright stars can be investigated for many astrophysically interesting phenomena. These transits provide the opportunity to study the internal structure of planets (Guillot 2005), their atmospheric composition (Charbonneau et al. 2002), spin-orbit alignment (Gaudi \& Winn 2007), the planet's oblateness (Hui \& Seager 2002), and can probe for the presence of rings or moons (Barnes \& Fortnoy 2004). This fact has been amply demonstrated by the discovery and analysis of the transiting planet HD209458b (Henry et al. 2000; Charbonneau et al. 2000; Cody \& Sasselov 2002).

\subsection{Transit Surveys}

There have been two main types of transit surveys carried out during the past decade. The first type is the so-called "pencil beam" survey. These have small fields of view but use relatively deep exposures to observe many faint stars. Pencil-beam surveys fall into two basic classes: field star surveys (Howell et al. 2000; Mallen-Ornelas et al. 2001; Udalski et al. 2002a) that observe selected regions of the Milky Way, and cluster surveys (Street et al. 2000; Burke et al. 2002) that target gravitationally bound star clusters sharing a common age and distance from Earth. While pencil-beam surveys are potentially capable of establishing the frequency of planets in various environments, they are unlikely to find transits of bright stars that would be most useful for intensive follow-up analysis. Although some of the 
surveys of field stars are considered "wide field", their total survey areas are small compared to $4 \pi$ steradians. An alternative method to the pencil beam surveys is a shallow wide-field survey that observes many bright stars over a wide area. These surveys do not reveal as much about planets in different environments or population statistics - rather they provide the best opportunity to find transiting planets that are well-suited for intensive follow-up observations that tell us more about the planets themselves.

Wide-field transit surveys are designed to observe one or more fields on the sky many times over the course of a long observing campaign. The resulting light curves of the stars observed are sampled sporadically rather than continuously, since data is only taken at night, and part or all of a night is often lost to bad weather or equipment problems. Therefore a long time baseline is necessary to build up enough signal to detect transits. Transit-like dips in the raw data stream are generally not obvious, but by repeatedly phase-folding the full light curve back on itself over a range of test periods and phases, one can obtain robust detections of transit events.

\subsection{Known Transiting Planets}

The first transiting planet was discovered by RV observations in 1999 (Mazeh et al. 2000), and was later found to be transiting (Henry et al. 2000; Charbonneau et al. 2000) in follow-up observations. The first planets discovered using the transit 
method were found by the OGLE survey (Udalski et al. 2002a,b,c, 2003, 2004), which observed the Galactic Bulge and was primarily intended to search for gravitational microlensing events. The OGLE-discovered planets are relatively faint, with host stars in the $14.9<V<16.6$ magnitude range. Surveys that are specifically designed to find transits of brighter stars have been started in the past seven years, using small aperture telescopes with wide fields of view. Four of these projects have discovered transits so far: two planets have been discovered by the Transatlantic Exoplanet Survey (TrES) (Alonso et al. 2004; O'Donovan et al. 2006); two by the XO project (McCullough et al. 2006; Burke et al. 2007); two by the Hungarian Automated Telescope (HAT) (Bakos et al. 2007a,b); and two by the SuperWASP project (Cameron et al. 2007). Two more planets that were initially discovered through RV surveys were subsequently found to be transiting (Bouchy et al. 2005; Sato et al. 2005), bringing the total number of transiting planets known to date (May 8, 2007) to sixteen.

\subsection{The KELT Project}

The aim of this dissertation has been to design, build, and deploy a telescope to take advantage of the potential of wide field transit surveys. The first step, described in Chapter 2, is to examine how to optimally design such an instrument. That examination starts by investigating the essential parameters needed for an all-sky 
survey to detect transits, and then developing a set of optimization parameters to design a telescope and detector system.

In Chapter 3 I present the details of the design, construction, deployment, and performance of the KELT telescope. The design of the KELT telescope and its survey strategy have been informed by the theoretical investigation outlined in Chapter 2. I describe regular telescope operations, how data is acquired and processed, and then talk about the planet-detection capabilities of the system.

In Chapter $4 \mathrm{I}$ describe and analyze the commissioning campaign using KELT to observe the Praesepe star cluster, which was used to construct the data reduction process. I discuss details unique to that data set and how they were dealt with, and then I present the discovery of new variable stars in the data and describe the search for transits.

In Chapter 5 I summarize what was accomplished and learned from this dissertation work and I describe the current status and next steps for the main KELT survey and its search for planets.

Most of the material in this study has appeared in previously published form or is in the process of publication. Chapter 2 was published by J. Pepper, A. Gould, and D. L. DePoy as "Using All-Sky Surveys to Find Planetary Transits" in Acta Astronomica, Volume 53, page 213, in 2003. 
Chapter 3 has been submitted by J. Pepper, R. W. Pogge, D. L. DePoy, J. L. Marshall, K. Z. Stanek, A. M. Stutz, S. Poindexter, R. Siverd, T. P. O’Brien, M. Trueblood, and P. Trueblood as "The Kilodegree Extremely Little Telescope (KELT): A Small Robotic Telescope for Large-Area Synoptic Surveys" to the Astronomical Journal.

Chapter 4 will be submitted by J. Pepper, K. Z. Stanek, R. W. Pogge, D. L. DePoy, R. Siverd, S. Poindexter, and G. Sivakoff as "A Photometric Survey for Variables and Transits in the Field of Praesepe with KELT" to the Astronomical Journal. 


\section{Chapter 2}

\section{Theoretical Background: Using All-Sky Surveys to Find Planetary Transits}

\subsection{INTRODUCTION}

In order to examine the performance of a wide-field transit survey, we need to first look at theoretical models to see how to optimally configure such a survey. In constructing these models, we will begin by assuming an all-sky survey. This starting point is useful for two reasons. First, it is the simplest scenario that we can build upon to investigate more specific strategies later. Second, there are several all-sky surveys already being planned for objectives other than transit detections. It should be possible, for instance, to utilize the photometric data stream of upcoming astrometric missions for transit detection. Space-based projects such as GAIA ${ }^{1}$ will take hundreds of observations of millions of stars over the course of years with the aim of obtaining precise astrometry. These data could equally well be analyzed for planetary transits.

\footnotetext{
${ }^{1}$ http://gaia.esa.int/
} 
There are also several existing or proposed ground-based all-sky surveys, including the Large-aperture Synoptic Survey Telescope (LSST) ${ }^{2}$ and the Panoramic Survey Telescope and Rapid Response System (PAN-STARRS)로. These surveys will be imaging the entire sky every few days, a qualitatively similar cadence to that of GAIA.

In this chapter, we examine the process of analyzing photometric data streams from all-sky surveys to find transits. We calculate the detection sensitivity, the distance out to which these detections will be possible, and the number of false-positive detections due to random noise. We derive a general expression for the number of stars that can be probed by this technique as a function of the total sensitivity of the survey. We apply our analysis to the problem of telescope design and conclude that very small, $5 \mathrm{~cm}$ telescopes are optimal for finding transits of bright stars.

\subsection{ScAling Relations}

In order to accurately determine the number of transits a given survey would be expected to detect, we must carefully define the set of stellar systems that can be probed for transits by the survey. This will evidently depend on the number density $n$, the luminosity $L$, and the radius $R$, of the stars being probed; as well as the

\footnotetext{
${ }^{2}$ http://www.lsst.org/

${ }^{3}$ http://pan-starrs.ifa.hawaii.edu/public/
} 
semi-major axis $a$ and radius $r$ of their planets. A quick (and naive) formulation would then state that the total number of systems that can be probed for transits $N_{p}$ is

$$
N_{p}=\frac{4}{3} \pi n \frac{R}{a}\left[d_{\max }(L, R, a, r)\right]^{3}
$$

for a homogeneous population of such stars, where $d_{\max }$ is the distance out to which a transit can be detected.

However, the quantity $d_{\text {max }}$ is ill-defined. For fixed $L, R, a, r$ and distance $d$, the transit detection scales as $\left(1-x^{2}\right)^{1 / 2}$ where $x$ is the transit impact parameter normalized to $R$; that is, $0 \leq x \leq 1$. Hence, the signal-to-noise ratio scales as $\left(1-x^{2}\right)^{1 / 4}$. Since detections normally require a minimum $\mathrm{S} / \mathrm{N}, d_{\max }$ must also be a function of the impact parameter $x$. Thus, for a photon-noise limited survey, $d_{\max } \propto\left(1-x^{2}\right)^{1 / 4}$, and therefore

$$
\frac{d N_{p}}{d x}=\frac{4}{3} \pi n \frac{R}{a}\left[d_{\max }(L, R, a, r, x)\right]^{3}
$$

where $d_{\max }(L, R, a, r, x)=d_{\max }(L, R, a, r, 0)\left(1-x^{2}\right)^{1 / 4}$. Here $d_{\max }(L, R, a, r, 0)$ is the distance out to which a transit can be detected for an edge-on $\left(i=90^{\circ}\right)$ orbit. We must then integrate over all values of the impact parameter $x$ from 0 to 1 , and so

$$
N_{p}=\int \frac{d N_{p}}{d x}=\frac{4 \pi n R}{3 a} \eta\left[d_{\max }(L, R, a, r, 0)\right]^{3}
$$


where,

$$
\eta=\int_{0}^{1}\left(1-x^{2}\right)^{3 / 4} d x=\frac{\sqrt{\pi}}{2} \frac{\Gamma(7 / 4)}{\Gamma(9 / 4)} \approx 0.719
$$

We now determine the dependence of $N_{p}$, the total number of systems probed, on the remaining parameters $L, R, a$, and $r$. To do so, we analyze the detection requirement,

$$
N_{t}\left(\frac{\delta}{\sigma}\right)^{2} \geq \Delta \chi_{\min }^{2}
$$

where $N_{t}$ is the number of observations of the transit over the length of the survey, $\delta$ is the fractional change in the star's brightness during the transit, $\sigma$ is the fractional error of an individual flux measurement, and $\Delta \chi_{\min }^{2}$ is the minimum acceptable difference in $\chi^{2}$ between a fit that assumes a constant flux and one that takes account of a transit. As we discuss in $\S 2.3, \Delta \chi_{\min }^{2}$ must be set sufficiently high to avoid spurious detections due to random noise.

- To determine the dependence of $N_{p}$ on $L$, we note that in equation (2.3), the only factor that depends on $L$ is $d_{\text {max }}^{3}$. For a particular star, the flux $f=L /\left(4 \pi d^{2}\right)$, thus $d \propto L^{1 / 2}$, and so $N_{p} \propto L^{3 / 2}$.

- For the dependence of $N_{p}$ on $R$, we note that $f \propto d^{-2}$, and that $\sigma \propto f^{-1 / 2}$, so $d \propto \sigma$. From equation (2.5), we see that $\sigma \propto \delta N_{t}^{1 / 2}$. Since $N_{t}$ is the total number of observations of the star during transits over the length of the survey, 
$N_{t}=N_{\text {obs }}(2 R) /(2 \pi a)$. Also, $\delta=(\pi r)^{2} /(\pi R)^{2}$. Thus, $d_{\text {max }} \propto R^{-3 / 2}$. Combining

these relations with the explicit factor of $R$ in equation (2.3) itself, we arrive at $N_{p} \propto R^{-7 / 2}$.

- For the dependence of $N_{p}$ on $a$, we see that $d \propto \sigma$, and $\sigma \propto \delta N_{t}^{1 / 2}$. Using $N_{t} \propto a^{-1}$, we have $d_{\text {max }}^{3} \propto a^{-3 / 2}$, and so $N_{p} \propto a^{-5 / 2}$.

- Finally, for the dependence of $N_{p}$ on $r$, the only factor that depends on $r$ is $\delta$, from $\delta=(\pi r)^{2} /(\pi R)^{2}$. Thus $\sigma \propto r^{2}$, and so $N_{p} \propto r^{6}$.

Consolidating the dependence of $N_{p}$ on the various parameters, we finally arrive at,

$$
N_{p}=\frac{4}{3} \pi n \eta d_{0}^{3}\left(\frac{R_{0}}{a_{0}}\right)\left(\frac{L}{L_{0}}\right)^{3 / 2}\left(\frac{R}{R_{0}}\right)^{-7 / 2}\left(\frac{a}{a_{0}}\right)^{-5 / 2}\left(\frac{r}{r_{0}}\right)^{6}
$$

where $d_{0}=d_{\max }\left(L_{0}, R_{0}, a_{0}, r_{0}, 0\right)$. That is, $d_{0}$ is the distance out to which a planet-star system with $i=90^{\circ}$ and the fiducial parameters $L_{0}, R_{0}, a_{0}, r_{0}$ can just barely be detected at the $\mathrm{S} / \mathrm{N}$ threshold.

We now seek to simplify equation (2.6) by integrating over the local stellar population at fixed absolute magnitude, $M_{V}$, and so we replace the three independent variables $(n, R, L)$ by the single variable $M_{V}$. We consider two regimes $M_{V} \geq 6$ and $M_{V} \leq 6$ (with one overlapping bin at $M_{V}=6$, which we will use later to check for consistency). 
We first treat the fainter regime. Here, the main sequence is relatively narrow. Hence, $R$ may be regarded as a function of $L$ (and so, therefore, of $M_{V}$ ), while $n$ is simply the number density of stars in a given magnitude bin. Hence, the "integration" amounts to a simple multiplication of factors.

We adapt the number density of stars $n$ from the empirically determined local stellar luminosity function (LF): for the range $\left(9 \leq M_{V} \leq 18\right)$ we use the LF reported in Zheng et al. (2001), and for the range $\left(6 \leq M_{V} \leq 8\right)$ we use the LF of Bessell \& Stringfellow (1993).

To estimate stellar radii, we combine the linear color-magnitude relation, $M_{V}=3.37(V-I)+2.89$ from Reid (1991), a color/surface-brightness relation $\log \left(R / R_{\odot}\right)=0.69+0.2226(V-K)-0.2 M_{V}$, based on the data of van Belle (1999), and VIK color-color relations for dwarfs from Bessell \& Brett (1988). We can therefore calculate the relative number of systems with a fixed $a$ and $r$ as a function of $M_{V}$, which we designate

$$
F\left(M_{V}\right)=\left[\frac{n\left(M_{V}\right)}{n_{0}}\right]\left[\frac{L\left(M_{V}\right)}{L_{0}}\right]^{3 / 2}\left[\frac{R\left(M_{V}\right)}{R_{0}}\right]^{-7 / 2}
$$

where $n_{0}, L_{0}$, and $R_{0}$ are the normalizations chosen below.

For the upper main sequence, $M_{V} \leq 6$, we evaluate $F\left(M_{V}\right)$ directly using the Hipparcos catalog (ESA 1997). For example, the LF for $M_{V}=4$ would be computed by summing $\sum_{i}\left[(4 / 3) \pi D_{i}^{3}\right]^{-1}$ over all stars within the Hipparcos completeness limit, 
$V<7.3$, having $3.5<M_{V}<4.5$, and lying within 50 pc. The distance $D_{i}$ is the minimum of $50 \mathrm{pc}$ and the distance at which the star would have $V=7.3$. Actually, we are not directly interested in the LF at $M_{V}=4$, but rather in the integral of $L^{3 / 2} R^{-7 / 2}$ over the subpopulations that make up the $M_{V}=4$ bin of the LF. Hence

$$
F\left(M_{V}=4\right)=\sum_{3.5<M_{V, i}<4.5}\left(\frac{L_{i}}{L_{0}}\right)^{3 / 2}\left(\frac{R_{i}}{R_{0}}\right)^{-7 / 2}\left(\frac{4}{3} \pi n_{0} D_{i}^{3}\right)^{-1} .
$$

The stellar radii are determined from Hipparcos/Tycho $\left(B_{T}, V_{T}\right)$ photometry and the color/surface-brightness relation of Gould \& Morgan (2003), $\log R / R_{\odot}=0.597+0.536\left(B_{T}-V_{T}\right)-M_{V_{T}} / 5$, ultimately derived from van Belle (1999).

To normalize $F$, we adopt the values associated with $M_{V}=5$ stars: $L_{0}=0.86 L_{\odot}, R_{0}=0.97 R_{\odot}, n_{0}=0.0025 \mathrm{pc}^{-3}$.

The resulting function (Fig. 2.1) shows that the majority of stars that are probed will be $\mathrm{F}$ and $\mathrm{G}$ type $\left(2 \lesssim M_{V} \lesssim 6.5\right)$. To check how this distribution depends on the size of the volume sampled, we recalculate the distribution function for the case in which the observed stars cover a much larger volume - one which would be better described by a thin disk, rather than a spherically uniform distribution. For this case we find that the scalings shown in equation (2.7) are replaced by $F\left(M_{V}\right) \propto n h L R^{-2}$, where $h$ is the scale height of each population. The distribution function is still dominated by F and G stars, but there are more $\mathrm{K}$ and early to mid 
M stars $\left(7 \lesssim M_{V} \lesssim 12\right)$. Of course, it is common knowledge that magnitude-limited $\left(F \propto n L^{3 / 2}\right)$ samples of main-sequence stars will be dominated by $\mathrm{F}$ and $\mathrm{G}$ stars. The interesting feature of Figure 2.1 is that this result does not qualitatively change despite the addition of the factor $R^{-7 / 2}$ in equation (2.7), which very strongly favors later-type stars.

\subsection{RANDOM NOISE}

Equation (2.6) describes what kinds of exoplanetary systems can be detected by a certain survey, given a photometric detection limit. The threshold $\Delta \chi_{\min }^{2}$ is determined by taking account of the fact that the data stream from an transit search must be analyzed for any combination of the parameters $R, a$, and $r$ within reasonable ranges. Such analysis will, however, yield a number of false-positive detections due to random noise. The threshold value of $\Delta \chi_{\min }^{2}$ must be chosen to yield a manageable number of candidate systems for follow-up observations.

To determine $\Delta \chi_{\min }^{2}$, we generate 1000 independent simulated streams of photometric observations of a single system with a host star of one solar mass and a circular planetary orbit. We attempt to simulate a schedule that would be characteristic of an all-sky survey that re-images a given star approximately every few days with varying intervals between observations. For each data stream, we generate 1000 observations at irregular intervals over 1800 simulated days, and then 
phase the observations for a range of periods from 3.0 to 3.1 days and a range of transit lengths. The number of the observations and the duration of the simulated survey are arbitrarily chosen as plausible characteristics for the type of all-sky survey we envision.

The duration of the transit depends on the orientation of the system with respect to our line of sight, and we analyze the data for eight equally spaced, progressively more inclined orientations. For each orientation, we test for 16 equally spaced phases. We test the resulting data sets for transit-like dips in the light curve, which we define simply as intervals during which the local mean light curve dips significantly below the global average. This $(8 \times 16)$ grid structure is chosen empirically: we find that the number of false positives increases linearly with grid density below this density and then flattens above it.

The result shows that for this kind of system, in order to restrict follow-up analysis to the $0.1 \%$ of the full sample most likely to yield a true transit detection, the value for $\Delta \chi_{\min }^{2}$ should be set to $\sim 36.6$. We take the highest value of $\Delta \chi^{2}$ from each of the 1000 sets, and of those highest values, we then sort the 1000 sets from highest value of $\Delta \chi^{2}$ to lowest. In Fig. 2.2 (inset), we plot the highest value of $\Delta \chi^{2}$ for each of the 1000 data streams.

To check the robustness of this number, we run additional simulations of 25 data sets, each with different observation schedules. First, we generate a set of 
observations that are randomly distributed throughout a 1800 day mission, with the requirement that no two observations be less than 10 minutes apart. Then we regenerate the data set with observations that are evenly spaced throughout the project. We also conduct the analysis on data sets with evenly spaced pairs of observations, with the observations in each pair separated by 14.4 minutes; and then again with the pair-spacing at 43.2 minutes.

These different configurations test for different types of observing schedules. For instance, a ground-based all-sky survey would most likely observe a star at essentially random times throughout a project. On the other hand, a space-based mission with a slowly rotating telescope (similar to the Hipparcos satellite) would observe a star in pairs of observations separated by minutes. For various reasons there could be a certain regularity imposed on either the space-based or ground-based observations.

We find that the value for $\Delta \chi_{\min }^{2}$ does not depend strongly on the observing schedule. We determine this by taking the highest value of $\Delta \chi^{2}$ for each of the 25 independent data streams, sorting the highest values of $\Delta \chi^{2}$ from each set in the manner described above, and then plotting the results for each type of observing schedule (Fig. 2.2). We find that there is only a $\sim 10 \%$ variation in $\Delta \chi_{\min }^{2}$ for different schedules.

The value of $\Delta \chi_{\min }^{2}$ depends on the number of different parameter configurations that are tested. When we rerun our analysis using a period range of 3.0 to 3.5 
days, we increase the size of our parameter space by a factor of 5 . We expect that the value of $\Delta \chi_{\min }^{2}$ depends on the size of the parameter space, $\Psi$, according to $\Delta \chi_{\min }^{2}\left(\Psi_{\text {new }}\right)-\Delta \chi_{\min }^{2}\left(\Psi_{\text {old }}\right)=2 \ln \left(\Psi_{\text {new }} / \Psi_{\text {old }}\right)$. This prediction agrees with the simulations, which show that an increase in parameter space by a factor of 5 leads to an increase in $\Delta \chi_{\min }^{2}$ by $2 \ln (5)=3.2$.

We then predict $\Delta \chi_{\min }^{2}$ if the size of the parameter space is expanded to search for transits with periods between 2 and 10 days (the range of periods most probable for the sort of fiducial values of the other parameters we have chosen). This would increase $\Psi$ by a factor of 80 compared to the period range of 3.0 to 3.1 days. Therefore, $\Delta \chi_{\min }^{2}$ for the expanded range of periods is higher than 36.6 by $2 \ln (80)=8.8$. So the value expected for $\Delta \chi_{\min }^{2}$ for such an analysis is about 45 .

\subsection{Number of Systems Probed}

The weak dependence of $\Delta \chi_{\min }^{2}$ on the observing schedule implies that the sensitivity of a project to planets essentially depends only on the total number of photons detected from each star, and not on the details of how they are collected. For a given star, this number is obviously proportional to the flux. We therefore characterize the sensitivity of the observing setup (telescope + detectors + duration + weather + etc.) by $\gamma$, the total number of photons that are detected from a fiducial $V=10 \mathrm{mag}$ star during the entire project. (Here we adopt $V=10$ as a 
reference, although the stars of interest lie in the range $8 \lesssim V \lesssim 10$.) We can utilize the various relations used to derive equation (2.6) to relate $\gamma$ to $V_{\max }$, the maximum apparent magnitude at which an equatorial transit can be detected,

$$
V_{\max }=-2.5 \log \left(\frac{\Delta \chi_{\min }^{2} \pi a R^{3}}{r^{4} \gamma}\right)+10
$$

Then, considering equations $(2.5),(2.6)$, and $(2.7)$, we obtain

$$
N_{p}=730 F\left(M_{V}\right)\left(\frac{a}{a_{0}}\right)^{-5 / 2}\left(\frac{r}{r_{0}}\right)^{6}\left(\frac{\gamma}{\gamma_{0}}\right)^{3 / 2}\left(\frac{\Delta \chi_{\min }^{2}}{45}\right)^{-3 / 2}
$$

where we have adopted $\gamma_{0}=1.25 \times 10^{7}, a_{0}=10 R_{\odot}, r_{0}=0.10 R_{\odot}$, and where we have made our evaluation at $M_{V}=5$ (i.e. $R=0.97 R_{\odot}, V_{\max }=10, d_{0}=100 \mathrm{pc}$, and $\left.n=0.0025 \mathrm{pc}^{-3}\right)$. Note that $\gamma_{0}=1.25 \times 10^{7}$ corresponds to approximately 625 20-second exposures with a $5 \mathrm{~cm}$ telescope and a broadened $(V+R)$ type filter for one $V=10$ mag fiducial star.

As mentioned above, a noteworthy feature of equation (2.10) is that $N_{p}$ depends on the characteristics of the survey primarily through the parameter $\gamma$. Moreover, since $\Delta \chi_{\min }^{2}$ depends only logarithmically on the size of the parameter space being explored, it plays a minimal role in survey design compared to the other variables in equation (2.10).

We envision two scenarios to which these results will be applicable. In one, a stream of photometric measurements from a space-based astrometric mission, 
such as GAIA, could be searched for transits. In the other, a ground-based survey could use one or more dedicated telescopes to search all bright stars in the solar neighborhood.

\subsection{Implichtions For Telescope Design}

We now apply the general analysis of $\S 2.2$ and $\S 2.4$ to the problem of optimizing telescope design for quickly locating a "large" number of bright $(V \lesssim 10)$ transiting systems. Since only one such systems is now known, we define "large" as $\mathcal{O}(10)$. From equation $(2.10)$ and the $0.75 \%$ frequency of hot jupiters measured from RV surveys, there are roughly 5 such systems to be discovered over the whole sky per magnitude bin at $M_{V}=5$ for $V_{\max }=10$. Hence, from Figure 2.1, of order 25 are to be discovered from all spectral types. It would, of course, be possible to discover even more by going fainter, but setting this relatively bright limit is advisable for three reasons. First, as we argued in the introduction, the brightest transits are the most interesting scientifically, and most of the transits detected in any survey will be close to the magnitude limit. Second, as we discuss below, a high dynamic range, $\Delta V=V_{\max }-V_{\min }$, can only be achieved at considerable cost to the observing efficiency. Hence, if high efficiency is to be maintained, setting $V_{\max }$ fainter means eliminating the brightest (most interesting) systems. Third, at $V_{\max }=10$, we are 
already reaching distances of 100 pc for $\mathrm{G}$ stars. Hence the number of transits observed in fainter surveys will not continue to grow as $d_{0}^{3}$ as in equation (2.6).

In previous sections, we ignored the loss of sensitivity to systems that are brighter than $V_{\min }$, which is set by saturation of the detector (or more precisely, by the flux at which detector non-linearities can no longer be accurately calibrated). This fraction is $10^{-0.6 \Delta V}$, or $6 \%$ for $\Delta V=2$, which we therefore adopt as a sensible goal. That is, we wish to optimize the telescope design for,

$$
8=V_{\min }<V<V_{\max }=10 .
$$

(In any event, essentially all stars $V<8$ have already been surveyed for exoplanets using RV, and the problem of determining which among the planet-bearers have transits is trivial compared to the problem of conducting an all-sky photometric variability survey.)

Optimization means maximizing the photon collection rate, $\gamma / T$, where $T$ is the duration of the experiment and $\gamma$ is, again, the total number of photons collected from a fiducial $V=10$ mag star. Explicitly,

$$
\gamma=K \mathcal{E} T D^{2} \frac{(\Delta \theta)^{2}}{4 \pi},
$$

where $\Delta \theta$ is the angular size of the detector, $D$ is the diameter of the primary-optic, $\mathcal{E}$ is the fraction of the time actually spent exposing, and $K$ is a constant that 
depends on the telescope, filter, and detector throughput. For our calculations, we assume $K=K_{0} \equiv 40 e^{-} \mathrm{cm}^{-2} \mathrm{~s}^{-1}$, which is appropriate for a broad $(V+R)$ filter and the fiducial $V=10$ mag star. The design problems are brought into sharper relief by noting that $\Delta \theta=\mathcal{L} / D \mathcal{F}$, where $\mathcal{L}$ is the linear size of the detector and $\mathcal{F}$ is the focal ratio, or $f / \#$, of the optics. Equation (2.12) then becomes

$$
\gamma=\frac{K \mathcal{E} \mathcal{L}^{2} T}{4 \pi \mathcal{F}^{2}}
$$

That is, almost regardless of other characteristics of the system, the camera should be made as fast as possible. We will adopt $\mathcal{F}=1.8$, below which it is substantially more difficult to fabricate optics. A more remarkable feature of equation (2.13) is that all explicit dependence on the size of the primary optic has vanished: a $1 \mathrm{~cm}$ telescope and an $8 \mathrm{~m}$ telescope would appear equally good! Actually, as we now show, there is a hidden dependence of $\mathcal{E}$ on $D$, which favors small telescopes.

\subsubsection{Considerations for Aperture Size}

The global efficiency $\mathcal{E}$ can be broken down into two factors, $\mathcal{E}=\mathcal{E}_{0} \mathcal{E}_{S}$, where $\mathcal{E}_{0}$ is the fraction of time available for observing (i.e., during which the sky is dark, the weather is good, etc.), and $\mathcal{E}_{S}$ is the fraction of this available observing time that the shutter is actually open. The first factor is not affected by telescope design and so will be ignored for the moment. The second factor should be maximized. The 
smaller the telescope aperture is, the longer the exposures can be before a $V_{\min }=8$ mag star saturates. Since the readout time is fixed, a smaller fraction of time is lost to read-out. We adopt as benchmarks a detector with pixel size of $\Delta x_{p}=9 \mu \mathrm{m}$, well depths of $10^{5} \mathrm{e}^{-}$, and a telescope diameter of $5 \mathrm{~cm}$.

To make explicit calculations, $\theta_{\mathrm{PSF}}$, the full width at half maximum of the point spread function (PSF) must be specified. For the fast optics $(\mathcal{F}<$ few $)$ we consider here, the diffraction limit is always much smaller than a pixel, regardless of aperture: $\theta_{\text {diff }} / \theta_{p} \sim 1.22 \mathcal{F} \lambda / \Delta x_{p} \sim 0.16$ (for our fiducial choices). At the small apertures we will consider, the diffraction limit is larger than the seeing, so it is possible to make the PSF much smaller than a pixel, $\theta_{\mathrm{PSF}} \ll \theta_{p}$. This would have the advantage of reducing sky noise and is a useful approach when it is possible to always center the telescope at the same field position as is the case for "point and stare" experiments. However, for an all-sky survey, which cycles through many fields, such precision repeat pointing is extremely difficult. Without it, precision photometry is impossible unless the sub-pixel response of the CCD is mapped out in detail. We therefore adopt a Nyquist-sampled PSF, for which the sky noise is approximately that falling on $4 \pi \sim 13$ pixels.

Our overall consideration for telescope design must take into account three factors. First, we with to maximize observing efficiency $\mathcal{E}_{S}$. Second, we wish to achieve the highest possible signal-to-noise ratio. Third, we must avoid any distortion problems with the optics. There are four effects through which aperture size can 
impact these factors. Two of these effects, observing efficiency and scintillation noise, will drive us to larger telescopes, while the other two effects, sky noise and focal plane distortion, will drive us to smaller telescopes. As we show below, for the observing parameters we have specified an aperture of $5 \mathrm{~cm}$ ensures a manageable (and unique) balance between the various effects.

\section{Exposure Time vs. Readout Time}

Assuming Nyquist sampling, at most half the light from a point source falls within one pixel. We can directly calculate the ratio of time lost to readout $T_{\text {read }}$ to the time spent exposing $T_{\exp }$,

$$
\frac{T_{\text {read }}}{T_{\exp }}=1\left(\frac{D}{5 \mathrm{~cm}}\right)^{2} 10^{-0.4\left(V_{\max }-10\right)}\left(\frac{W}{W_{0}}\right)^{-1} \frac{K}{K_{0}} \frac{T_{\text {read }}}{30 \mathrm{~s}} 10^{0.4(\Delta V-2)}
$$

where $W$ is the well depth of the detector pixels, and $W_{0}=10^{5} \mathrm{e}^{-}$is a fiducial well depth. Note that the factor $10^{-0.4\left(V_{\min }-10\right)}$, which arises from the need to avoid saturation of the brightest stars (where $V_{\min }=V_{\max }-\Delta V$ ), has been broken up into two terms to permit easy comparisons of equation (2.14) with equations (2.16) and (2.18) below.

In order to maximize the efficiency $\mathcal{E}_{S}$, the fraction of observing time devoted to readout should be minimized, and therefore, according to equation (2.14), so should the aperture size. The telescope will operate reasonably efficiently so long as $T_{\text {read }} \lesssim T_{\text {exp }}$ 


\section{SCINTILlation}

Another concern that arises for small apertures is the effect of atmospheric scintillation, which is characterized by (Young 1967; Warner 1988),

$$
\frac{\Delta I}{I}=S_{0}\left(\frac{D}{\mathrm{~cm}}\right)^{-2 / 3} X^{3 / 2} \exp \left(-\frac{h}{h_{0}}\right)\left(\frac{2 T_{\exp }}{\mathrm{sec}}\right)^{-1 / 2}
$$

where $S_{0}=0.09, X$ is the airmass, $h$ is the altitude of the observatory, and $h_{0}=8$ $\mathrm{km}$ is the scale height of the atmosphere. Using values of $D=5 \mathrm{~cm}, X=1.5, h=2$ $\mathrm{km}$, and $T_{\exp }=30 \mathrm{~s}$, we find $\Delta I / I=0.0057$.

Hence, for exposure times set by a saturation threshold, $T_{\exp } \propto D^{-2}$ (see eq. $[2.14])$

$$
\frac{\text { Scintillation Noise }}{\text { Source Noise }}=1\left(\frac{D}{5 \mathrm{~cm}}\right)^{1 / 3} 10^{-0.2(V-10)} .
$$

Therefore, despite the common perception that scintillation is a greater problem for smaller telescopes, for the fixed photon counts per exposure that are of interest in the present context, scintillation noise increases with increasing aperture. However, this dependence is fairly weak. For $D=5 \mathrm{~cm}$, the scintillation noise is just slightly smaller than the photon noise at $V=10$. 


\section{SKY NOISE}

All the calculations in $\S \S 2.2,2.3$, and 2.4 have assumed that sky noise is negligible, i.e. $V \ll V_{\text {sky }}$ where $V_{\text {sky }}$ is the light from the sky falling on 13 pixels (for Nyquist sampling). Assuming a somewhat conservative mean sky brightness of $V=20.0 \mathrm{mag} / \operatorname{arcsec}^{2}$, one finds

$$
V_{\text {sky }}=10.6+5 \log \left[\frac{D}{5 \mathrm{~cm}} \frac{\mathcal{F}}{1.8}\left(\frac{\Delta x_{p}}{9 \mu \mathrm{m}}\right)^{-1}\right]
$$

To determine how much of a problem sky noise will be, we can compare it to the amount of photon noise,

$$
\frac{\text { Sky Noise }}{\text { Source Noise }}=\frac{3}{4}\left(\frac{D}{5 \mathrm{~cm}}\right)^{-1} 10^{0.2(V-10)} \text {. }
$$

Thus, sky noise is less than photon noise for a $5 \mathrm{~cm}$ telescope, but would become a serious problem for a substantially smaller aperture.

\section{Focal Plane Distortion}

Focal plane distortions toward the edge of the detector become difficult (and expensive) to correct when the field of view is too large. For example, for a $4 k \times 4 k$ detector with $\Delta x_{p}=9 \mu \mathrm{m}$ pixels,

$$
\Delta \theta=23^{\circ}\left(\frac{D}{5 \mathrm{~cm}}\right)^{-1}\left(\frac{\mathcal{F}}{1.8}\right)^{-1}
$$


Again, this is manageable for $D=5 \mathrm{~cm}$ but could would potentially be a problem for smaller apertures.

Although this field of view is not so large as to create focal plane distortions, it is important to note that for a field of view this large, the telescope must be placed on an equatorial mount. For the alternative, an alt-az mount, the rotation of the sky will cause stars at one edge of the field to move faster across the detector than stars at the opposite edge. For similar reasons, the telescope must track rather than using drift scan.

\subsubsection{Optimal Telescope Design}

The relationships described in $\S 2.5 .1$ through $\S 2.5 .1$ can be used to optimize the aperture $D$ for any survey parameterized by a given $V_{\max }$. One is driven to smaller apertures by the goals of minimizing scintillation noise and the fraction of time spent on readout, and to larger apertures by the goals of minimizing sky noise and field distortion. Given available $\mathcal{L}=3.6 \mathrm{~cm} 4096 \times 4096$ detectors and reasonably fast $\mathcal{F}=1.8$ optics, a $D=5 \mathrm{~cm}$ telescope is optimal for an all-sky survey of $V=10$ stars.

We use equation (2.13) to determine the required duration of the experiment using our optimally-designed telescope, adopting $\gamma=\gamma_{0}, K_{0}=40 \mathrm{e}^{-} \mathrm{cm}^{-2} \mathrm{~s}^{-1}$, $\mathcal{E}_{0}=20 \%, \mathcal{E}_{S}=50 \%, \mathcal{L}=3.6 \mathrm{~cm}$, and $\mathcal{F}=1.8$. The total time required to conduct 
the survey assuming only source photon noise is $T=4$ months. Taking into account sky noise and scintillation increases that time by a factor of 2.5 to 10 months.

For surveys that intend to search for transits of stars fainter than the range we consider in this paper $\left(8=V_{\min }<V<V_{\max }=10\right)$, the relations in $\S 2.5 .1$ through $\S 2.5 .1$ operate somewhat differently. For the magnitude range we are considering, equations (2.14) and (2.16) provide an upper limit on the aperture size, while equations (2.18) and (2.19) place a lower limit, in which both limits converge to $D=5 \mathrm{~cm}$. At fainter magnitudes, the aperture size is restricted according to equations (2.14) and (2.18):

$$
10^{0.2(V-10)} \lesssim \frac{D}{5 \mathrm{~cm}} \lesssim 10^{0.2\left(V_{\max }-10\right)}
$$

This equation shows how the aperture size limits combine into a single scaling relation. What of the other two limiting factors? The issue of focal plane distortions is unimportant at fainter magnitudes, since $\Delta \theta<23^{\circ}$, which is outside of the regime where such distortions are a factor. Scintillation effects are also not a factor at fainter magnitudes, since the scintillation-noise restriction requires that $(D / 5 \mathrm{~cm})<10^{0.6(V-10)}$, which is a looser restriction than that of equation $(2.20)$.

Therefore, for a survey of transits at a magnitude range $8=V_{\min }<V<V_{\max }=$ 10, the aperture size limits converge to $D=5 \mathrm{~cm}$, while at fainter magnitudes, the aperture size is given by equation (2.20). 


\subsubsection{PRACTICAL IMPLEMENTATION}

In the previous section, we estimated the duration of observations required to achieve the minimum $\mathrm{S} / \mathrm{N}$ to detect transits by hot Jupiters assuming certain fiducial parameters of a ground-based telescope, but calculated within the framework of a literal "all-sky" $(4 \pi)$ survey of randomly-timed observations that is more characteristic of satellite missions. The resulting estimate is useful for judging the viability of a given observing setup, but it glosses over a key issue in the detection of transits, namely the problem of folding the data. As discussed in $\S 2.3$, the number of folds (and hence the size of the search space that must be probed) scales directly as the duration of the experiment. This larger search space increases both the minimum $\Delta \chi^{2}$ for a robust detection and the amount of computing power needed to sift through the search space. The first effect is logarithmic in the size of the search space, so a 10 -fold increase changes $\Delta \chi_{\min }^{2}$ by only $\sim \ln 10 \sim 2.3$, which is well under 10\%. However, the second effect is linear in the search space and so could easily overwhelm available computing resources if not carefully monitored. That is, there are important drivers for keeping the duration of observations of any given field to a minimum and hence for exploring the question of whether it is better to break up the "all-sky" survey into several smaller components, each of which could be completed in a shorter time. Indeed, the OGLE experiment (Udalski et al. 2002a), the only transit experiment to successfully detect a transit (Konacki et al. 2003), 
was motivated by these considerations to concentrate its observations over a month duration so as to limit the number of foldings.

To estimate the true duration of the project required the achieve the minimum $\mathrm{S} / \mathrm{N}$, one must take account of two factors: First, as a practical matter, ground-based surveys from a single location can cover only an angular area $\Omega<4 \pi$. Second, during a year of continuous observations, any given patch of sky is observed only for about 6 months. These factors change the previous estimates from $\S 2.5 .2$. The first factor implies that the observing time required to reach minimum $\mathrm{S} / \mathrm{N}$ is actually lower than the time calculated from equation (2.13) by a factor of $\Omega / 4 \pi \sim 0.5$, since the project is observing about half the angular area on the sky. The impact of the second factor is more complex. In a given night, only half the accessible angular area $\Omega$ can be observed. Therefore, each night the available area to observe is lower by an additional factor of 2 , which means the rate of observations of a given point on the sky will double again. There are two scenarios at this point. The first scenario is that after accounting for the first factor, the time required for sufficient $\mathrm{S} / \mathrm{N}$ is less than a year. In this case, the two factors combine to lower the estimate from $§ 2.5 .2$ by a factor of about 4 . In the second scenario, the time required for sufficient $\mathrm{S} / \mathrm{N}$ is more than a year. In this case, after observing for 6 months the minimum $\mathrm{S} / \mathrm{N}$ is not reached, and therefore the project will have to pick up again six more months later when the target is again visible, and so the second factor does not apply. As 
we see below, however, for the parameters we are considering, we are well within the regime of the first scenario.

Applying the first factor to the result in $\S 2.5 .2$ reduces the calculated time from 10 months to 5 months. Since this latter duration is indeed smaller than 1 year, the second factor implies that the requisite $\mathrm{S} / \mathrm{N}$ will actually be reached in 2.5 months. This is only slightly longer than the duration of the OGLE observations.

However, real experiments inevitably have larger errors than expected. (We mention one source of additional errors below.) If the errors prove sufficiently larger that the experiment requires more than 1 year (after application of the first factor) to achieve the minimum $\mathrm{S} / \mathrm{N}$, then one would not gain the advantage of the second factor. In this case, it would be better to break the sky up into strips by declination, and observe each strip for a year, so as to increase the $\mathrm{S} / \mathrm{N}$ obtained in each strip during a single year, and so to permit the application of the second factor.

Another real-world consideration is that the photometric errors will not be Gaussian. For example, the Sloan Digital Sky Survey (SDSS) photometry errors, while Gaussian in their core, deteriorate to an exponential profile in the wings beginning at about $3 \sigma$ (Ivezic, Z et al. 2003). Such deterioration is likely to set in earlier for the small-aperture, wide-field, low-budget cameras that we envisage here. The non-Gaussian form of the errors has no practical impact on our analysis: there are so many data points that the central limit theorem guarantees that 
their combined behavior in each phase bin will be Gaussian (as we have implicitly assumed in $\S 2.3$. However, the non-Gaussian tails will tend to increase the $\sigma$ of the distribution relative to what would be inferred from the core, which of course will degrade the sensitivity of the experiment. If the errors are as well-behaved as those of SDSS, this problem can easily be resolved by the standard device of 3- $\sigma$ clipping. If not, then more complex strategies will be required. These are likely to be among the biggest practical problems facing the analysis, but in the absence of real data, they cannot be further analyzed here. 


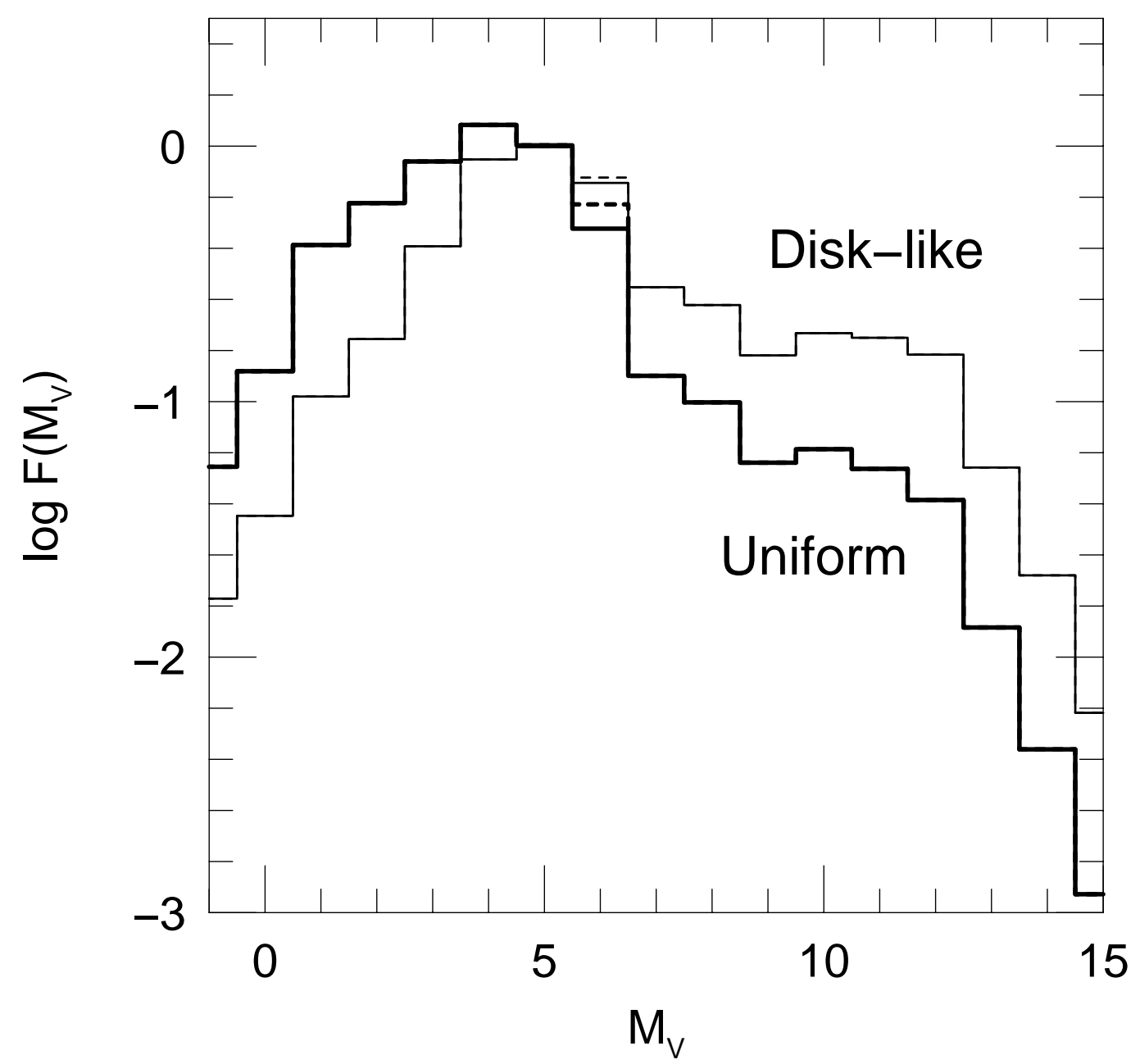

Fig. 2.1. - The relative number of potential transiting systems $F\left(M_{V}\right)$ probed for fixed planetary radius $r$ and semi-major axis $a$ as a function of $M_{V}$. The bold line applies to a uniform distribution of stars - to model the immediate solar neighborhood. The thin line applies to a thin disk - to model a search of a large portion of the Galactic disk. The dashed lines indicate where the two different methods for calculating the spatial density (as described in $\S 2.2$ ) overlap in each case. The distributions are arbitrarily scaled such that $F\left(M_{V}=5\right)=1$. 


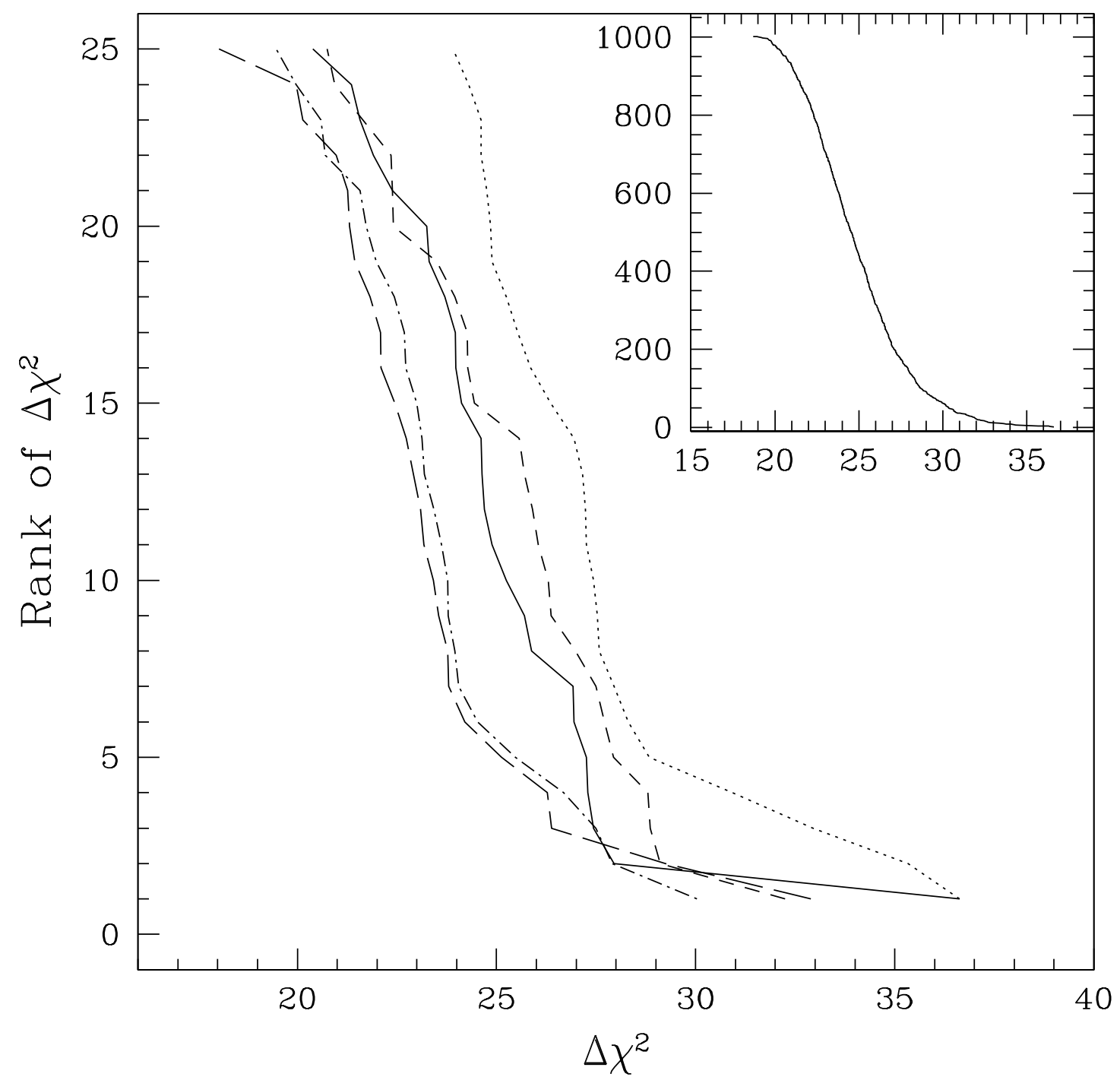

Fig. 2.2.- The main plot shows the highest $\Delta \chi^{2}$ values for each of 25 runs, plotted by rank, for each type of observing schedule. For four of the curves, a period search is conducted only for periods of 3.0 days to 3.1 days. Shown are a random schedule (solid), a regular schedule (short-dashed), a regular schedule with 14.4-minute pairspacing (long-dashed), a regular schedule with 43.2-minute pair-spacing (dot-dashed). The dotted line is the random schedule with a period range of 3.0 to 3.5 days. The inset plot shows the main simulation of 1000 data sets. The $0.1 \%$ highest value is at 36.6 . 


\section{Chapter 3}

\section{KELT Instrumentation AND PERFormance}

\subsection{INTRODUCTION}

In the last chapter, we investigated how to optimally design a transit survey to discover extrasolar planets around bright stars. We used that analysis to design, build, and deploy the Kilodegree Extremely Little Telescope (KELT) to meet those criteria. The system has two different observing configurations. The primary configuration is a "Survey Mode" designed for wide area coverage, either in large

strips or an all-sky survey, with the goal of covering broad sections of the sky with a large field of view, at a cadence of a few minutes on a nightly basis throughout most of the observing year. This mode implements the primary scientific driver of KELT and gives this telescope a wider field of view and targets a brighter magnitude range than other transit surveys of its kind. The second configuration is a "Campaign Mode" that uses a smaller field of view and is designed to conduct short duration intensive observing campaigns on specific fields. In Campaign Mode we have undertaken a 74-day campaign on the Praesepe open cluster. 
In this chapter, we describe the instrumentation, deployment, and operations of KELT (§3.2), we characterize the performance of the different components and the overall system in the field (§3.3), and we demonstrate our photometric precision $(\S 3.4)$.

\subsection{KELT System Overview}

\subsubsection{INSTRUMENT}

KELT consists of an optical assembly (CCD detector, medium-format camera lens, and filter) mounted on a robotic telescope mount. A dedicated computer is used to control the telescope, camera, observation scheduling, and data archiving system tasks. One goal in assembling KELT was to use as many off-the-shelf components and software packages as possible to speed the development.

The KELT detector is an Apogee Instruments 1 AP16E thermoelectrically cooled CCD camera. This camera uses the Kodak KAF-16801E front-side illuminated CCD with $4096 \times 40969 \mu \mathrm{m}$ pixels $(36.88 \times 36.88 \mathrm{~mm}$ detector area $)$ and has peak quantum efficiency of $\sim 65 \%$ at $600 \mathrm{~nm}$. The AP16E uses a PCI card and cable to control the camera and thermoelectric cooler (TEC). According to the camera specifications and confirmed by laboratory testing, the camera is operated at a conversion gain

\footnotetext{
${ }^{1}$ http://www.ccd.com
} 
of 3.6 electrons/ADU and delivers a measured readout noise of $\sim 15 \mathrm{e}^{-}$. The device is read out at 14 -bit resolution at $1.3 \mathrm{MHz}$, which gives a full-frame readout time of $\sim 30$ seconds. The CCD specifications claim a full-well depth of $\sim 100,000 \mathrm{e}^{-}$, but the 14-bit ADC saturates at $16383 \mathrm{ADU}\left(\sim 59,000 \mathrm{e}^{-}\right)$. The TEC can cool the device to $\sim 30^{\circ} \mathrm{C}$ below the ambient air temperature. Nominal dark current is $0.1-0.2 \mathrm{e}^{-}$pixel $^{-1} \mathrm{sec}^{-1}$ at an operating temperature of $-10^{\circ} \mathrm{C}$ (typical for $20^{\circ} \mathrm{C}$ ambient air temperature).

We use two different lenses with KELT. For the wide-angle survey mode, we use a Mamiya $64580 \mathrm{~mm}$ f/1.9 medium-format manual-focus lens with a $42 \mathrm{~mm}$ aperture. This lens provides a roughly $23^{\prime \prime} \mathrm{pix}^{-1}$ image scale and a $26^{\circ} \times 26^{\circ}$ field of view. To provide a narrow-angle campaign mode, we use a Mamiya $645200 \mathrm{~mm}$ f/2.8 APO manual-focus telephoto lens with a $71 \mathrm{~mm}$ aperture. This provides a roughly $9^{\prime \prime} .5 \mathrm{pix}^{-1}$ image scale and effective $10.8 \times 10^{\circ} .8$ field of view. Both lenses have some vignetting at the corners, and the image quality declines toward the outer part of the field, so the effective field of view is circular (see $\S 3.2 .5$ and $\S 3.3 .3$ for details).

To reject the mostly-blue background sky without greatly diminishing the sensitivity to stars (which are mostly redder than the night sky), we use a Kodak Wratten \#8 red-pass filter with a 50\% transmission point at $\sim 490 \mathrm{~nm}$ (the filter looks yellow to the eye), mounted in front of the KELT lens. The calculated response function of the KELT CCD and filter is shown in Figure 3.1. The transmission function for the Wratten \#8 filter is taken from the Kodak 
Photographic Filters Handbook (Kodak 1998), and the quantum efficiency curve for the Kodak KAF-16801E CCD was provided by the Eastman Kodak Company. The effect of atmospheric transmission on this bandpass is estimated for 1.2 airmasses at the altitude of Winer Observatory $(1515 \mathrm{~m})$ using the Palomar monochromatic extinction coefficients (Hayes \& Latham 1975), which are for an altitude of $1700 \mathrm{~m}$. We did not estimate in detail the atmospheric water vapor or $\mathrm{O}_{2}$ extinction terms, as these are not important for our application. The effective wavelength of the combined Filter+CCD response function (excluding atmospheric effects) is $691 \mathrm{~nm}$, with an effective width of $318 \mathrm{~nm}$, computed following the definition of Schneider, Gunn, \& Hoessel (1983). This results in an effective bandpass that is equivalent to a very broad R-band filter.

The optical assembly (camera+lens+filter) is mounted on a Paramount ME Robotic Telescope Mount manufactured by Software Bisque ${ }^{2}$. The Paramount is a research-grade German Equatorial Mount designed specifically for robotic operation with integrated telescope and camera control. According to manufacturer's ratings the periodic tracking error of the mount before correction is $\pm 5^{\prime \prime}$. That is smaller than the large pixels of the KELT camera and therefore does not affect our observations. The mount can carry an instrument payload of up to $75 \mathrm{~kg}$, more than sufficient for the KELT camera, which weighs approximately $9 \mathrm{~kg}$. The Paramount is installed on a stock $91 \mathrm{~cm}$ high steel pier using a stock base adapter plate. The

\footnotetext{
${ }^{2}$ http://www.bisque.com
} 
Paramount provides us with a robust, complete mounting solution for our telescope. The optical assembly is mated to the Paramount using a custom mounting bracket that mounts directly on the Paramount's Versa-Plate mounting surface.

The CCD camera and mount are controlled by a PC computer located at the observing site that runs the Windows XP Professional operating system and the Bisque Observatory Software Suite from Software Bisque. There are three main applications that we use for KELT: TheSky to operate the Paramount ME (pointing and tracking); CCDSoft CCD camera control software to operate the AP16E camera; and Orchestrate scripting and automation software to integrate the operation of TheSky and CCDSoft. Orchestrate provides a simple scripting interface that lets us control all aspects of a night's observing with a single command script. This software lets us prepare an entire night's observing schedule and upload it to the KELT control computer during the afternoon. A scheduled task on the computer starts up the system at sunset and loads the observing script into Orchestrate, after which the system runs unattended for the entire night, weather permitting.

The observing site provides AC power and Internet connectivity. To ensure clean AC power for the KELT telescope and control computer at the observing site (\$3.2.2) we use a 1500VA Powerware 9125 Uninterruptable Power Supply (UPS). This filters the line power and protects the system against surges or brief power outages. The control computer is connected to the Internet through the observing site's gateway, and its internal clock is synchronized with the network time servers at 
the Kitt Peak National Observatory using the Dimension 4 network time protocol (NTP) client ${ }^{3}$. This ensures sufficiently accurate timing for telescope pointing and time-series photometry. Since we observe at few-minute cadences, we can tolerate few-second timing precision, which is easily within the typical performance of Dimension 4 on the available $\mathrm{T} 1$ connection.

\subsubsection{Observatory Site}

The KELT telescope has been installed at the Irvin M. Winer Memorial Mobile Observatory ${ }^{4}$ near Sonoita, Arizona. Located at N $31^{\circ} 39^{\prime} 53^{\prime \prime}$, W $110^{\circ} 36^{\prime} 03^{\prime \prime}$, approximately 50 miles southeast of Tucson at an elevation of 4970 feet (1515 meters), Winer Observatory has a dedicated observing building with a $25 \times 50$-foot $(7.6 \times 15$ meter) roll-off enclosure and provides site and maintenance services. Winer currently hosts four robotic telescopes, including KELT. The KELT telescope pier is bolted to the concrete floor, and its control cables are run into a nearby telescope control room that houses the control computer and UPS. Winer also provides Internet access (currently via a dedicated DS/T1 line, but early in the project the site used a slower ISDN link), that allows us to remotely login to the control computer via a secure gateway.

\footnotetext{
${ }^{3}$ v5.0 from Thinking Man Software (www.thinkman.com/dimension4/)

${ }^{4}$ http://www.winer.org
} 
The weather conditions at Winer Observatory are roughly as good as comparable sites in southern Arizona; about $60 \%$ of all observing time is usable, with half of that time being measurably photometric. Since our PSFs are between 2 and 3 pixels, and the pixel scales are 9.5 and $23^{\prime \prime}$ (for the $200 \mathrm{~mm}$ and $80 \mathrm{~mm}$ lenses, respectively), atmospheric seeing variations (which are on the scale of arcseconds) are not a factor in our observations.

\subsubsection{ObSERVIng Operations}

Observations with KELT are carried out each non-clouded night using command scripts for pre-programmed, robotic operation; we do not undertake any remote real-time operations. The nightly observing scripts are created at Ohio State University (OSU) using a script-generation program written in Perl, and then uploaded to Winer Observatory where they are used by Orchestrate to direct the telescope to observe the specified fields for each night. Each night has a different script, and we generally upload scripts in 3-4 week batches during the main survey season, or more frequently during pointed-target campaigns.

The suite of software programs we use to control the telescope mount and camera works well, but has several limitations. Most importantly, the Orchestrate scripting package does not provide the built-in ability to program control loops 
or conditional branching, which is why we use a Perl program to create the Orchestrate scripts we upload to the control computer.

The scripts start the telescope each night based on the local clock time. On nights when the weather is judged to be good enough for observing, the observatory control computer automatically opens the roof of the observatory at nautical $\left(12^{\circ}\right)$ twilight and the telescope begins observations on schedule. If the weather is not suitable for observing, on-site personnel abort the command script. If the weather appears good at first but degrades during the night, the observatory computer closes the roof and the personnel abort the script. All data acquired are archived automatically at the end of the night.

When the script is loaded into Orchestrate, it first waits until one hour before astronomical $\left(18^{\circ}\right)$ twilight. At that point the telescope takes five dark images and five bias images, with the exposure times for the dark frames the same as the exposure times of the observations for the night. Once these calibration data are taken, the telescope goes back to sleep until astronomical twilight, at which point it slews to the first target field and begins the nightly observing sequence. Unless the weather turns bad, prompting the roof to close, observations continue until astronomical dawn. At this time the telescope is slewed to its stowed position, and five dark images and five bias images are acquired, ending observing for the night. (See $§ 3.2 .5$ for a discussion of KELT flatfields.) 
We have so far used KELT in two distinct operating modes: campaign mode, using the $200 \mathrm{~mm}$ lens, and survey mode, using the $80 \mathrm{~mm}$ lens. In campaign mode, the telescope intensively observes a single target field for an entire night. In survey mode, the telescope observes a number of fields that are equally spaced around the sky at $2^{h}$ intervals of Right Ascension centered on Declination $+31^{\circ} 39^{\prime}$ (the latitude of Winer).

In campaign mode, the observing script instructs the telescope to wait until the target field is above 2 airmasses, and to then observe the field continuously until it sinks below 2 airmasses or astronomical dawn, whichever happens first. In survey mode, the telescope begins observing after astronomical twilight, tiling between two fields at a time. New fields are observed as they rise above 1.4 airmasses. Provisions for Moon avoidance are built into the scripts to prevent observations of any survey field when it is within $45^{\circ}$ (two field widths from field center) of the Moon.

An operational complication arises because the KELT mount is a German Equatorial design. This means that fields observed East of the Meridian are rotated by $180^{\circ}$ relative to fields observed West of the Meridian. The practical effect is that we must separately reduce photometry for fields taken in East and West orientations, especially when we use difference-imaging photometry. To avoid the complication of creating two separate data pipelines to reduce data taken in survey mode, we instead observe only fields in the Eastern part of the sky. We lose some observation time due to periods when the Moon is within $45^{\circ}$ of all fields in the East above 1.4 
airmasses, leading to downtime when no field is available that meets the observing criteria. In those situations, the scripts instruct the telescope to pause observing until the next field becomes available. Overall, the loss to Moon avoidance reduces the total amount of data acquired by $\sim 10 \%$.

\subsubsection{Data Handling And Archiving}

Data acquired by the camera are immediately written to a hard drive in the control computer, logged, and then copied to one of two $250 \mathrm{~GB}$ external hard drives attached to the control computer by a USB interface. At the end of the night, all new images are automatically duplicated onto the other external hard drive. During long winter nights, the telescope can take as many as 500-600 images per night, depending on the exposure time, filling the drives every two weeks. Normally, however, bad weather and downtime due to Moon avoidance reduce the actual observing rate, so it typically takes 3-4 weeks for both storage drives to reach capacity.

Data quality is monitored daily using automated scripts running on the Windows observing computer at Winer. At the end of the night, a Perl script selects three images from the beginning, middle, and end of the night and uploads them to a computer at OSU. These sample images are analyzed for basic statistics: mean, median, and modal sky value and the mean Full-Width at Half Maximum (FWHM) 
of stars measured across the images, and then visually inspected to ensure that the camera and mount are operating correctly.

When the external drives approach full capacity, they are disconnected from the computer. One of the drives is a hardened drive made by Olixir Technologies (their Mobile DataVault) that serves as the transport drive. This drive is shipped via FedEx to the OSU Astronomy Department in Columbus, Ohio, in a cushioned transport box, as bandwidth limitations at Winer Observatory preclude online data transfer. The second drive is a conventional external USB drive without special mobile packaging made by Maxtor Corporation which serves as the backup drive and which is stored at Winer Observatory. Until the transport drive arrives at OSU and its data have been successfully copied and verified, the backup drive at Winer is stored and left idle. In the event a transport drive arrives damaged, data from the backup drive will be copied to another transport drive and shipped. In the meantime, the removed drives are replaced with two others of each type and operations continue. At any given time, we have four external disk drives in use: two drives in operation, one in transit, and one stored as a backup. For transferring hundreds of gigabytes of images every few weeks during the prime observing season, this procedure has proven to be very reliable and efficient. To date, out of dozens of drive shipments, we have lost only two drives to damage in transit, with no loss of data (both arrived damaged at Winer after their data were retrieved and copied at OSU). 
When a transport drive arrives at OSU, the drive is connected to our main data storage computer and all of the images are copied and run though a series of data quality checks. The image files are renamed, replacing the cumbersome default file name created by the CCDSoft application with a name indicating the field observed, the UTC date, and an image number. The images are then analyzed to measure image quality (modal sky and mean FWHM). If the modal sky value is above 800 ADU (due to moonlight, cloud cover, or other ambient light sources), a central square of $2000 \times 2000$ pixels is trimmed out of the center and compressed with gzip, with the original image discarded. The cutoff at 800 ADU was determined using two months of representative data showing that images compromised by excessive light contamination consistently had sky values above that level and were unsuitable for photometry. The cutoff is high, with many poor images well below the cutoff, but we choose to be conservative about eliminating images early in the reduction process. The images that pass the initial filter on sky values, and the trimmed sections of the bad images, are stored on a multi-terabyte RAID storage array at OSU, providing data redundancy and fast access for data reduction and analysis.

\subsubsection{DATA REDUCTION}

Here we describe the data reduction process in brief, for the purposes of evaluating the performance of the KELT camera. Detailed descriptions of the reduction process are presented in Chapter 4. 
The data reduction pipeline consists of three steps. First, we process the images by subtracting dark frames and dividing by a flatfield. Second, we identify all the stars in the field and determine their instrumental magnitudes. Third, we obtain the photometry on all images using difference image analysis.

Dark images are created for each night by median-combining 10 dark images - five from the beginning and end of each night. In early testing we determined that we can treat our dark frames as combined dark+bias. We take bias frames separately to monitor their stability, but do not incorporate them into the reduction process because the bias has been extremely stable. In cases where dark frames were not taken or there were problems with the images, we use good dark frames from nights bracketing the observations to create a substitute dark frame. We confirmed that using dark images from nearby nights did not significantly affect the statistics of the subtracted images - our dark images are quite stable from night to night.

The KELT system is challenging to accurately flatfield. For the $200 \mathrm{~mm}$ lens there is a combined decrease in flux of $\sim 18 \%$ between the center of the image and the edges, and a decrease of up to $\sim 26 \%$ between the center and the corners. For the $80 \mathrm{~mm}$ lens, the decrease is $\sim 23 \%$ from the center to the edge, and $\sim 35 \%$ from the center to the corners. Because of the large KELT field of view (10.8 and $26^{\circ}$ for the $200 \mathrm{~mm}$ and $80 \mathrm{~mm}$ lenses, respectively), twilight flats are not useful for flatfielding since the twilight sky is not uniform on those scales. Dome flats using a diffuse screen produced reasonable results with high signal-to-noise ratio. The flats 
are sufficiently repeatable that we do not need to regularly take dome flats. For relative photometry, the dome flats work adequately, and we are able to absolutely flatfield our images to $\sim 5 \%$ accuracy.

Once images have been dark-subtracted and flatfielded, we can then create catalogs of images and measure the brightnesses of stars on the images. This photometric analysis is done in two basic steps. The first is to create a high-quality reference image for a field by combining a few dozen of the best images and then use the DAOPHOT software package (Stetson 1987) to identify all of the stars in the field down to a faint magnitude limit and measure their approximate instrumental magnitudes. See $\S 3.4 .1$ for the details on how the instrumental magnitudes are calibrated to standard photometry.

Once a template and DAOPHOT star catalog with baseline instrumental magnitudes have been created, the second step is to process the images with the ISIS image subtraction package (Alard \& Lupton 1998; Alard 2000). Our reduction process is similar to that of Hartman et al. (2004). The ISIS package first spatially registers all of the images to align them with the reference image. The reference image is convolved with a kernel for each image and subtracted, creating a difference image. The flux for each star identified on the reference image is then measured on each subtracted image using PSF-fitting photometry. Image subtraction has been shown in limited tests to be equal to or better than other photometric methods for 
the purposes of transit searches (Bakos 2006). In section $\$ 3.4$ below, we provide additional information about the reduction process to obtain relative photometry.

\subsection{Instrument Performance}

In this section we quantify the performance of the KELT system by assessing in turn the telescope mount, the astrometric quality (geometric image quality), the image quality (position-dependent PSF), and photometric sensitivity.

\subsubsection{Telescope Performance}

Since the telescope was installed at Winer in October 2004, the hardware has performed up to specifications. There have been no significant problems with the mount or the control software. The pointing has not been perfect: our fields are so large that minor pointing errors do not significantly affect our scientific results, but during normal operations the typical intra-night drift is $\sim 25^{\prime}$ in Declination and $\sim 9^{\prime}$ in Right Ascension. We believe the drift is due to a slight non-perpendicularity between the orientation of the camera and the axis of the mount. While the magnitude of the drift seems large, it represents a movement of $\sim 65$ pixels, less than $2 \%$ of the size of the field. It does not cause stars to move across large portions of the detector, and therefore does not lead to significant changes in the PSFs of individual stars. Since our reduction method utilizes image subtraction, we do lose 
the ability to take good photometry at the edges of a field. However, because of PSF distortions and other effects (see §3.3.3), we already have degraded sensitivity in those regions. Therefore the loss of coverage and sensitivity due to drift is quite small. Future alignment of the telescope will attempt to reduce or eliminate the drift.

\subsubsection{Astrometric Performance}

Measurements of the positions of stars from the Tycho-2 Catalog (Høg et al. 2000) are used to determine the conversion between pixel coordinates and celestial coordinates on the KELT images. We use the Astrometrix ${ }^{5}$ package to compute polynomial astrometric solutions for our images following the procedure described by Calabretta \& Greisen (2002). To avoid stars that are saturated on the KELT images, we consider Tycho-2 stars with magnitudes $9.0 \leq V_{\text {Tyc }} \leq 10.0$. From these we select up to 1000 stars per image. A first attempt to compute a global astrometric solution for the entire $4096 \times 4096$ image produced large residuals for most of the outer parts of the detector, with discrepancies between the predicted and actual positions of Tycho-2 stars of many tens of pixels. Since our primary goal is to convert pixel coordinates into celestial coordinates on a star-by-star basis, a global solution is not required. We instead divide the image into 25 subimages on a $5 \times 5$ grid and perform a separate astrometric solution for each subimage. A third-order polynomial

\footnotetext{
${ }^{5}$ http://www.na.astro.it/\$\sim\$radovich/wifix.htm
} 
astrometric fit is computed for each subimage using Astrometrix. The individual subimage fits give much better results, with offsets between predicted and measured positions of catalog stars at the subpixel level except at the extreme corners of the field. The subframes overlap by a few tens of pixels, and fits to stars common to adjacent subimages are consistent at the arcsecond level. For the $200 \mathrm{~mm}$ lens, the typical RMS residuals are $\pm 00^{\prime \prime} 8$, or $<10 \%$ of the average pixel size of $\sim 99^{\prime \prime} 5$. The $80 \mathrm{~mm}$ lens has slightly worse RMS residuals, $\pm 5^{\prime \prime}$ or $\sim 20 \%$ of a pixel size of $22^{\prime \prime}$, but still well within tolerances for our purposes.

Having a good astrometric fit to the images permits a quantitative assessment of the geometric performance of the KELT optics. For the $200 \mathrm{~mm}$ lens, the effective pixel size decreases by about $1 \%$ from center-to-edge from 9.537 near the center to $9{ }^{\prime} 450$ at the edges of the field ( 9'.40 at the corners). Contours of constant effective pixel scale are circular and centered on the intersection of the optical axis of the $200 \mathrm{~mm}$ camera lens and the CCD detector, as shown in Figure 3.2. The square CCD pixels do not perfectly project onto squares on the sky, but slowly distort systematically away from the center, showing the characteristic signature of $\sim 0.5 \%$ pincushion distortion, expected for the manufacturer's typical claims for their telephoto lenses. As Figure 3.3 shows, for the $80 \mathrm{~mm}$ lens the effective pixel size decreases by about $3.5 \%$ from center-to-edge, from $23^{\prime \prime} .19$ near the center to $222^{\prime \prime} 40$ at the edges of the field (and $\sim 21^{\prime \prime} .8$ at the corners). This effect is larger than the one seen with the $200 \mathrm{~mm}$ lens, consistent with $\sim 2 \%$ pincushion distortion in this lens, 
typical of short focal-length wider-angle lenses. Contours of constant effective pixel scale are also circular and centered on the CCD detector.

The effect of the optical distortion is that pixels project onto smaller effective areas on the sky moving radially outward from the center of the CCD, making the sky appear non-flat (center-to-edge) at the $\sim 1.5 \%$ level for the $200 \mathrm{~mm}$ lens and at the $\sim 6 \%$ level for the $80 \mathrm{~mm}$ lens. There are two effects that act together to decrease the background sky level per pixel as you go radially outward from the center of the image: the decreasing pixel scale, and hence decreasing pixel area on the sky, with radius, and increasing vignetting with radius.

\subsubsection{IMAGE QUALITY}

As expected for such an optically fast system, the image PSF varies systematically as a function of position. The small physical pixel size $(9 \mu \mathrm{m})$ implies that the lens optics dominate the PSF, and we are insensitive to changes in atmospheric seeing. For both lenses, the systematic patterns in both the image full-width at half maximum (FWHM) and more refined measures of image quality (i.e. the $80 \%$ encircled energy diameter $D_{80}$ ) can be used to quantitatively assess the position-dependent image quality.

For the $200 \mathrm{~mm}$ lens, typical image PSFs have FWHM of $\sim 1.8-2.9$ pixels, and $80 \%$ encircled energy diameters of $D_{80}=4.7-9$ pixels. The $80 \mathrm{~mm}$ lens has a similar 
range of FWHM for stellar image PSFs, and the $80 \%$ encircled energy diameters range from $D_{80}=6-10$ pixels. There are significant changes in the detailed PSF shape across each image from the center to the extreme edges of the detector. Figure 3.4 shows representative stellar PSFs for a $5 \times 5$ grid across the CCD for the $200 \mathrm{~mm}$ lens. The $80 \mathrm{~mm}$ lens shows more pronounced distortions, as shown in Figure 3.5. Therefore, the $80 \mathrm{~mm}$ lens has a roughly $24^{\circ}$ diameter effective field of view with reasonably good images and little vignetting, whereas the $200 \mathrm{~mm}$ lens works well over most of the CCD detector except at the extreme corners.

Figures 3.6 and 3.7 show maps of the image FWHM as a function of position for the $200 \mathrm{~mm}$ and $80 \mathrm{~mm}$ lenses, respectively, derived from measurements of unsaturated, bright field stars in representative images. The most obvious feature in both is the strong vertical trend in increasing FWHM, with nearly no differences horizontally. Because this is seen with both lenses, which are of very different design, we believe this is because the CCD is tilted relative to the optical axis. Because the lens designs are proprietary, we do not know precisely how much the detector is tilted, nor the origin of the tilt at present. This effect could be due to how the detector is mounted inside the camera, or to the camera/lens mounting plate. This apparent field tilt also affects the maps of the $80 \%$ encircled-energy diameter $D_{80}$, shown in Figures 3.8 and 3.9 .

The $80 \mathrm{~mm}$ lens has very stable imaging performance over time. The FWHM and $D_{80}$ maps derived for images of the same field over a 11-month period show no 
significant changes. We have, however, periodically adjusted the focus when working with the telescope, which my cause some discontinuities in the data for the survey images. We will explore such effects in upcoming papers.

Unfortunately, the PSF is not stable across the image over time for the $200 \mathrm{~mm}$ lens. While intranight variations in the FWHM maps are quite small, there are significant changes from night to night that have no apparent correlation with hour angle, CCD temperature, or any other physical or environmental parameter for which we have measurements. The effect of the changes we see is for the region of best FWHM (the base of the trough seen in the FWHM map in Figure 3.6) to move vertically on the CCD by many hundreds of pixels. We do not yet know the cause. The main effect is to complicate the difference-imaging reductions of the data. We will discuss these complications and their mitigation in the subsequent paper describing our results for the $200 \mathrm{~mm}$ lens Praesepe cluster observing campaign.

\subsubsection{Photometric Sensitivity}

Given the nature of the KELT bandpass (see Figure 3.1), we calibrate our instrumental magnitudes to the $R$ band. We do so by rescaling our instrumental magnitudes by a constant, such that

$$
R_{K} \equiv-2.5 \log (\mathrm{ADU} / \mathrm{sec})+R_{K, 0}
$$


where the instrumental ADU/sec is measured using aperture photometry with IRAF, $R_{K}$ is defined as an approximate KELT $R$ magnitude, and $R_{K, 0}$ is the zero-point. We find that the $R_{K}$ magnitudes are within a few tenths of a magnitude of standard $R$ band photometry, with the uncertainty dominated by the color term. Since we do not have $V-I$ colors for all our stars, we quote observed magnitudes in $R_{K}$, which can be considered equivalent to Johnson $R$, modulo a color term defined by

$$
V=R_{K}+C_{V I}(V-I)
$$

where $C_{V I}$ is the $(V-I)$ color coefficient, and $V / I$ are in the Johnson/Cousins system. Since we do not have previously measured $R$ magnitudes of large numbers of stars in our fields in our magnitude range, we relate $R_{K}$ to known magnitudes by matching stars from our observations to the Hipparcos catalog, selecting only stars with measured $V$ and $I$ colors in Hipparcos. We take the mean instrumental magnitude from a set of high-quality images, and match the known magnitudes to the mean instrumental magnitudes, using Equations 3.1 and 3.2 .

For the cluster observations with the $200 \mathrm{~mm}$ lens, we select 22 calibration stars, and measure their instrumental magnitudes on 76 high-quality images, resulting in a magnitude zero-point of $R_{K, 0}=16.38 \pm 0.06 \mathrm{mag}$, and $C_{V I}=0.55 \pm 0.2$. For the survey observations with the $80 \mathrm{~mm}$ lens, we use 59 stars on 77 images, resulting in a magnitude zero-point of $R_{K, 0}=15.15 \pm 0.07 \mathrm{mag}$, and $C_{V I}=0.5 \pm 0.3$. Since the Hipparcos stars we use to calibrate our data have $(V-I)$ colors mostly between 
0 and 1 , we expect our calibrations to be less accurate for redder stars. These measured zero-point uncertainties suggest that the flatfield corrections are good to within $\sim 5 \%$ in absolute accuracy.

Tying together the full calibration process, we find that a fiducial $R=10$ mag star at the field center with $(V-I)=0$ has a flux of 356 counts per second with the $200 \mathrm{~mm}$ lens, and 115 counts per second with the $80 \mathrm{~mm}$ lens. Scaling those numbers by the different aperture sizes of the lenses $(71 \mathrm{~mm}$ aperture for the $200 \mathrm{~mm}$ lens and $42 \mathrm{~mm}$ aperture for the $80 \mathrm{~mm}$ lens), we find that the $200 \mathrm{~mm}$ lens is about $8 \%$ more efficient than the $80 \mathrm{~mm}$ lens.

\subsection{Relative Photometry}

KELT was designed primarily for precision time-series relative photometry (see Everett \& Howell (2001) for background). The crucial test for our instrument is the ability to obtain long-term lightcurves with low noise and minimal systematics. A simple test of KELT's photometric performance is to examine the root-mean-squared (RMS) of the magnitudes of an ensemble of lightcurves as a function of magnitude. We apply the ISIS image subtraction package to samples of our data to obtain relative photometry, and measure the statistics of the resulting lightcurves. Our criteria for the ability to detect planetary transits is the presence of substantial 
numbers of stars for which the RMS of the lightcurves are below the $2 \%$ and $1 \%$ levels.

\subsubsection{Difference Imaging Performance}

The instrumental magnitudes for the KELT lightcurves are produced through a combination of ISIS and DAOPHOT photometry. This process involves some careful conversion between DAOPHOT and ISIS flux measurements - see Appendix B of Hartman et al. (2004) for the details of the conversion. First, we create a reference image by combining a number of high-quality images. DAOPHOT measures the instrumental magnitude of the stars on the reference image $m_{i}(\mathrm{ref})$ based on PSF fitting photometry, with the magnitude of each star $i$ calculated from the flux by $m_{i}(\mathrm{ref}) \equiv 25-2.5 \log \left[f_{i}(\mathrm{ref})\right]+C_{a p}$, where $f_{i}$ is the flux measured by DAOPHOT and $C_{a p}$ is an aperture correction to ensure that $m_{i}($ ref $)=25-2.5 \log \left(c_{i}\right)$, where $c_{i}$ is the counts per second from the star in ADU. ISIS then creates an ensemble of subtracted images for the whole data set using the reference. To derive the full light curve, ISIS fits a PSF for each star on each subtracted image $j$, to obtain a flux $f_{i}(j)$. The DAOPHOT-reported instrumental magnitudes for the reference images serve as the magnitude baseline for the conversion of ISIS fluxes to magnitudes, where the magnitude of the $i$ th star on the $j$ th image is $m_{i}(j)=m_{i}(\mathrm{ref})-2.5 \log \left[1-f_{i}(j) / f_{i}(\mathrm{ref})\right]$. 
We then calculate the RMS variation of all the detected stars in both the Praesepe data set and for a sample of the survey data. Because of the night-to-night variations in the position of the best image quality on the detector with the $200 \mathrm{~mm}$ lens described at the end of $\S 3.3 .3$, we calculate the RMS for the Praesepe data from a single night of observations, to better demonstrate the intrinsic instrumental performance. In Figure 3.10 we plot the distribution of RMS versus $R_{K}$ magnitude for 67,674 stars on 32 images with 60 -second exposures from one night. With this lens and exposure time, we obtain photometry of stars in the magnitude range $R_{K}=8-16$ mag. For stars brighter than about $R_{K}=9.5$ mag, systematics begin to dominate the light curves, mostly due to saturation. Out of the 67,674 stars, 4,281 have RMS $<0.02 \mathrm{mag}$, and 1,369 have RMS $<0.01 \mathrm{mag}$.

We perform the same analysis for one of the regular survey fields observed with the $80 \mathrm{~mm}$ lens using 239 observations over 8 nights with 150-second exposures. We obtain photometry on 49,376 stars in the range $R_{K}=6-14 \mathrm{mag}$, and plot the data in Figure 3.11. We find 14,333 stars with RMS $<0.02 \mathrm{mag}$, and 3,822 stars with RMS $<0.01 \mathrm{mag}$, with systematics dominating for stars brighter than $R_{K}=7.5$ mag.

Overall, the RMS performance is mostly as expected from Poisson statistics except at the bright end. For the brightest stars in our data we see a floor in which the RMS no longer decreases as the stars get brighter, and instead becomes roughly constant at RMS $=0.004$ magnitudes. The RMS floor is indicative of a fixed pattern noise component, caused by intrapixel sensitivity on the CCD. The Kodak KAF-E 
series CCDs are 2-phase front-side illuminated devices in which the second poly-gate electrode on each pixel is a transparent gate made of Indium-Tin-Oxide (ITO) to boost the overall quantum efficiency of the device (Meisenzahl et al. 2000). Over much of the wavelength regime of interest for KELT, the ITO material is $\sim 2$ times more transparent than the regular silicon oxide material used on the first poly-gate. The result is significant pixel substructure in which the quantum efficiency varies stepwise across each $9 \mu \mathrm{m}$ pixel, which introduces a component of fixed-pattern noise that produces the observed RMS floor. We note that more recent models of commercial CCD cameras with the Kodak KAF-16801 detector are using a newer version of this device that incorporate a front-surface microlens array that mitigates the intrapixel step in transmission, but persons contemplating similar systems to our own should be aware of the issue and take it into account.

We do not expect to obtain high-precision photometry for the very brightest stars in our data, but the RMS floor is well below the $1 \%$ level and it should not significantly affect our ability to detect transits. We plot noise models in Figures 3.10 and 3.11, which include photon noise and sky noise, along with the RMS floor. In the future we will choose a lens focus that makes slightly larger FWHM images to minimize these effects. 


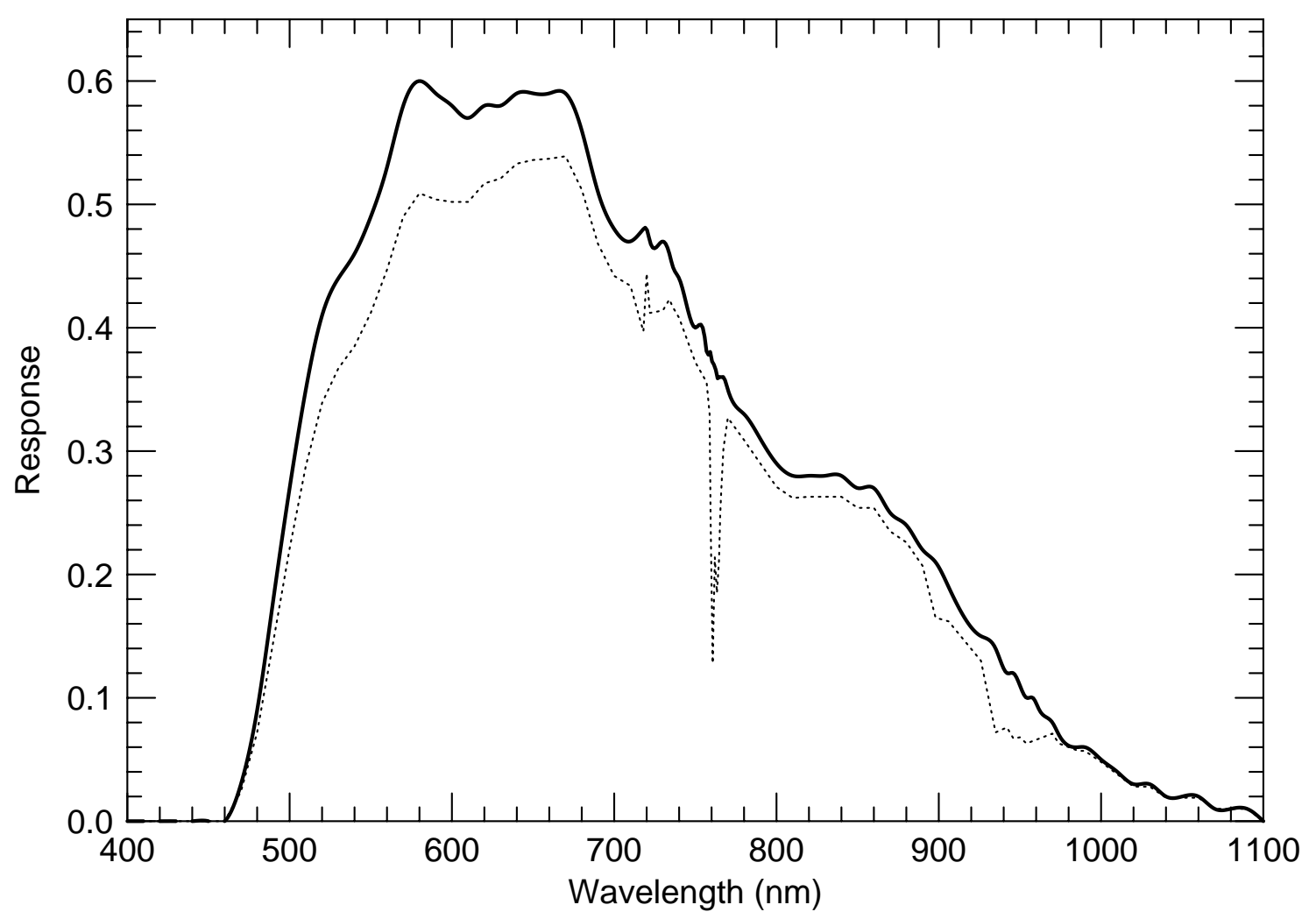

Fig. 3.1. - Calculated response function of the KELT CCD camera and Kodak \#8 Wratten filter. The dashed curve is the response function including atmospheric transmission at Winer Observatory for 1.2 airmasses. This response function does not include the transmission of the camera lenses. 


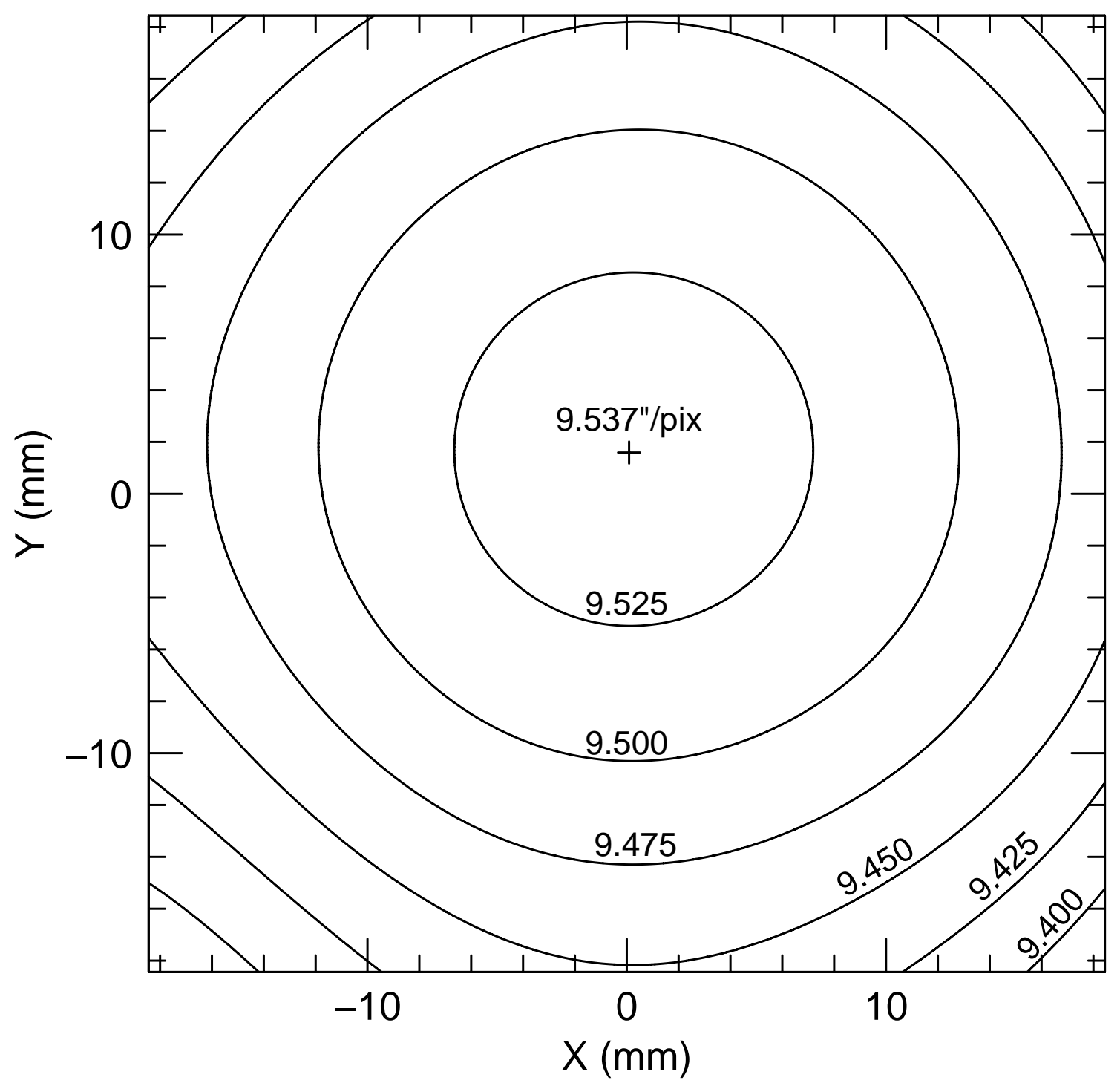

Fig. 3.2.- Effective pixel scale in arcseconds pixel $^{-1}$ for the KELT $200 \mathrm{~mm}$ lens. Contours show curves of constant effective pixel scale. The cross $(+)$ marks the optical center of the field, where the pixel scale is $9.537 \mathrm{pix}^{-1}$. 


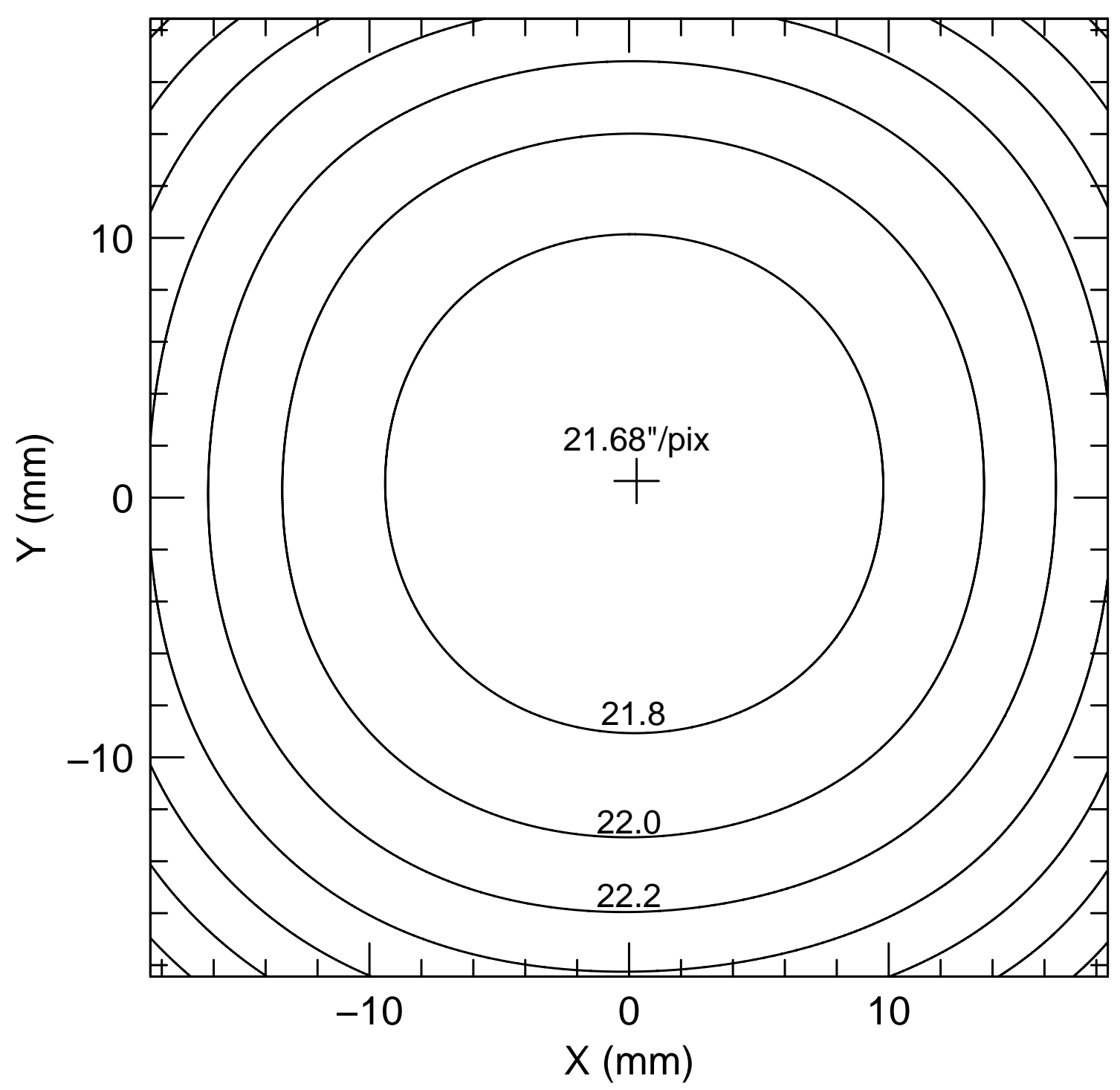

Fig. 3.3.- Effective pixel scale in arcseconds pixel ${ }^{-1}$ for the KELT $80 \mathrm{~mm}$ lens. Contours show curves of constant effective pixel scale. The cross $(+)$ marks the optical center of the field, where the pixel scale is $21^{\prime \prime} 68 \mathrm{pix}^{-1}$. 


\begin{tabular}{|c|c|c|c|c|}
\hline 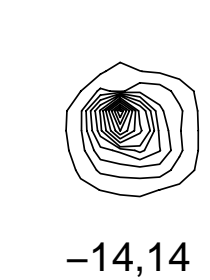 & 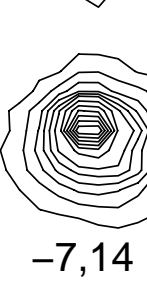 & 0,14 & 7,14 & $\underbrace{\circ}_{\sqrt{\sqrt{\sqrt{(14,14}}}}$ \\
\hline$-14,7$ & $-7,7$ & 0,7 & 7,7 & 14,7 \\
\hline$-14,0$ & $\begin{array}{l}\text { (e) } \\
-7,0\end{array}$ & $\begin{array}{c}50 \mu \mathrm{m} \\
0,0 \mathrm{~mm}\end{array}$ & 7,0 & 14,0 \\
\hline$-14,-7$ & $-7,-7$ & $0,-7$ & $7,-7$ & $14,-7$ \\
\hline$\circ-14,-14$ & -7,-14 & $0,-14$ & $7,-14$ & $14,-14$ \\
\hline
\end{tabular}

Fig. 3.4.- Representative stellar PSFs from the $200 \mathrm{~mm}$ lens, shown as intensity contours of bright, unsaturated stars taken with a $5 \times 5$ grid pattern on the CCD; the position of the center of each box, relative to the center of the image, is indicated in mm. Each box is 15 pixels $(135 \mathrm{~mm})$ on a side. The scale-bar in the center panel indicates $50 \mu \mathrm{m}$ on the detector (5.5 pixels). Contours show levels of $(5,10,15,20,25,30,40,50,60,70,80,90,100) \%$ of the peak intensity. 


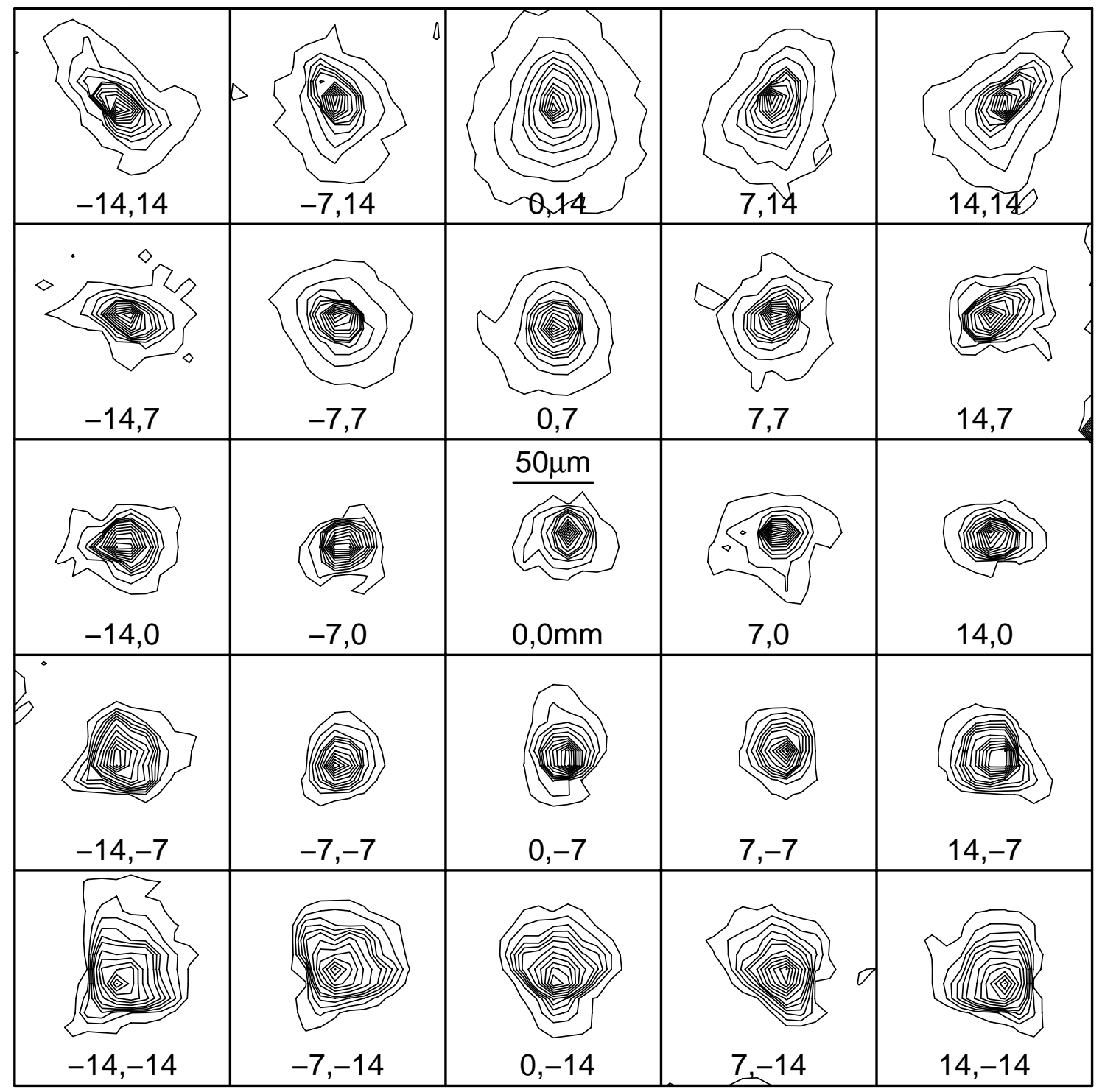

Fig. 3.5.- Representative stellar PSFs from the $80 \mathrm{~mm}$ lens, displayed in the same format as in Figure 3.4. The images are more severely distorted at the extreme edges of the field than with the $200 \mathrm{~mm}$ lens. 


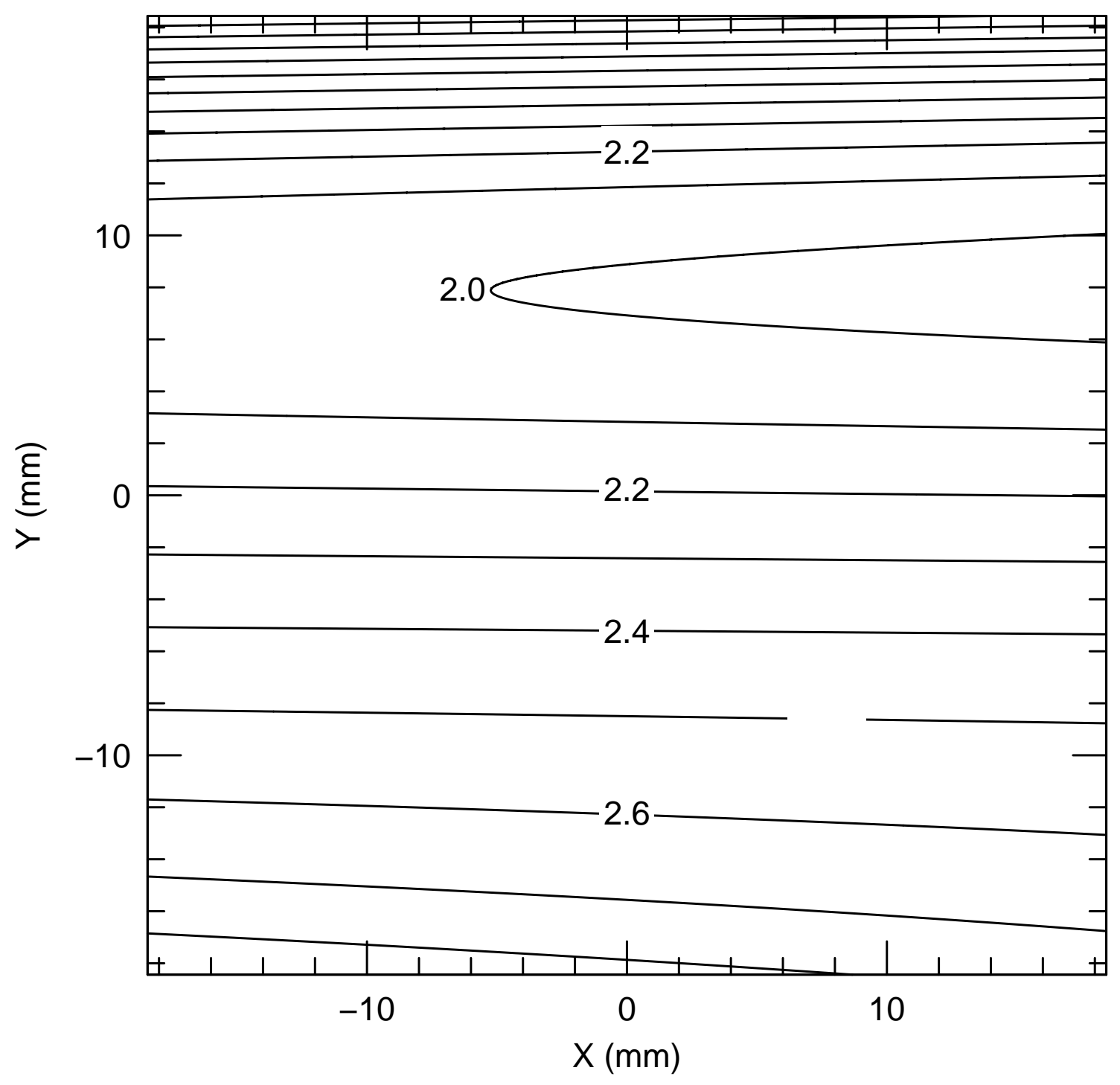

Fig. 3.6. - Contours of constant FWHM for stellar images in a representative $200 \mathrm{~mm}$ lens KELT image, with FWHM given in pixels. Contours are based on a smooth polynomial surface fit to measurements of $\sim 1200$ bright, unsaturated stars distributed across the image. Contour spacing is every 0.1 pixels, with particular contour level values in pixels as indicated. 


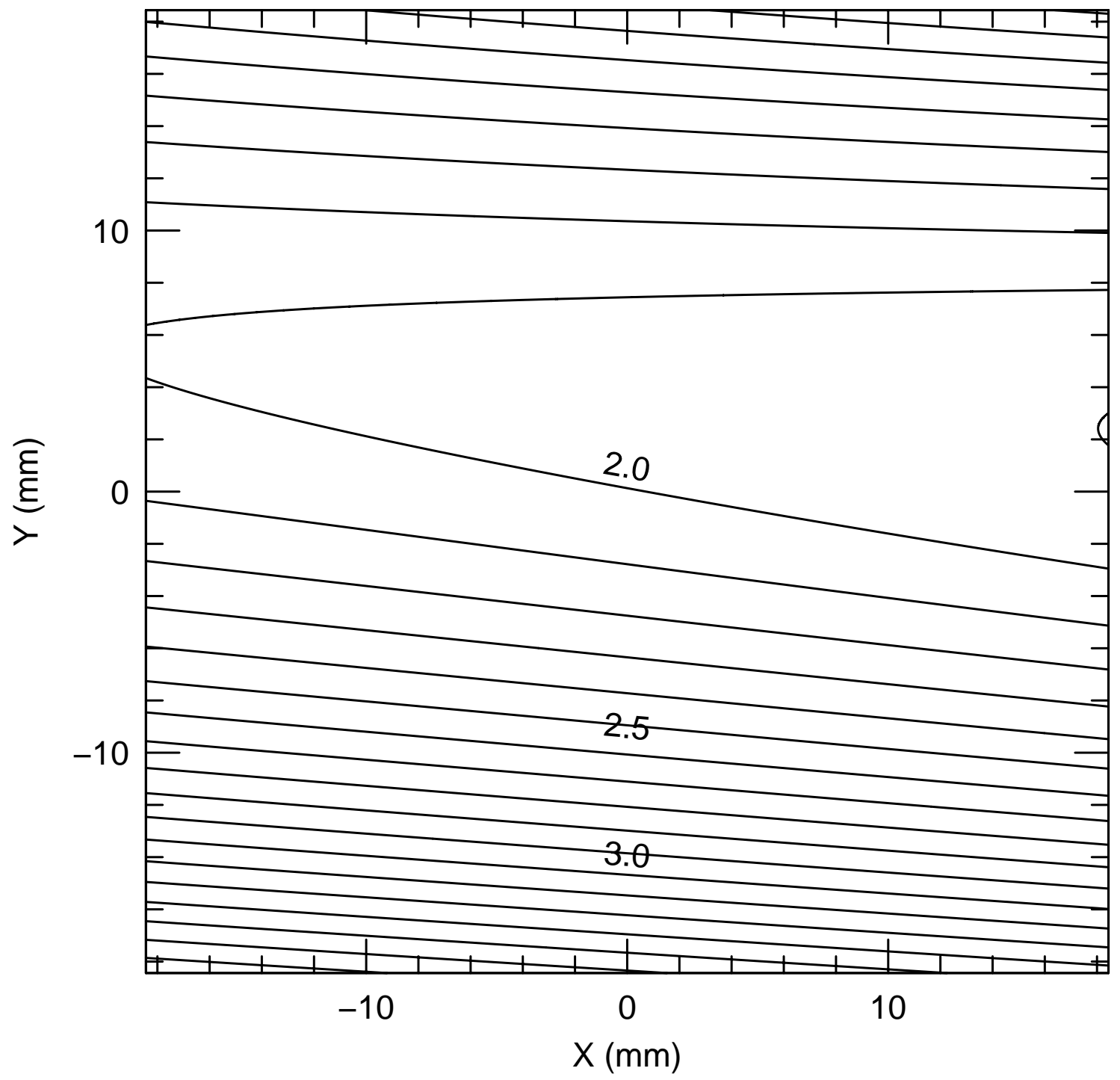

Fig. 3.7.- Contours of constant FWHM for stellar images in a representative $80 \mathrm{~mm}$ lens KELT image, with FWHM given in pixels. Format is the same as in Figure 3.6. Contours are based on measurements of $\sim 2100$ bright, unsaturated stars. 


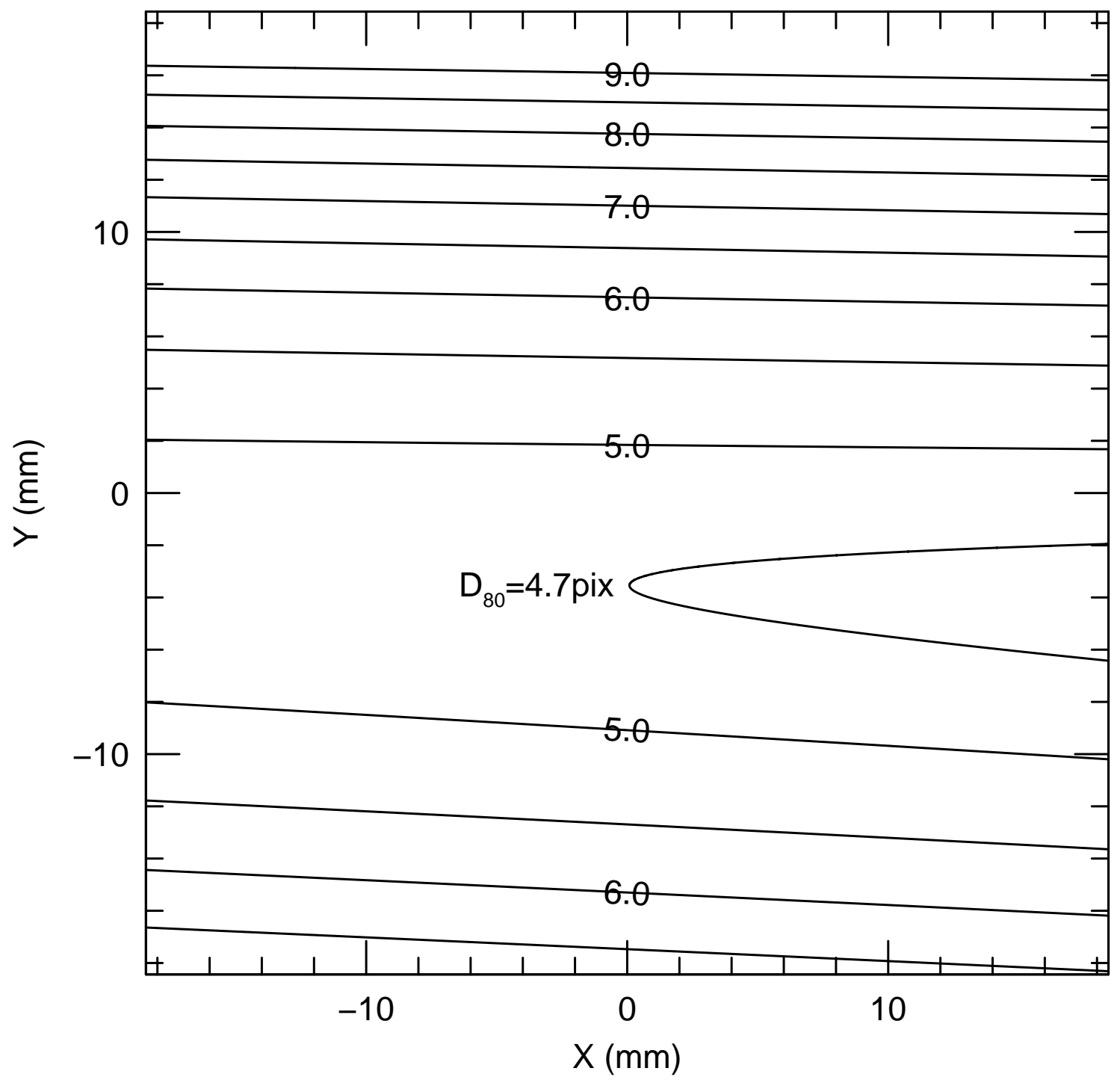

Fig. 3.8. - Contours of constant $80 \%$ Encircled-Energy Diameter $\left(D_{80}\right)$ in pixels for stellar images in a representative $200 \mathrm{~mm}$ lens KELT image. Contours are based on a smooth polynomial surface fit to measurements of $\sim 1200$ bright, unsaturated stars distributed across the image. 


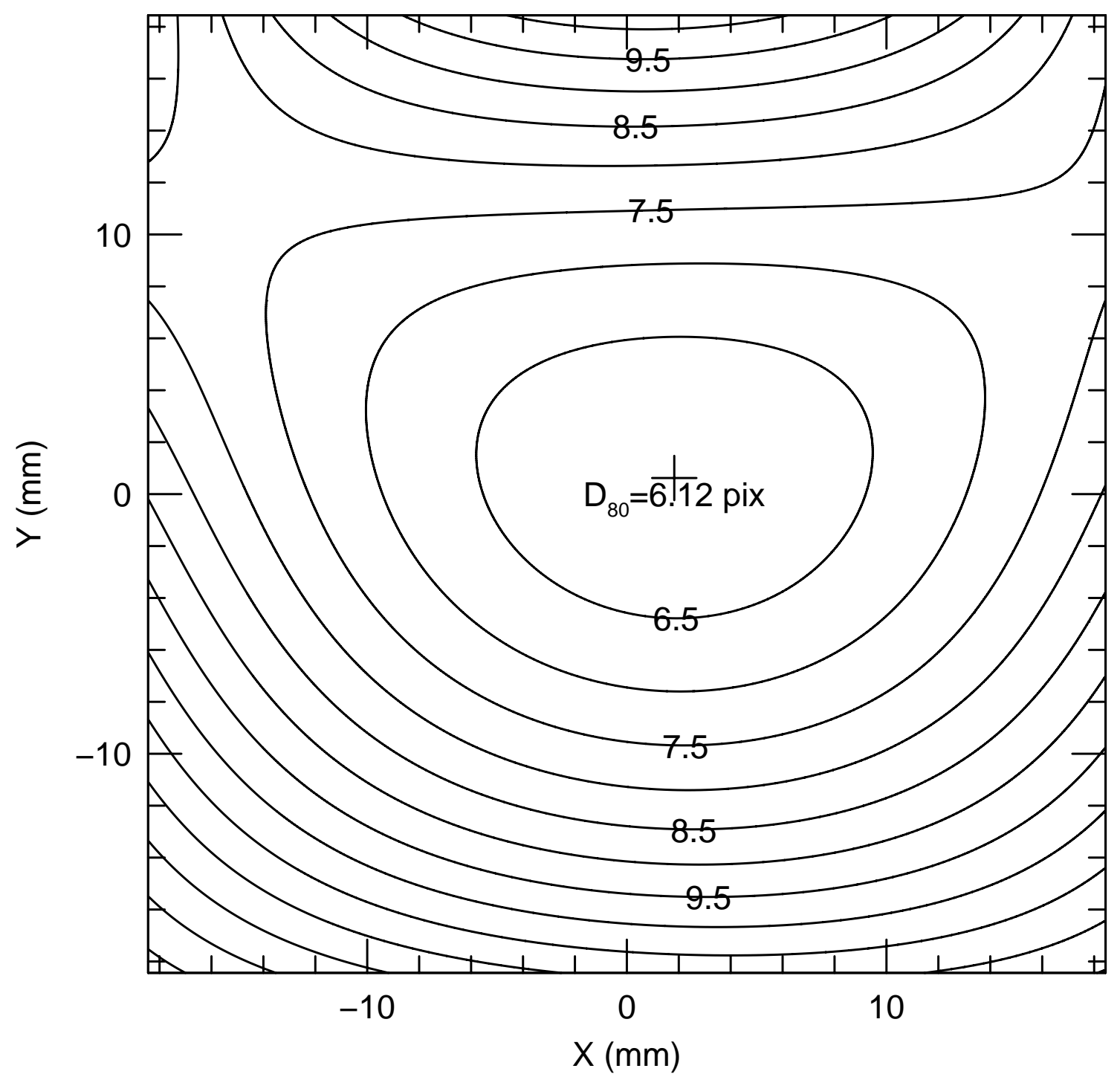

Fig. 3.9.- Contours of constant $80 \%$ Encircled-Energy Diameter $\left(D_{80}\right)$ in pixels for stellar images in a representative $80 \mathrm{~mm}$ lens KELT image. Contours are based on measurements of $\sim 2100$ bright, unsaturated stars. 


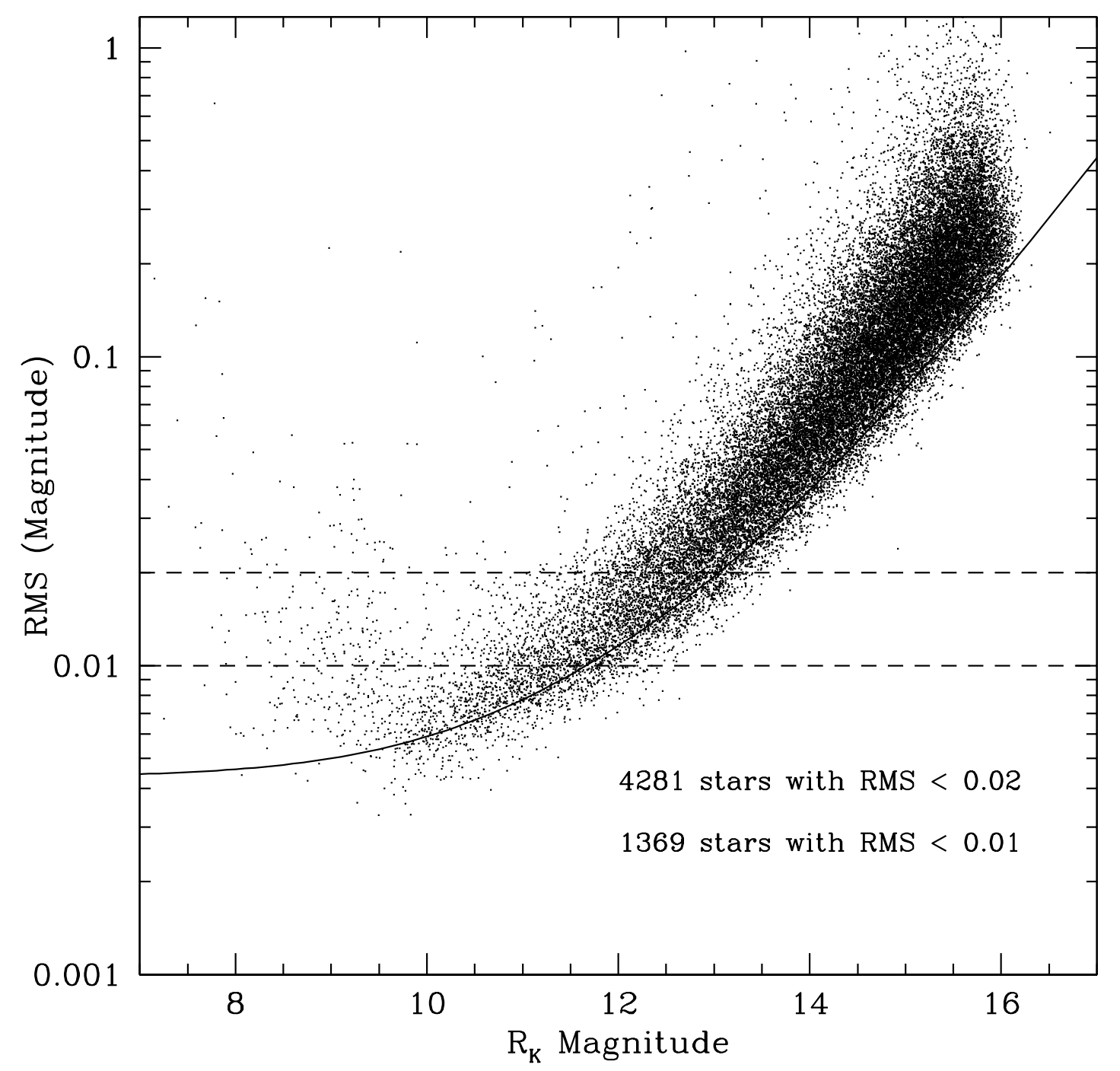

Fig. 3.10.- RMS plot for one night of data from the $200 \mathrm{~mm}$ lens. Data are shown for 67,674 stars. The dashed lines show the limits for $1 \%$ and $2 \%$ RMS. The solid line represents a noise model including photon noise and sky noise, along with an RMS floor of 0.004 magnitudes. 


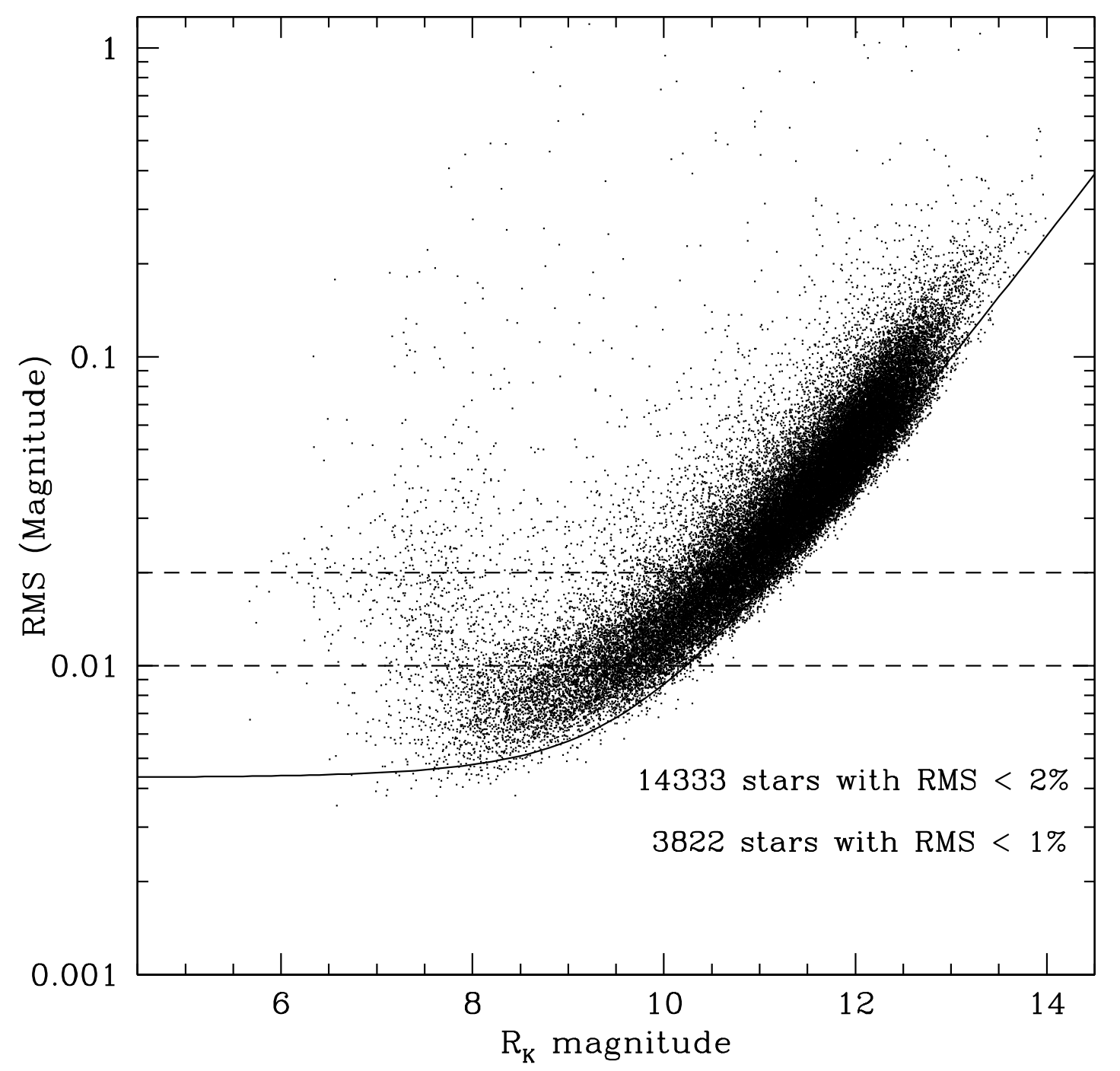

Fig. 3.11. - RMS plot for eight nights of data from the $80 \mathrm{~mm}$ lens. Data are shown for 49,376 stars. The dashed lines show the limits for $1 \%$ and $2 \%$ RMS. The solid line represents a noise model including photon noise and sky noise, along with an RMS floor of 0.004 magnitudes. 


\section{Chapter 4}

\section{KeLt Commissioning Observations: A Photometric Survey for Variables and Transits IN THE Field OF PRAESEPE}

\subsection{INTRODUCTION}

In the previous chapter we described the design, construction, deployment, and observing performance of the KELT telescope. In this chapter, we report the results of our commissioning observations. These data were used to establish our operational procedures and to build and test the data analysis pipeline software. The purpose was not to discover transits with these observations, but rather to use the data to build the analytical tools for use with comprehensive surveys with KELT. The choice to observe the cluster Praesepe was made based on convenience, as it was a large, accessible target observable all night at the time of year of the KELT commissioning runs. Further, Praesepe presented considerable scientific potential for studying the variable star population of the cluster. The details of the data set analyzed here are not identical to the main KELT survey data, but we can use the observations to test our ability to obtain transit-quality photometry, defined as lightcurves with low 
noise, both random and systematic, and able to discern astrophysical signals on the timescale and magnitude of transits.

We first describe the Praesepe observations (§4.2). We then go through the data reduction process, $(\S 4.3)$, with special attention to the problems that affected the data quality (\$4.3.1), and assess the photometric precision of the data set (§4.3.5). We explain how we search for variable stars $(\S 4.4)$ and transit candidates $(\S 4.5)$. We then list the properties of all variable stars detected and display the lightcurves of the periodic variables $(\S 4.6)$, and describe the final set of transit candidates and the observations to confirm their nature $(\S 4.7)$.

\subsection{OBSERVATiOns}

The commissioning campaign targeted a field centered on the open cluster Praesepe (also called M44 and NGC 2632). Known from antiquity as the "Beehive" for its naked-eye appearance on the sky, Praesepe is located in the constellation of Cancer at a distance of 180 parsecs. It has an age of $600 \mathrm{Myr}$, a metallicity slightly above solar, and little or no interstellar extinction (An et al. 2007). Several previous studies have examined the cluster population in efforts to determine the luminosity function of the stars and establish the overall cluster membership (Jones \& Stauffer 1991; Adams et al. 2002), in addition to probing the low-mass end of the stellar population comprising the cluster(Chappelle et al. 2005). According 
to the NASA/IPAC Extragalactic Database ${ }^{1}$ the amount of interstellar reddening $E(B-V)$ towards the center of Praesepe is 0.029 mag (Schlegel, Finkbeiner, \& Davis 1998), corresponding to $A_{V}=0.09$ for $R_{V}=3.1$. This low value is consistent with the cluster's relative proximity to Earth.

The KELT observations were centered on a field located at J2000 $8^{\mathrm{h}} 40^{\mathrm{m}} 06^{\mathrm{s}},+19^{\circ} 41^{\prime} 06^{\prime \prime}$ roughly centered on Praesepe. The observing campaign was carried out every clear or partially clear night from UTC 2005 February 13 through April 27. We obtained 5220 images during 34 out of the 74 nights of the observing run. The observations consisted of repeated 60-second observations throughout the night as long as the cluster was above the horizon, usually taking 100 - 200 images each night. The telescope took images on all nights except those with heavy cloud cover or rain. The quality of our observed nights ranged from completely clear to patchy cloud cover. We rely on several steps of the data reduction process to eliminate images with excessive cloud cover, moonlight, or other problems that compromise photometric quality.

The pointing of the KELT telescope was not perfect, with the field center drifting slowly between images throughout the night. The typical intra-night drift was $\sim 25^{\prime}(\sim 160$ pixels $)$ in Declination and $\sim 9^{\prime}(\sim 60$ pixels $)$ in Right Ascension over the course of many hours. The drift is small compared to the size of the field $(<5 \%)$, but it has two significant effects on our data. First, the drift causes stars

\footnotetext{
${ }^{1}$ http://nedwww.ipac.caltech.edu/forms/calculator.html
} 
at the edges of the field to enter and exit the camera's field of view during the night, resulting in incomplete lightcurves for those stars. However, since the image quality is poor at the extreme edges of the field, we simply eliminate stars along the field edges from our sample. Secondly, the drift combines with our image reduction software to cause constant stars in certain parts of the field to spuriously appear as variable candidates. See sections $\S 4.3 .2$ and $\S 4.4 .2$ for details of this effect.

\subsection{DATA ReDUCTION}

For each of the 5220 images of Praesepe, we subtracted the combined dark for that night, and then divided by the flatfield. We eliminated 2083 poor quality images, defined as images with a sky-level less than 800 ADU or a mean FWHM of the stars in the image greater than six pixels. To obtain photometry, we use the ISIS image subtraction package from Alard \& Lupton (1998); Alard (2000). We follow the implementation of the package as described by Hartman et al. (2004). We describe that procedure below, and we note the change when when our procedure differs from that of Hartman et al. (2004).

\subsubsection{Changing FWHM}

After initial image processing with ISIS, we found that a large number of variable star candidates found during an initial reduction of the data had 
double-valued lightcurves with the amplitude of many sinusoidal lightcurves being larger on some nights and smaller on others. The variation in amplitude was correlated in time. This effect also appeared to apply mostly to stars in a horizontal zone across the upper part of the field. We traced the origin of this effect to changing FWHM of the stellar images across our field from night to night. The FWHM does not change significantly horizontally across the field, but it has significant structure in the Y-direction, with the FWHM being large ( $\sim 2.8$ pixels $)$ at the top and bottom of the field, and decreasing linearly towards the middle of the field, with a minimum FWHM of $\sim 2.0$ pixels at about one third of the distance from the top of the field.

Had the size and shape of this pattern remained constant throughout the observations, it would not have presented a major problem, since ISIS is able to work with a changing FWHM across the field. However, the vertical axis position of the FWHM minimum changed significantly between different nights, ranging from the middle of the field to the top edge, with the position of the minimum correlating with the change in amplitude of the lightcurves. The reason for the double-values lightcurves relates to the way ISIS works. ISIS requires a reference image that has the smallest FWHM of all images in the set, since it convolves the reference image with the kernel of each of the individual images. With the changing shape of the FWHM pattern in our data, no single image or set of images has a smaller FWHM across the entire field for all the nights. Any given choice for a reference image will contain a horizontal region for which there are other nights where the stars have 
smaller FWHM values. Regions with smaller PSFs than the reference image on certain nights require deconvolution of the PSFs (i.e. adjusting the stellar images from the reference frame to have smaller PSFs rather than larger). This creates a problem since ISIS works to convolve an image, even with a varying degree across the field, but ISIS is not equipped to correctly deconvolve an image.

We have not been able to absolutely determine the origin of the time-varying FWHM pattern. We believe it could be related to temperature changes from night to night, which affected the $200 \mathrm{~mm}$ lens we used for these observations. Since the problem was not detected until after we stopped using the lens, we were unable to test this hypothesis. Instead, we intend to mitigate the impact of this effect as much as possible and obtain the best measurements we could out of the data set. The procedure we adopt is as follows. We first register the images to the same coordinate system, then divide all of them into four horizontal sections. For each section we identify a different reference image that has a smaller FWHM than all of the other images in that section. We then treat the different sections as four separate fields, and run each field section though the data reduction pipeline with their own reference images to obtain lightcurves. We also convolve all of the images (but not the reference image) with a Gaussian smoothing function (using a Gaussian with $\sigma=0.6)$ to slightly broaden the PSFs, and thus ensure that the reference image has a smaller FWHM. With this procedure we are able to eliminate most of the effects of the time-varying FWHM. We are not able to completely eliminate the effect, 
which can be seen in the slightly double-valued lightcurves of some variables (e.g., see plots of KP300133 and EF Cnc in Figure 4.8, as well as excessive scatter in the lightcurves of KP113808 and KP118899 4.12).

\subsubsection{Image Subtraction}

We first register all images to a common coordinate system, then divide each image into four horizontal sections, as described in $§ 4.3 .1$. For each section we create a reference image from a combination of the best quality images from that section. We perform image subtraction on each section of the 3137 images with ISIS. We use a feature of ISIS to further subdivide each section into a grid of subfields, each of which can take on different values for the parameters that are used to convolve the reference image with the kernel for image subtraction. This step is particularly advantageous for large fields of view, in which cloud patterns can be smaller than the size of the field. We subdivide each section into grids from $1 \times 5$ to $5 \times 5$, depending on the size of the section, and proceed with the image subtraction.

We use DAOPHOT (Stetson 1987) to identify all of the objects in the field of the reference image of each section, yielding a list of 69,337 stars on all four sections. Stars along the image edges have particularly bad lightcurves, and so we remove all stars within 50 pixels of the edge from further analysis, leaving 66,638 stars for which we generate lightcurves. The ISIS photometry program calculates the flux from a 
star and its error in each image. In some situations, such as when the star is located at the edge of the field, or on the edge on one the of subregions of the subtracted image, the reported flux and error values are not indicative of the true flux. We clean such points from the data by removing data points where the reported flux or error is unphysically high or low. We also remove the two highest and lowest flux measurements from each lightcurve. Removing such a small number of points should not affect detection of variability or transits, but it does help remove spurious points and reduces the number of false positives when searching for variable sources.

\subsubsection{Astrometry And Matching to Known Sources}

We use the Astrometrix ${ }^{2}$ program to derive astrometric solutions for the reference image, using the Tycho-2 catalog (Høg et al. 2000) to select reference stars. Because of high-order distortions in the corners of the field, we first subdivide the full images into 25 subsections and find separate astrometric solutions for each subimage. The astrometry is good to within an arcsecond, or about $10 \%$ of a pixel.

We match our data set to two catalogs. We first match our stars to the 2MASS catalog (Skrutskie et al. 2006), using a search radius of 9.5 arcseconds or about one KELT pixel. We find that 58,620 out of 66,638 of our KELT stars match to a 2MASS source, with 1,559 of them matching to more than one $2 \mathrm{MASS}$ source. The

\footnotetext{
${ }^{2}$ http://www.na.astro.it/ radovich/wifix.htm
} 
fact that a small portion of the KELT stars match to multiple 2MASS sources is to be expected, since the 2MASS catalog goes much deeper in magnitude than KELT and so identifies a greater density of sources on the sky. In such a case we would expect that a small fraction of KELT stars would randomly match to more than one 2MASS source, especially since we use a search radius larger then the astrometric resolution of 2MASS.

We also match our star catalog to known members of the Praesepe cluster. We compiled a catalog from the WebDA website,$\sqrt[3]{3}$ identifying 832 likely cluster members. After matching to the KELT data using a search radius of 9.5 arcseconds, we find matches to 333 Praesepe member stars. However, many of the stars in the WebDA database are too faint for KELT to detect. If we consider only the 210 WebDA sources with known $V$ magnitudes of $6.8<V<16.4$, we find matches to 147 stars, although the brighter stars are mostly saturated and unusable in the KELT images.

\subsubsection{Rescaling ERRORS}

One feature of ISIS that has been noted by others is that the formal reported errors tend to be underestimated for brighter stars, such that the reduced $\chi^{2}$ is much larger than unity. Since the errors on individual points are important in the variable

\footnotetext{
${ }^{3}$ http://www.univie.ac.at/webda/webda.html
} 
selection process, we rescale the errors following the procedure of Kaluzny et al. (1998) and Hartman et al. (2004).

We first compute the reduced $\chi^{2}$ for every star. We then plot $\chi^{2} / N_{\text {dof }}$ versus magnitude and fit a curve to the bottom edge of the heaviest concentration of points. We then multiply the formal errors by the square root of the function that curve, so that the least variable lightcurves in our data set have $\chi^{2} / N_{\text {dof }}$ close to 1 for all magnitudes.

\subsubsection{Photometric Precision}

A common method for describing the photometric precision of transit searches is to plot the magnitude root-mean-squared (RMS) of all the lightcurves as a function of magnitude. Because of the FWHM changes described in $\S 4.3 .1$, the long term photometric precision has been degraded. However, since the intra-night FWHM pattern appears to be stable, we plot both for all 66,638 stars the overall RMS and the RMS for one of the nights in Figure 4.1. Panel (a) of Figure 4.1 shows the RMS plot for a single night of data, while panel (b) shows the RMS plot for the full 34 nights of data. The dashed horizontal lines show the $2 \%$ and $1 \%$ RMS limits, which generally define the required sensitivity for detecting Hot Jupiter transits. Two features stand out in these plots. The first is that there are significantly fewer stars with RMS $<1 \%$ in plot (b) than plot (a), although there are about the 
same number of stars in each plot with RMS $<2 \%$. This behavior shows that the inter-night systematics, of which we believe the FWHM changes to be the most significant, become most important at the sub- $1 \%$ level. The second feature to note is the greater RMS of stars brighter than $R_{K} \approx 9$. The lightcurves of the brightest stars in our sample are dominated by systematic saturation and/or nonlinearity effects that are present during a single night but are more severe over the entire run.

\subsection{VARiable Selection}

Any transit search will yield a data set ideal for detecting variable stars that are unrelated to transiting planets. We implement several cuts to select promising variable star candidates. We first employ the Stetson J statistic (Stetson 1996) to find sources that vary coherently in time. We then remove long period variables (LPVs) and run a period-search algorithm to find periodic variables based on the $\sigma_{A o V}$ statistic. We run a periodogram filter to remove spurious variables due to aliasing, resulting in a final set of variable star candidates. Finally, we visually inspect the remaining lightcurves to remove false positive.

\subsubsection{Stetson Statistic}

For each of the 66,638 stars, we compute the Stetson $J$ statistic, using the implementation from Kaluzny et al. (1998). This statistic identifies coherent variable 
stars by selecting for photometric variations that are correlated in time. The statistic is defined by

$$
J=\frac{\sum_{k=1}^{N} w_{k} \operatorname{sgn}\left(P_{k}\right) \sqrt{\left|P_{k}\right|}}{\sum_{k=1}^{n} w_{k}}
$$

where the observer defines $N$ pairs of observations with weights $w_{k}$. We define observations as a pair when they are separated by less than 10 minutes. $P_{k}$ is the product of the normalized residuals of two observations $i$ and $j$, constituting the $k$ th pair, such that

$$
P_{k}=\left\{\begin{array}{cc}
\delta_{i(k)} \delta_{j(k)}, & \text { if } i(k) \neq j(k) \\
\delta_{i(k)}^{2}-1, & \text { if } i(k)=j(k)
\end{array}\right.
$$

and $\delta$ is the "relative error", defined as

$$
\delta=\sqrt{\frac{n}{n-1}} \frac{m-\mu}{\sigma},
$$

where $m$ is the apparent magnitude of the star, $\sigma$ is the error in the magnitude, and $\mu$ is the weighted mean magnitude of the star. We choose weights of $w_{k}=1$ for pairs of observations $(i(k) \neq j(k))$ and $w_{k}=0.25$ for single observations $(i(k)=j(k))$, and we also multiply the final quality $J$ by $\sum_{k=1}^{n} w_{k} / w_{\max }$, where $w_{\max }$ is the total weight the star would have if it were measured on all images.

After visually examining a number of lightcurves, we define a cutoff of $J=0.7$ to select variable candidates, deliberately choosing a liberal cut on $J$ since we 
have several more tests to filter out non-variables. We eliminate those stars with $R_{K}<9$, since the lightcurves of the brightest stars in our data set are dominated by systematics due to saturation effects. We also eliminate any star that is less than 10 pixels away from stars with $R_{K}<9$, since extended wings and bleed trails from the bright stars create false variability. We finally remove any candidate that is less than 13 pixels away from a variable candidate with a higher RMS in flux. This eliminates false positives due to constant stars close to true variables. After these cuts we are left with 3,430 candidate variable stars.

\subsubsection{Variable Clustering}

Among the 3,430 variable candidates that pass the cuts described above, most of the stars are clustered around a few of the subsection boundaries, mostly between subsections along the field edges where the fitting parameters vary most, and adjacent subsections closer to the field interior. We assume that all spatial clustering of variables is due to artifacts in the reduction process, and we suspect that the reason for the clustering has to do with how large changes in PSF size and shape across the field affect ISIS.

As described in $\S 4.2$, there was an intra-night drift in the telescope pointing. One effect of this drift is that stars undergo slight changes in the PSF shape and size during each night. When ISIS convolves the reference image, it uses different 
parameters for each subsection (see §4.3.2). Subsections at the edges of the field experience the strongest optical distortions due to the wide field, and ISIS has the most difficulty fitting the convolution parameters in those areas. At the edges of those subsections the assumptions used to compute the fitting parameters break down. For stars in those areas, the intra-night drift means that any consistently inadequate convolution will show up as photometric variability on timescales comparable to the drift rate.

In order to eliminate false variability due to this effect, we want to remove any candidates that appear in areas with high spatial clustering. However, these areas are not precisely defined - they result from the combination of the ISIS subsection grid, the direction and speed of the drift, and the nature of the optical distortions. We therefore devise an algorithm to remove from our list any stars that are in clustered areas. We divide the entire field into boxes 100 pixels on a side, and count the number of variable candidates in each box. We perform this process four times, with each grid offset from the previous one by 20 pixels in both the $\mathrm{X}$ and $\mathrm{Y}$ directions. We thus have, yielding four staggered grids with which to measure the clustering of the variable candidates. We classify all candidates that appear in a box with more than two other candidates in any of the grids as spurious and eliminate them from our sample, leaving 1101 variable star candidates. 


\subsubsection{IDENTIFICATION OF LONG-PERIOD VARIABLES}

There are some stars that pass the cut on $J$ that do not exhibit periodic variability. Many are long-period variables (LPVs) that show monotonically increasing or decreasing brightness during our campaign, some of which may vary periodically but on time scales longer than our campaign. We identify such objects and remove them from our later analysis, which focuses on identifying periodic variables.

To identify LPVs, we use the method described in section 4.3 of Hartman et al. (2004), in which a star is defined as an LPV when a parabola fits the lightcurve much better than a horizontal line. We fit a parabola to the 1,101 remaining variable candidates and calculate the $\chi^{2} / N_{\text {dof }}$ for the fit, which we call $\chi_{N-3}^{2}$, along with the $\chi^{2} / N_{\text {dof }}$ for the fit to the mean, $\chi_{N-1}^{2}$. There are 52 stars for which $\chi_{N-3}^{2} / \chi_{N-1}^{2}>0.8$, and therefore identified as candidate LPVs, which we eliminate from our remaining variable candidate list. We show the lightcurves of three of our LPVs in Figure 4.2.

A much smaller fraction of our stars are LPVs (52 out of 66,638 stars, or $0.078 \%$ ), than the 1,535 LPVs out of 98,000 stars, or $1.6 \%$, found by Hartman et al. (2004). We attribute this difference partly to our greater observational time baseline (74 nights vs. 30 nights), since we end up classifying stars with periodic behavior in that range as regular periodic variables rather than as LPVs. Also, the Praesepe 
field is located well out of the Galactic Plane, and we therefore expect many fewer background giants than in the field that was observed by Hartman et al. (2004).

\subsubsection{PERIODICITY SEARCH}

We use the period search algorithm of Schwarzenberg-Czerny (1996) to select periodic variables, adopting the implementation by J. Devor. The SchwarzenbergCzerny algorithm reports a periodicity likelihood statistic $A o V$, and the Devor method analyses that information to estimate $\sigma_{\mathrm{AoV}}$, a measure of the confidence of the lightcurve's periodicity. We apply the algorithm to the 1,049 stars remaining in our catalog after removal of the LPVs.

The plot of the best-fit period $P$ vs. $\sigma_{A o V}$ (Figure 4.3) shows significant aliasing effects at periods that are integer multiples or fractions of 1 day. We thus need to make further cuts to select the true variables from among our list of candidates.

First, we construct three histograms in $\log (P)$. Each histogram is shifted in $\log (P)$ by $1 / 3$ of the width of a bin. All objects that appear in bins in any of the three histograms that have more than seven objects in them are rejected. Any remaining objects with $\sigma_{A o V}>3.3$ are retained. To ensure that true variables were not accidentally eliminated by the binning procedure, any stars that have $\sigma_{A o V}>4.0$ are automatically retained, even if they are caught by the aliasing identification algorithm. 
After these cuts, we are left with 182 variable star candidates. We then examine the lightcurves of the remaining candidates by hand, and find that 70 of these are false variables, all of which slightly missed the clustering or alias filters. We also find that six of the candidates, all of which have estimated periods longer than 20 days, are in fact LPVs.

\subsection{Transit SEARCH}

The primary goal of the main KELT survey is to identify possible planetary transits. We have used the commissioning data set to build our software pipeline and test our data reduction and analysis procedures, without expecting to identify transit candidates in this data set. However, it is still useful to search for transits, since there is still a chance of discovery, and the transit search could also yield interesting variable stars not found through the earlier variable selection method. Furthermore, we would expect to find false positives in the transit search - astrophysical events that look like transits, such as low-mass stars transiting a solar-type star, which would have lightcurves similar to planetary transits. Finding these events would demonstrate our ability to detect actual transits in data acquired using KELT.

In order to search the data for transits, we take two main steps. The first is to apply a detrending algorithm to reduce or remove a variety of systematics. The 
second is to run a transit search algorithm over the detrended data and identify transit candidates.

\subsubsection{DETRENDING}

Wide-field transit surveys are susceptible to a number of systematic errors. Observing objects for long stretches of the night requires the telescope to cycle through significant changes in airmass. A wide field of view allows differential cloud patterns to complicate the process of obtaining accurate relative photometry. Temperature changes can affect the optics or detector performance. With so many sources of systematic error, it can be prohibitive to attempt to identify, measure, and compensate for all of these effects. Instead, several methods have been developed to identify all generic systematic effects in data sets of this type. The method we choose to implement is the SYSREM algorithm of Tamuz, Mazeh, \& Zuker (2005) (see also Mazeh, Tamuz, \& Zucker 2006). In brief, this algorithm identifies and subtracts out linear trends that appear in a large portion of lightcurves in a given data set.

We apply SYSREM to the KELT lightcurves that have been reduced using the procedures described in $\S 4.3$. Since lightcurves with very large variations can create problems for detrending programs, and are not useful for the identification of low

level systematics, we apply SYSREM to only the 15,012 stars from our data set with RMS $<5 \%$. We recalculate the RMS for the detrended lightcurves and display the 
results in Figure 4.4. For most of the lightcurves, detrending improves the RMS by about $10 \%$, although for a few stars, especially towards the bright end, the RMS improves by a factor of 3 to 4 .

We suspect that the reason that detrending does not improve the RMS to a greater degree is related the the FWHM problems described in $§ 4.3 .1$. Algorithms like SYSREM are designed to remove linear systematics, but it is probable that the systematic errors caused by the FWHM changes are higher order, and so are not rectifiable by linear detrending. Even so, detrending manages to improve the RMS by a small amount, and in some cases works very well, as shown in Figure 4.5. For the periodic variables we identify with non-detrended RMS below 5\%, detrending improves $\sigma_{A o V}$ on average by $10 \%$.

\subsubsection{Transit SElection}

To detect transits, we apply a version of the Box-Fitting Least-squares (BLS) transit-search algorithm (Kovacs, Zucker, \& Mazeh 2002) implemented Burke et al. (2006). This algorithm cycles through a range of periods and phases searching for a transit-like event and selects the one with the highest significance. For each lightcurve, we calculate the $\Delta \chi^{2}$ between a constant flux and the best-fit transit model, the $\Delta \chi_{-}^{2}$ for the best-fit antitransit (a brightening rather than dimming), the fraction of $\Delta \chi^{2}$ that results from a single night $f$, and the transit period $P_{t}$. We 
then examine five parameters: $\Delta \chi^{2}, \Delta \chi^{2} / \Delta \chi_{-}^{2}, f, P_{t}$, and the transit depth. We also examine Digital Sky Survey (DSS) images of the stars which have much higher resolution than KELT, to check whether the single KELT sources consist of blends of more than one star.

Because of the unique characteristics of this data set, we have not elected to construct a rigorous transit selection algorithm using cuts. Instead we sort the lightcurves based on each of the five parameters described above, and examine by eye the 100 best lightcurves identified by each criterion. We classify the lightcurves as either possible transits, variables, or neither. We find four stars with transit-like behavior that are unblended and that are identified with known 2MASS stars. We also find 38 additional variable stars that were not found with the steps described in \$4.4. Of those objects, 31 had $J<0.7$, which is not surprising since the transit-like signals that BLS searches for would not necessarily show the coherent variations characteristic of a variable star with a large $J$ value. Of the seven other stars, one was eliminated by the cluster-rejection routine, and the remaining six had $\sigma_{A o V}<3.3$. 


\subsection{VARIABLE StARS}

After these procedures we are left with 208 variables, which include the 52 LPVs identified in $§ 4.4 .3$ and the 6 LPVs found in $§ 4.4 .4$, the 112 periodic variables found in $\S 4.4 .4$, and the 38 periodic variables found in $\S 4.5 .2$.

\subsubsection{Matching to Known Variables}

According to The General Catalog of Variable Stars (GCVS4.2; Samus \& Durlevich 2004) and the New Catalog of Suspected Variable Stars (NSV; Kukarkin et al. 1982), there are 168 known variables within our field of view. The magnitudes of the variables are reported in either Johnson $V$ or $U$, or photographic magnitude $p$. The range of magnitudes for which we found variables in the KELT data using all the methods described above are $9<R_{K}<16$. Out of the 168 variables, 72 have reported magnitudes outside that range, and an additional 17 are within $15<V<16$. We only try to match known variables with reported magnitudes between 9 and 15 .

We check whether we detect the remaining 79 variables in our data. We searched for KELT counterparts using a matching radius of two pixels $\left(19^{\prime \prime}\right)$ and identified 63 matches. Of the 16 known variables we do not detect, three appear either saturated or blended with saturated stars in our data, and 13 do not appear 
to have counterparts within two pixels of the reported positions, suggesting that the reported positions, proper motions, or magnitudes may be incorrect.

We then compare our list of 208 variables with the 63 detected known variables and find 14 matches. Of the 49 known variables we do not classify as variable through our tests, five have counterparts that are either brighter than $R_{K}=9$ or blended with a bright star. Another 38 are irregular, eruptive, or unspecified variables, which we would not expect to detect with our periodicity-based search algorithms. Three are eclipsing variables of unspecified type with no periods listed in the catalogs. Upon investigation of their KELT counterparts, no eclipses are seen, indicating that either our observations missed the eclipses, or that the eclipse depths were too small to appear in our data. One is listed as an RR Lyrae variable with no period given, for which the KELT lightcurve shows no periodic variation. The remaining two are eclipsing binaries which are removed in the clustering filter, but can be seen in the KELT lightcurves. We add those two stars to our list of variables.

We thus have KELT lightcurves for 16 known variables. We find that one KELT LPV is the semi-regular variable GV Cancri. The lightcurve for this object is shown in Figure 4.2. We find three RR Lyrae variables, CQ Cancri, AN Cancri, and EZ Cancri, the first two of which have reported periods that we confirm. One more variable, EF Cancri, is listed as a W UMa contact eclipsing binary, but the lightcurve, despite the low-quality photometry, appears to indicate that it, too, is an RR Lyrae variable. 
We detect six known eclipsing binaries: EH Cancri, GW Cancri, FF Cancri, RU Cancri, NSV 04207, and NSV 04158, in addition to two eclipsing binaries that were caught by the clustering filter: TX Cancri and RY Cancri. We confirm the reported periods of RY Cancri and TX Cancri, but we find that RU Cancri has a period of 10.0591 days rather than the reported period of 10.172988 days. The remaining variable stars do not have previously reported periods.

The star NSV 04269 is listed as a semiregular variable in the NSV catalog, but our lightcurve shows it to be an eclipsing binary. The star NSV 04069 is not listed as any variable type; we classify it as an eclipsing binary. Lastly, the star FR Cancri is listed as a BY Draconis variable, consistent with our lightcurve.

\subsubsection{Variable Star Catalog}

We list the properties of our variable stars in Tables 4.1 and 4.2 . For each star we give the KELT ID number, mean $R_{K}$ magnitude, coordinates in Right Ascension and Declination (J2000.0), JHK colors from 2MASS and the 2MASS ID for those that matched to 2MASS objects, period (for non-LPVs) in days, type of variable based on our classification, and the GCVS or NSV ID and classification for those that we have identified with previously known variables.

We plot the 2MASS infrared color-magnitude diagram (CMD) for all our stars in Figures 4.6 and 4.7. In Figure 4.6 we show Praesepe cluster members along with 
all background stars. The cluster members form a coherent main sequence. The background stars form three populations in $J-K$ color. The largest group, at $J-K \sim 0.4$ consists of main sequence stars. The group at $J-K \sim 0.6$ consists of red giants, and the last group, at $J-K \sim 0.8-0.9$, consists of nearby late-type stars, clearly overlapping the same stellar population at the low end of the Praesepe cluster.

We plot the lightcurves of the periodic variables we identify with the methods described above, along with the two previously known periodic variables that missed our cuts. We classify 48 variable as pulsators, and we plot their lightcurves in Figures 4.8 and 4.11. We classify 105 variables as eclipsing binaries, and we plot their light curves in Figures 4.12 through 4.20 .

\subsection{Transit Candidates}

Using the selection process described in $\$ 4.5 .2$, we identify four possible planetary transit candidates. The lightcurves of the four candidates are shown in Figure 4.21. These four lightcurves all show events with depths of $\lesssim 5 \%$, periods between 0.5 and 2 days, and that are not blended with nearby stars in DSS images.

In order to determine whether any of these candidates are planetary in nature, it is necessary to check whether the signals we detected are false positives. There are a number of configurations that can cause transit-like lightcurves through 
non-planetary means (Brown 2003). Since M-dwarf stars are about the same size of Jupiter-mass planets, an M star orbiting an F or G star will cause an eclipse with the same depth as a planetary transit. If a normal eclipsing binary star is blended with a foreground or background star in the survey images, the resulting combined lightcurve would show the eclipses of the binary diluted to the level of a planetary transit dip. Another common signal is a grazing eclipsing binary, where the secondary star passes just along the edge of the disk of the primary star causing a small dip, although in such cases the shape of the dip will be more V-shaped than that of a planetary transit.

To rule out any of these common false positives, we performed low-resolution spectroscopy on each of the four candidates. By taking a several spectra of each candidate, we can quickly distinguish whether any of the three false positive described above are the cause of the signal, and we can therefore rule out the cause of the signal as planetary. The candidates were observed during January and March of 2007 using the CfA Digital Speedometer on the 1.5 meter Tillinghast Reflector at the F. L. Whipple Observatory at Mount Hopkins, Arizona.

The combined photometric and spectroscopic observations show that three of the four candidates are astrophysical false positives. 
- The spectrum of KP20094 is double-lined and shows rapid rotation - clear signals of a binary star. Combined with the V-shape of the dip, the data shows this candidate to be a grazing eclipsing binary.

- The lightcurve of KP102662 is problematic. The curve has greater scatter than the others, and the dip is fairly deep - about $4 \%$ - to be of planetary origin. The spectroscopic observations show low rotation and low RV residuals, but the V-shape of the dip is suggestive of a diluted binary

- KP102791 has a promising lightcurve. The BLS-reported period is 1.5107 days, with a flat-bottomed dip of about 1.5\%. Spectroscopic observations, however, find that it is an eclipsing binary, with an early $\mathrm{F}$ star primary with $T=7000 K$. The actual period is 3.02267 days, which we can determine through the RV observations. The primary eclipse was not caught in the KELT observations, which resulted in the incorrect period determination.

- The nature of KP103126 remains undetermined. The lightcurve shows a small but detectable dip with small scatter. However, the signal is very small and the period is short - about half a day, suspiciously close to an aliasing period. Spectroscopic observations indicate that the star is a late F or early G dwarf, and show slow rotation and no significant velocity variations. This remains a possible planetary transit candidate. 
The only remaining viable planetary transit candidate is KP103126. The next step in determining its nature is to obtain higher-precision follow-up photometry. Normally, that would be done by determining the ephemeris for an upcoming transit and scheduling observations to catch the entire transit from ingress through egress. Unfortunately, the amount of time that has passed since the original KELT observations, combined with their 74-night observational baseline and the short period have made it impossible to accurately calculate an ephemeris to better than six hours. Instead, we plan to follow this candidate up in the future with small-telescope photometry to determine the transit time and period. 


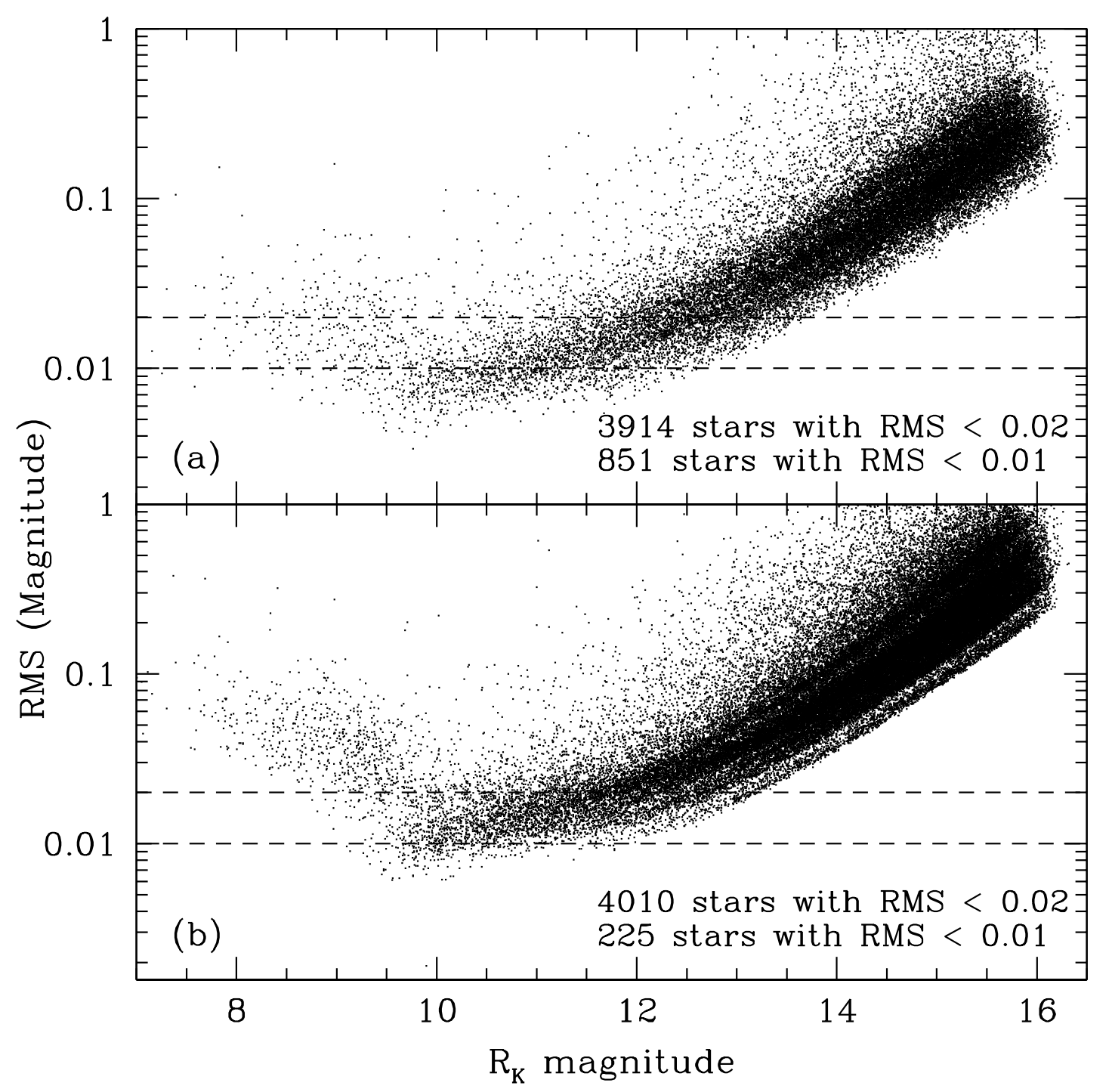

Fig. 4.1.- (a) RMS vs. $R_{K}$ magnitude for the 66,638 KELT stars, calculated from 105 observations over the course of one night. (b) RMS plot for the same stars using all 3,137 observations over the full 34 nights. Structure in the plots results from the separate reductions of the four sections of the field to deal with the changes in the FWHM pattern. 


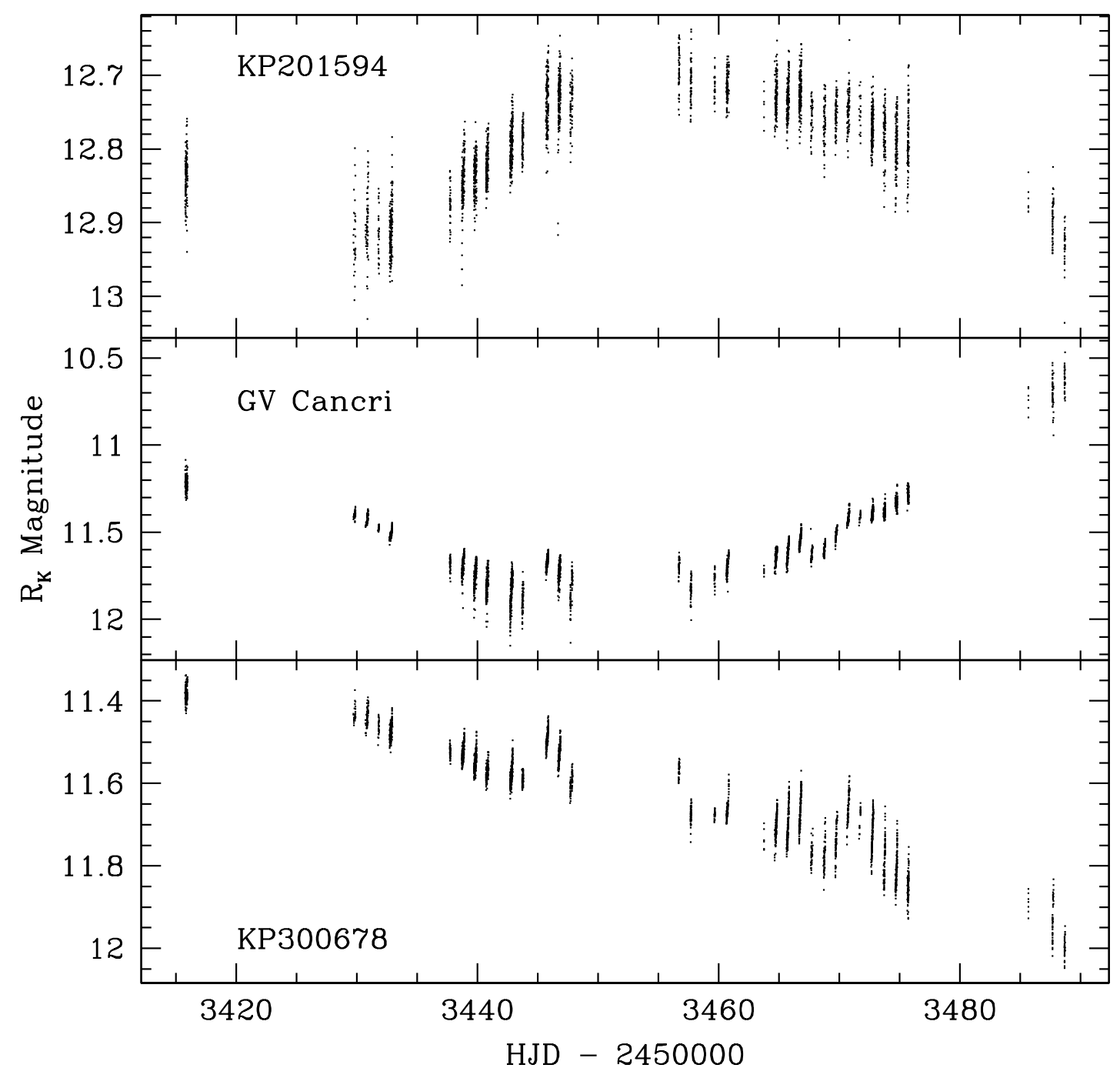

Fig. 4.2. - Lightcurves of three LPVs. We identify KP300603 as semiregular variable star GV Cnc. 


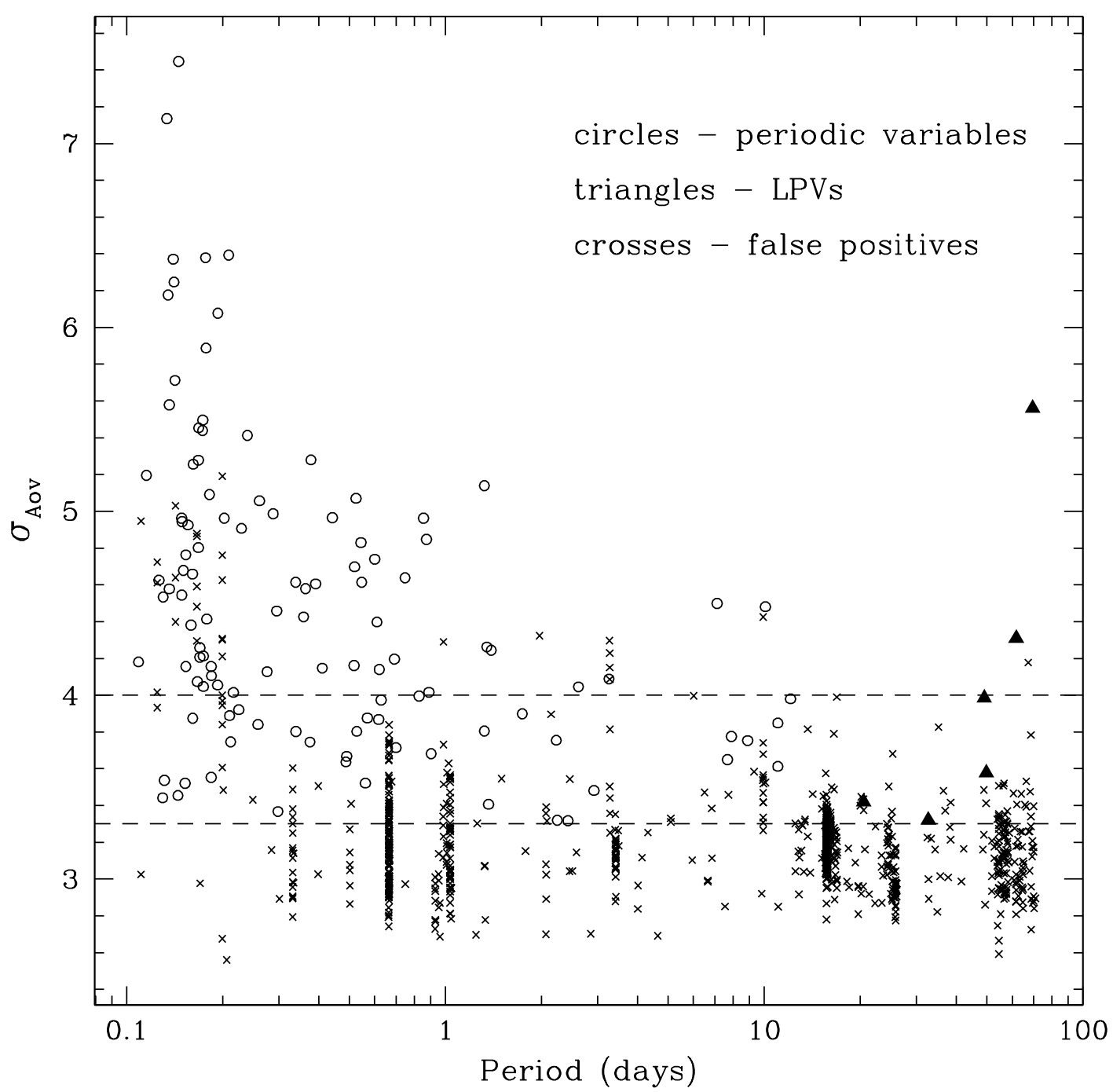

Fig. 4.3.- Plot of period vs. $\sigma_{A o V}$ for the 1,049 stars left after initial removal of LPVs. The dashed lines show the two cuts on $\sigma_{A o V}$. Stars classified as periodic variables are marked by circles and false positives are marked by crosses. As small number of stars that were not removed by the automatic filter for LPVs are determined upon visual inspection to be LPVs, and are marked by triangles. 


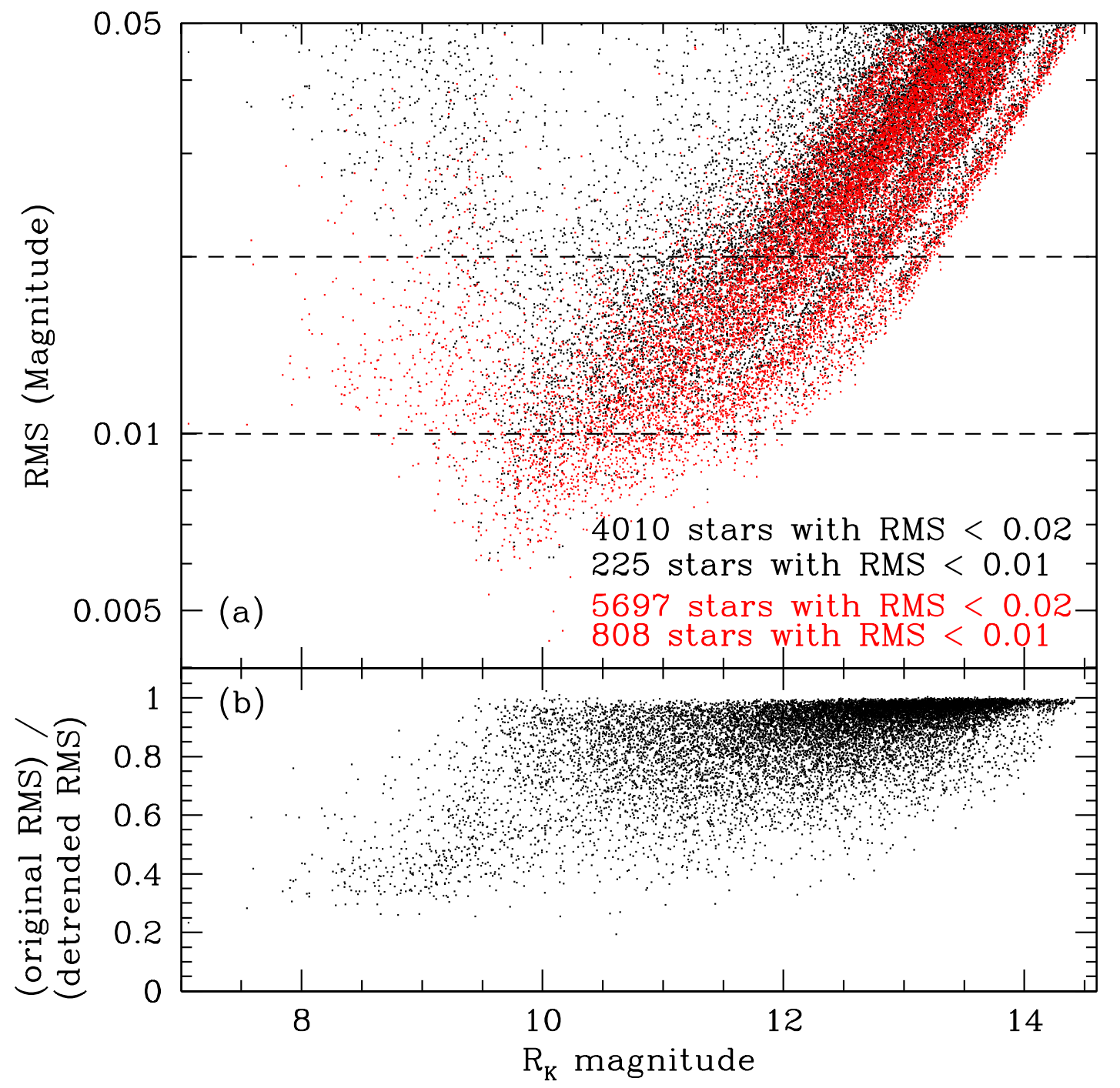

Fig. 4.4.- Panel (a) shows the RMS plot for 15,012 stars before (black) and after (red) application of SYSREM detrending. Structure in the locations of the fainter stars results from the separate reductions of the four regions to compensate for the changes in the FWHM pattern. Panel (b) shows the change in RMS by magnitude. 


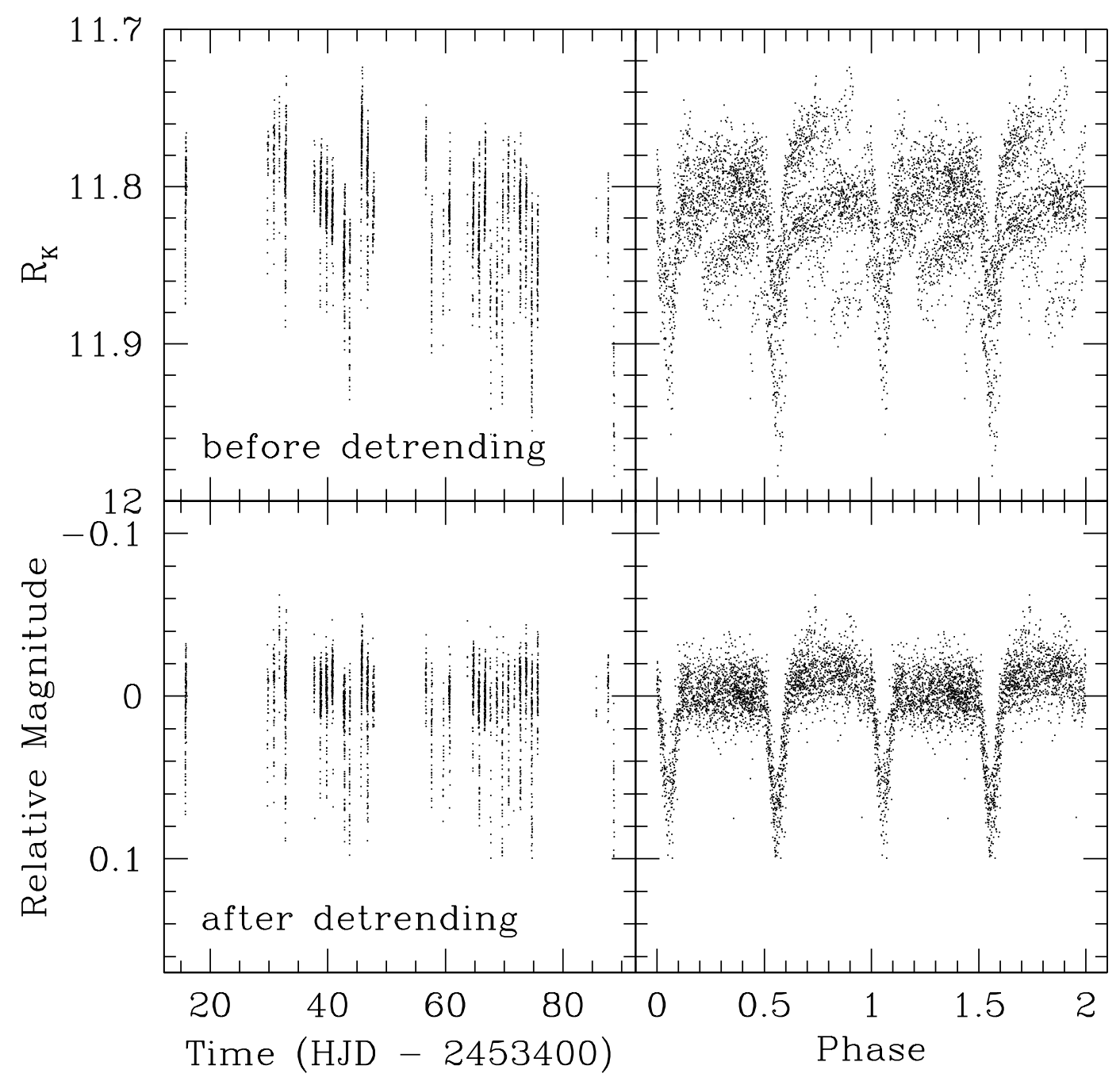

Fig. 4.5. - Lightcurves of the detached eclipsing binary KP102511 (Period=0.5584 days) before and after detrending with SYSREM. The value of $\sigma_{A o V}$ increased from 3.1 to 4.8 . 


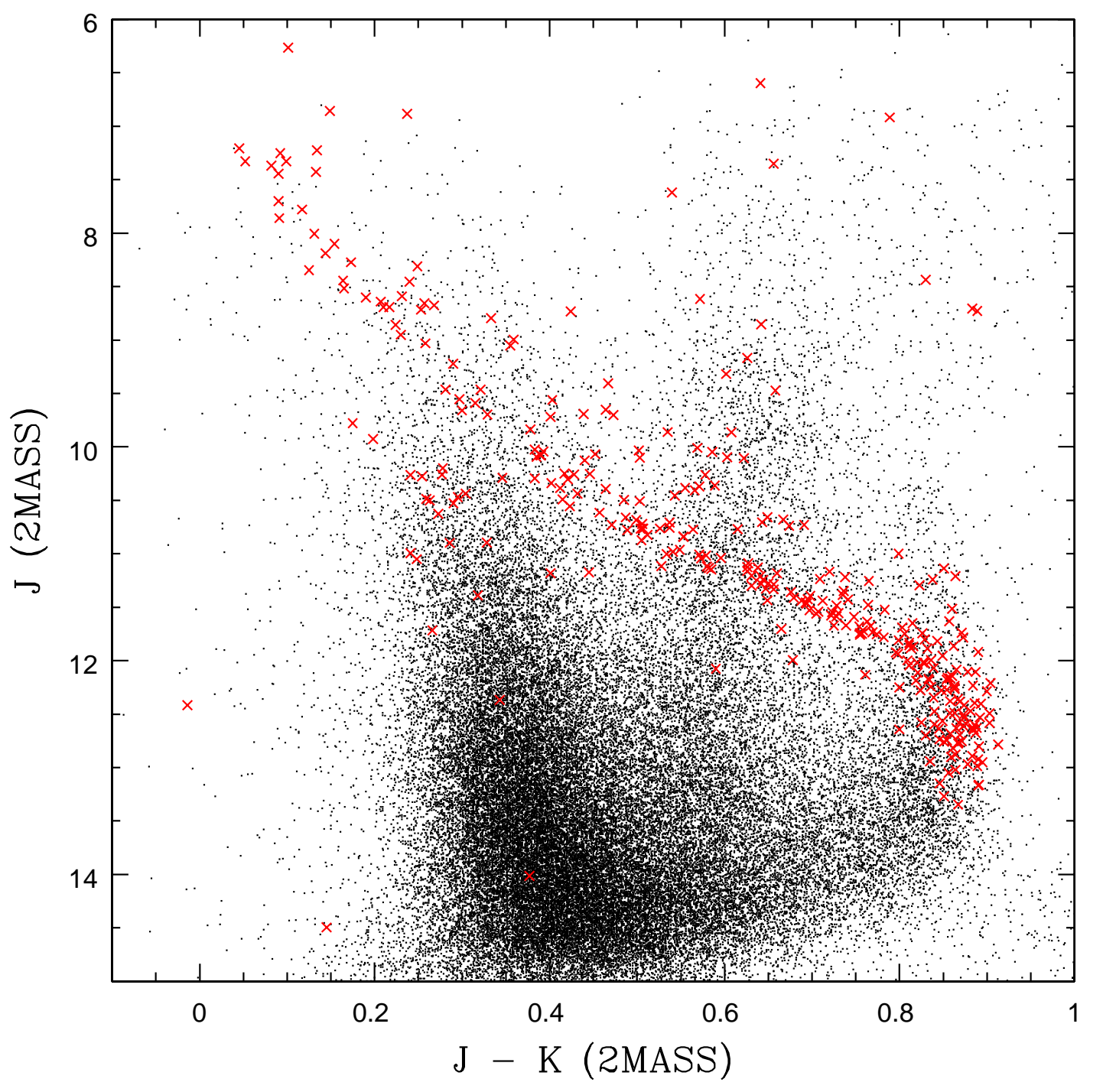

Fig. 4.6. - Color-magnitude diagram for cluster stars and field stars. Only field stars that match to a single 2MASS source are plotted; all cluster stars have unique 2MASS matches. Red crosses mark stars listed as members of Praesepe by WedbDA. 


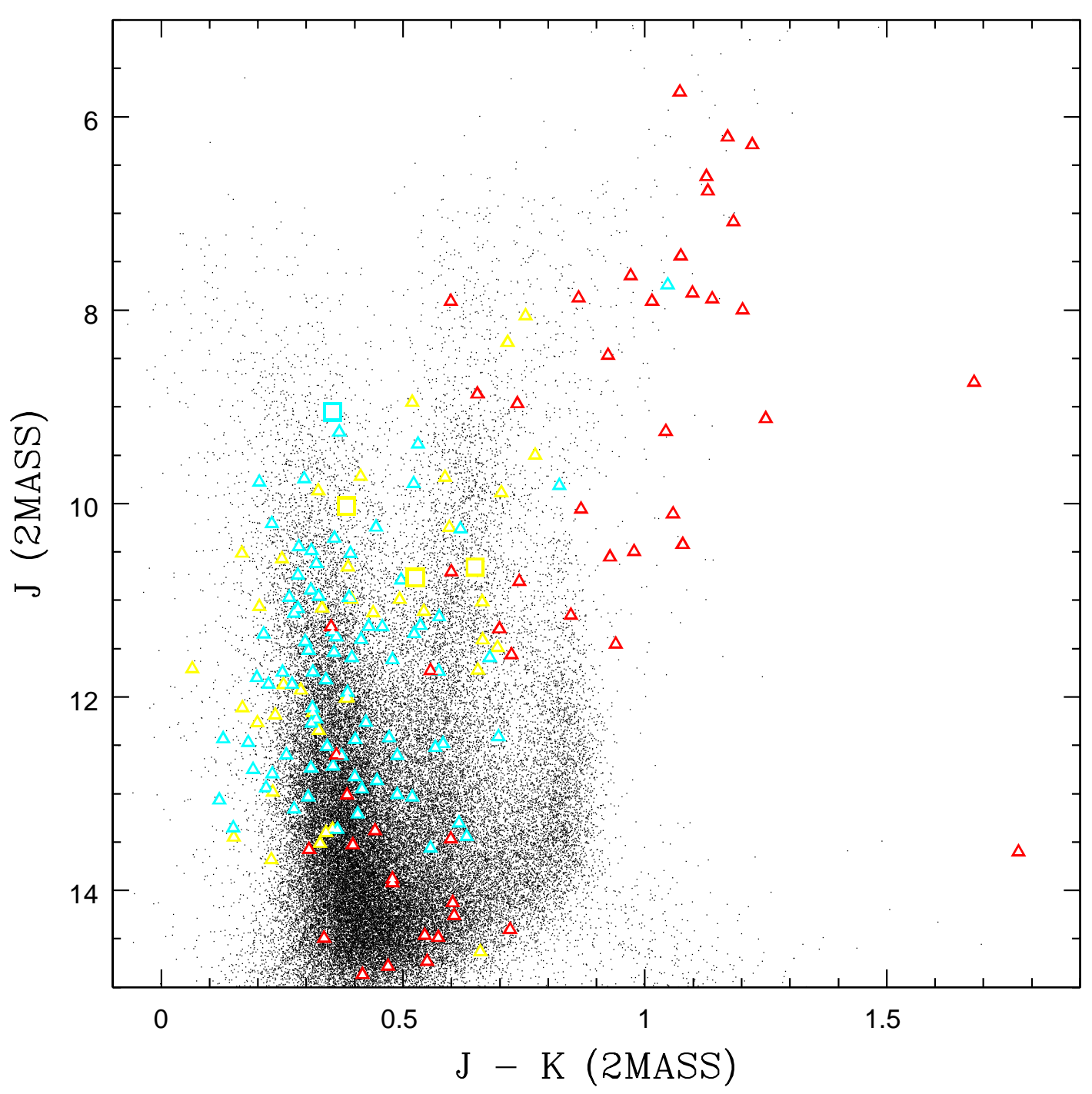

Fig. 4.7.- Color-magnitude diagram for variable and non-variable stars. Triangles plot the positions of field variables, while squares plot the four cluster variables. Red symbols mark LPVs, blue symbols mark eclipsing binaries, and yellow symbols mark pulsating variables. Non-variable stars are only plotted that match to a single 2MASS source. The eight variable stars that match to more than one 2MASS source are plotted according to the $J$ and $K$ colors of the nearest 2MASS source. The 27 variables that do not match to any 2 MASS source are not plotted. 

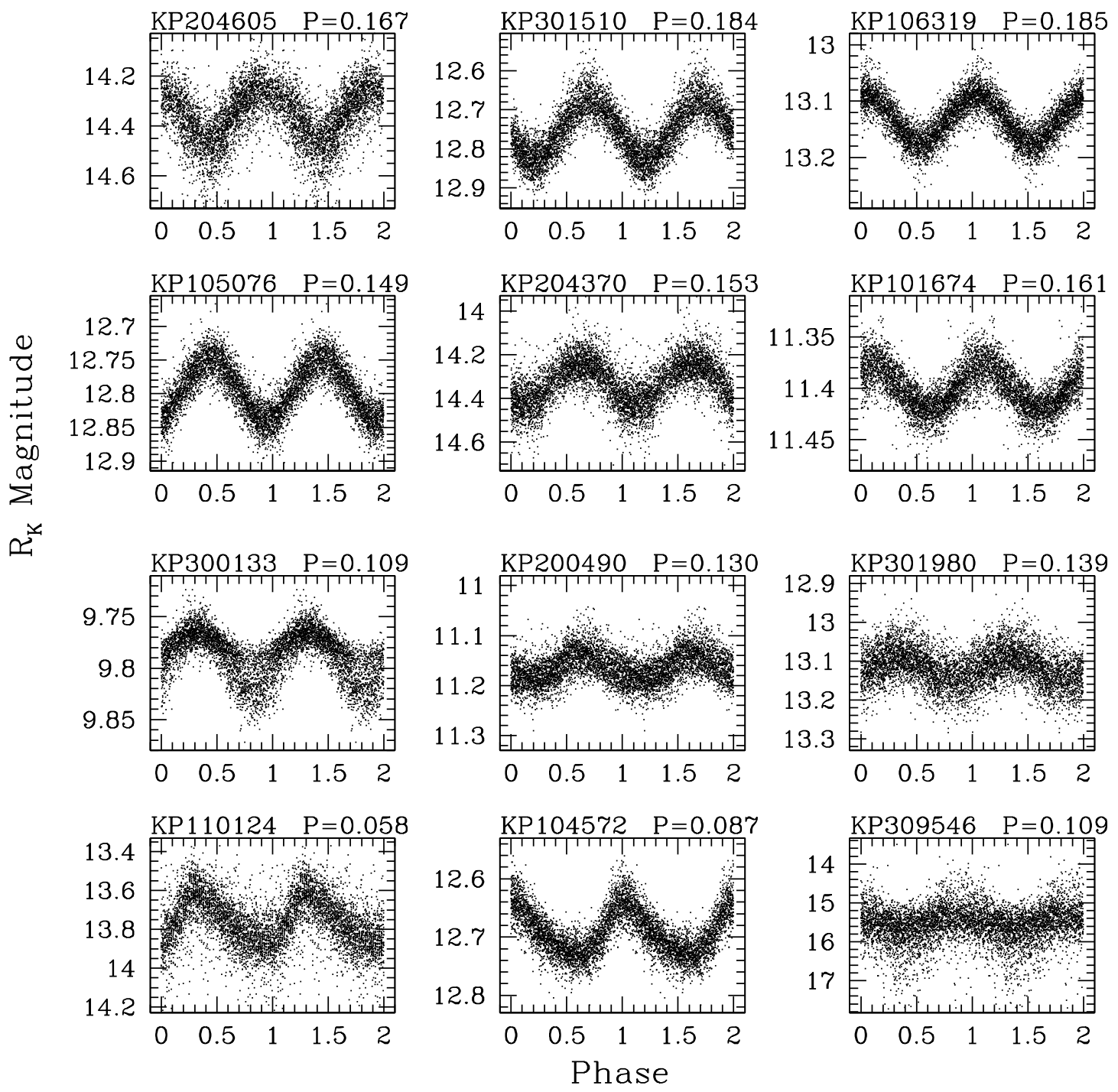

Fig. 4.8.- Lightcurves of pulsating periodic variables identified by KELT, sorted by period. 


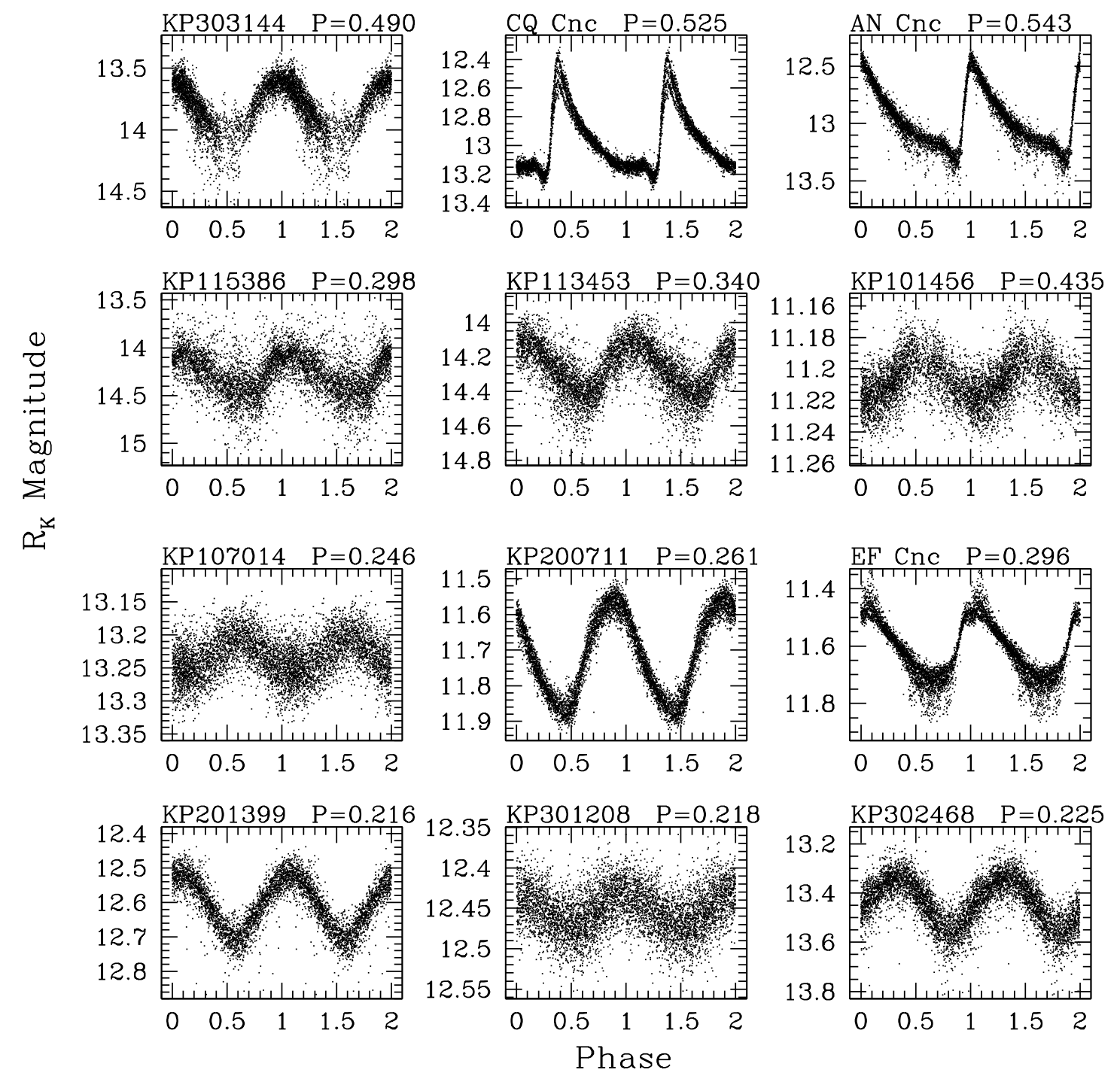

Fig. 4.9.- Lightcurves of pulsating periodic variables identified by KELT, sorted by period. 

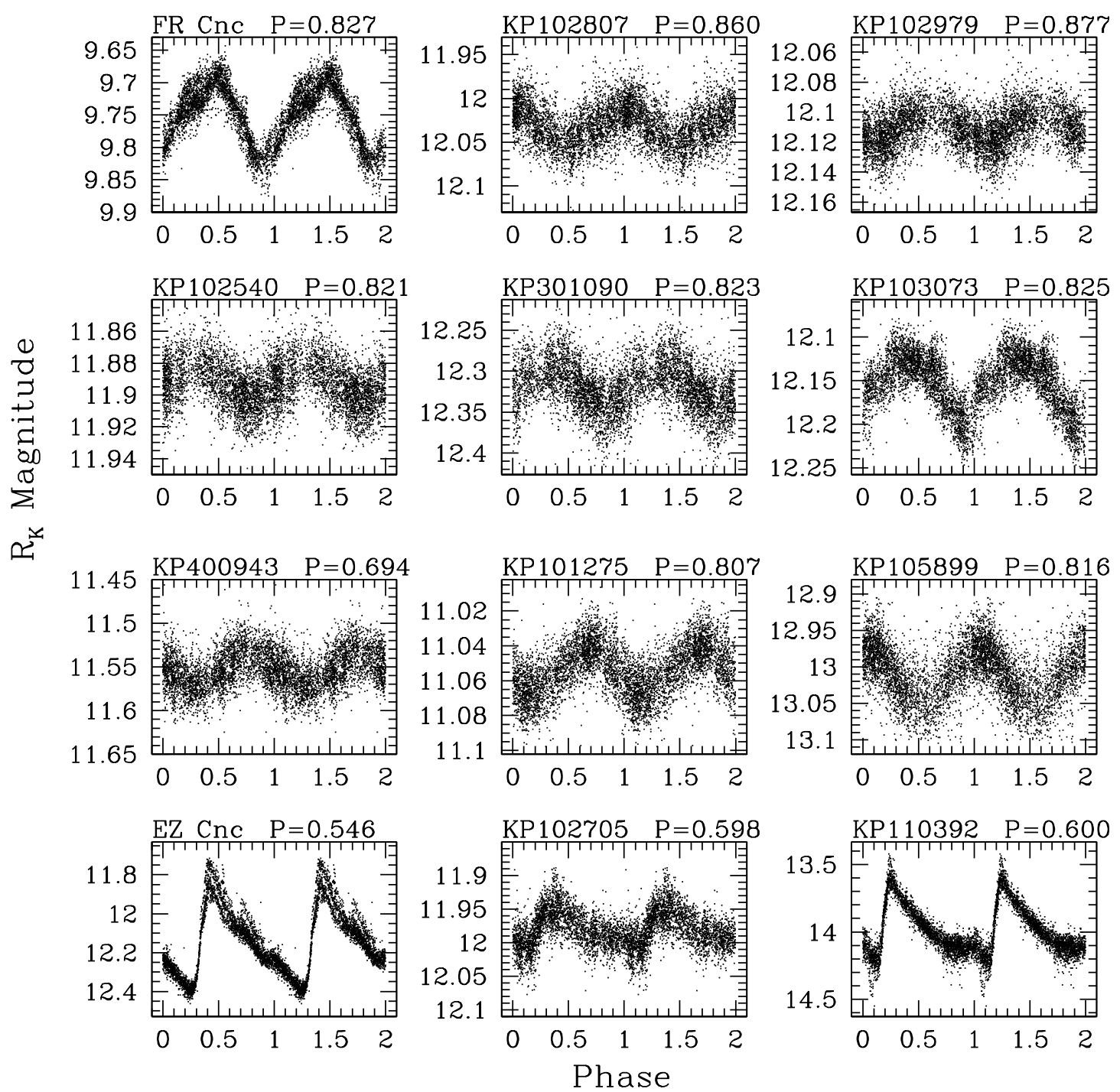

Fig. 4.10.- Lightcurves of pulsating periodic variables identified by KELT, sorted by period. 

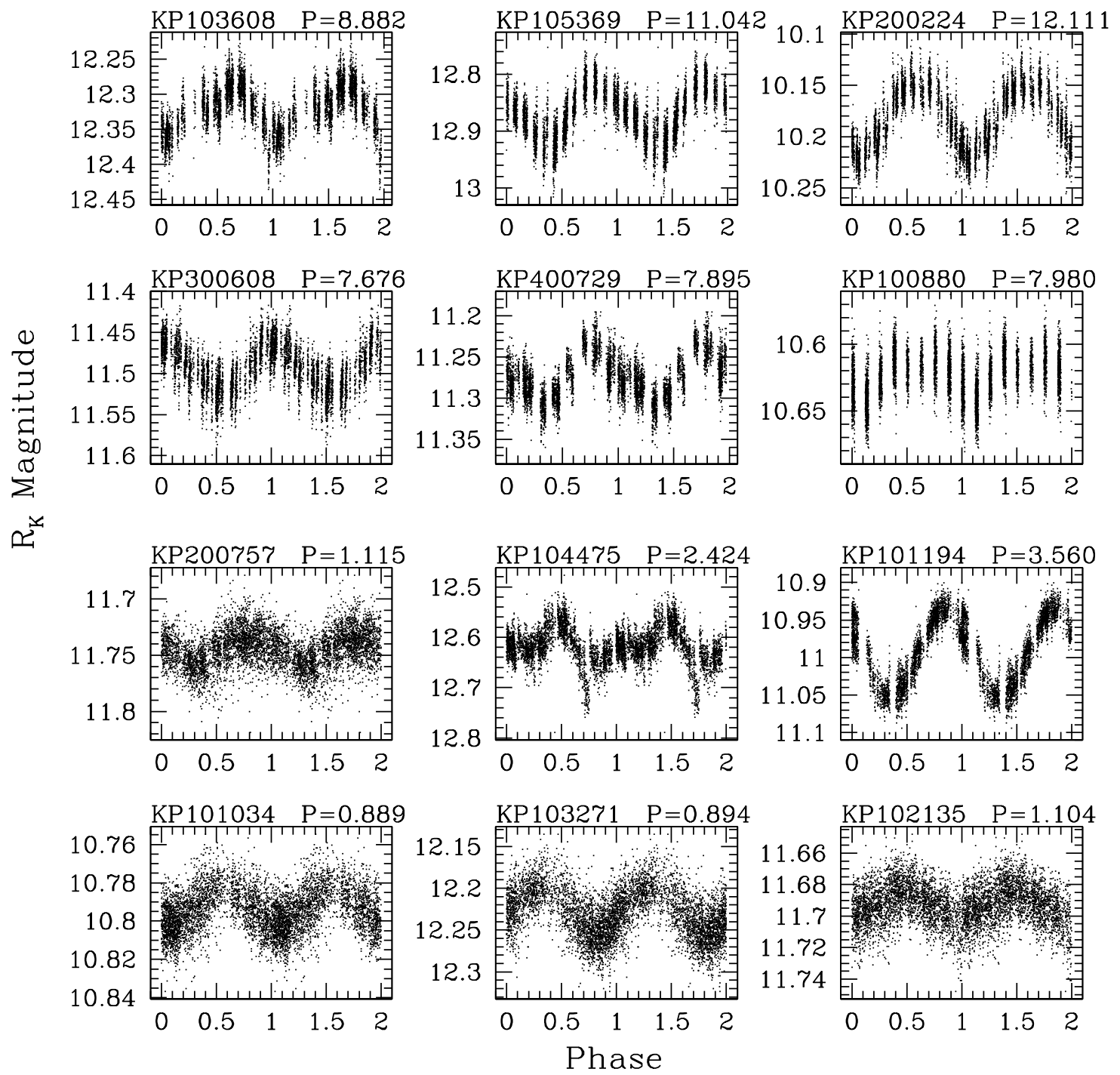

Fig. 4.11.- Lightcurves of pulsating periodic variables identified by KELT, sorted by period. 

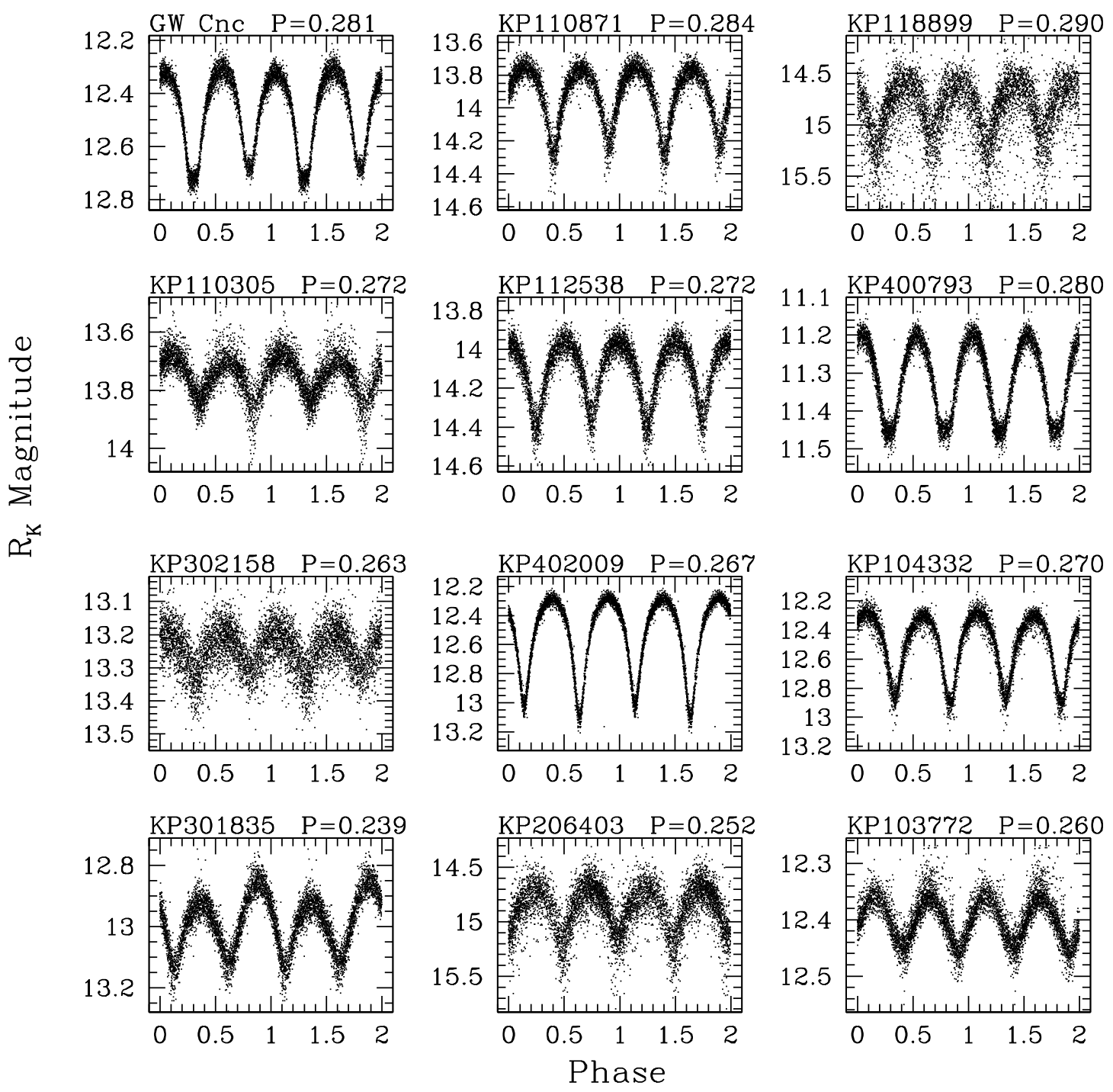

Fig. 4.12.- Lightcurves of eclipsing periodic variables identified by KELT, sorted by period. 

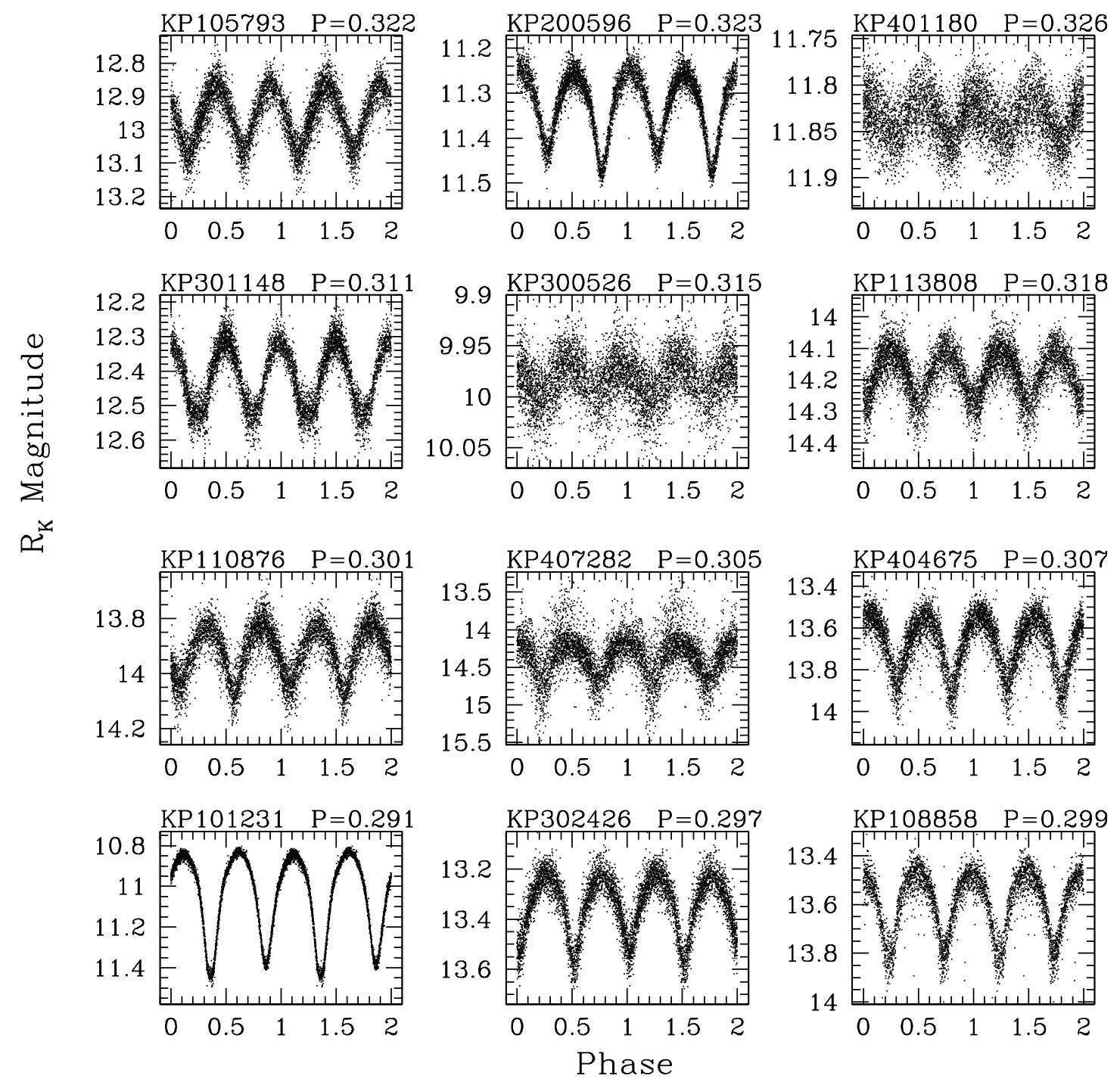

Fig. 4.13.- - Lightcurves of eclipsing periodic variables identified by KELT, sorted by period. 

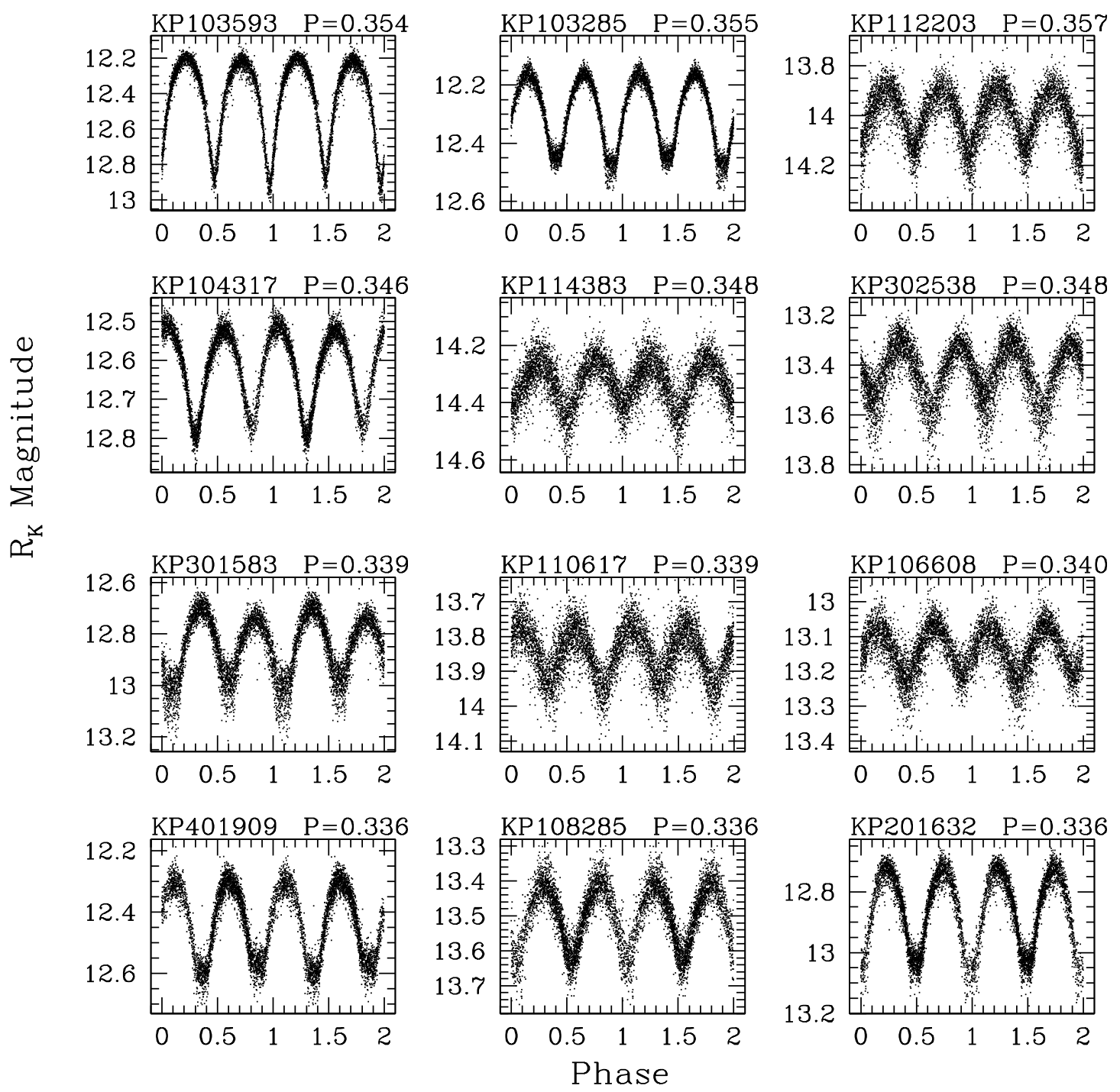

Fig. 4.14.- - Lightcurves of eclipsing periodic variables identified by KELT, sorted by period. 

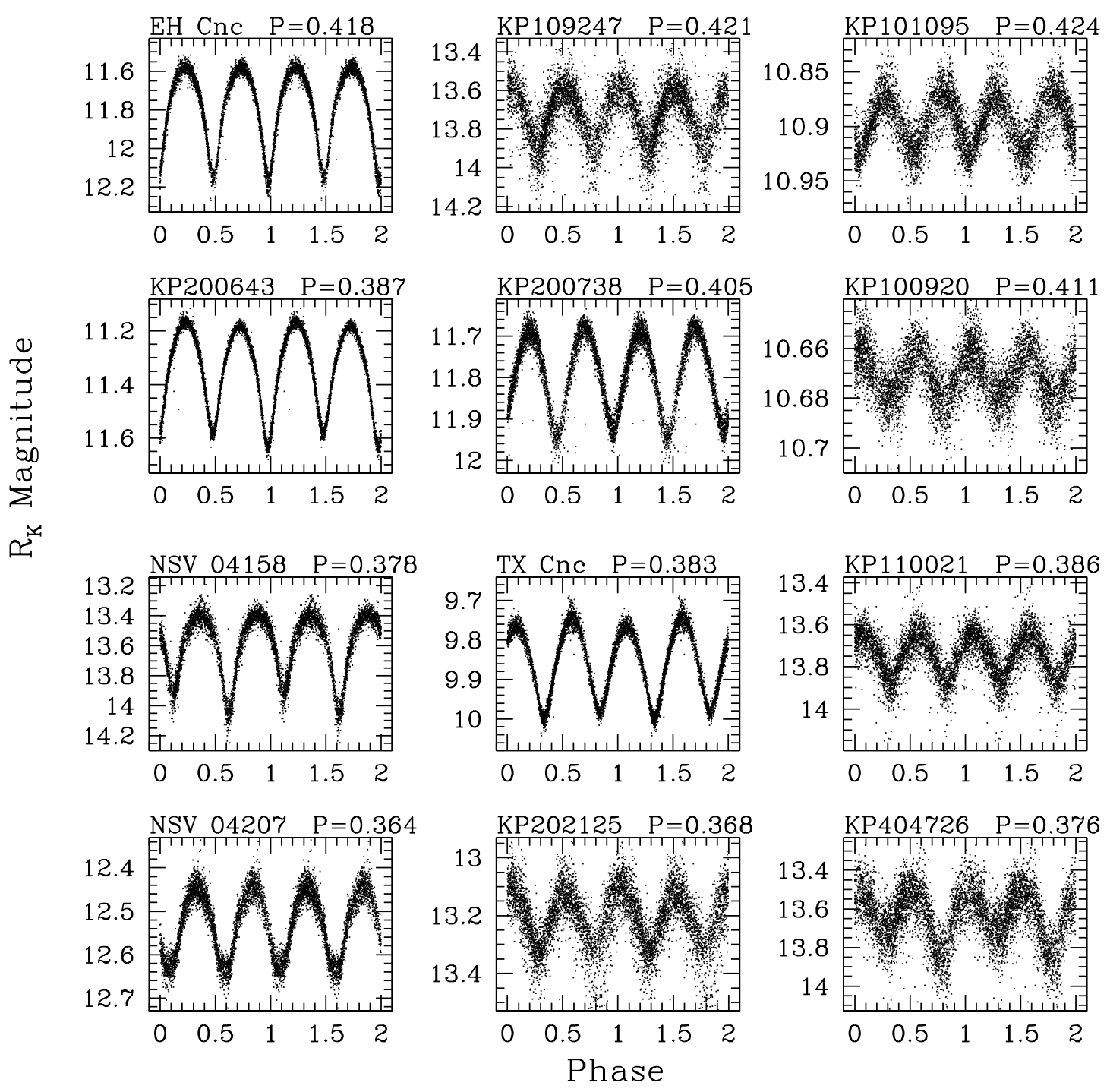

Fig. 4.15.- - Lightcurves of eclipsing periodic variables identified by KELT, sorted by period. 

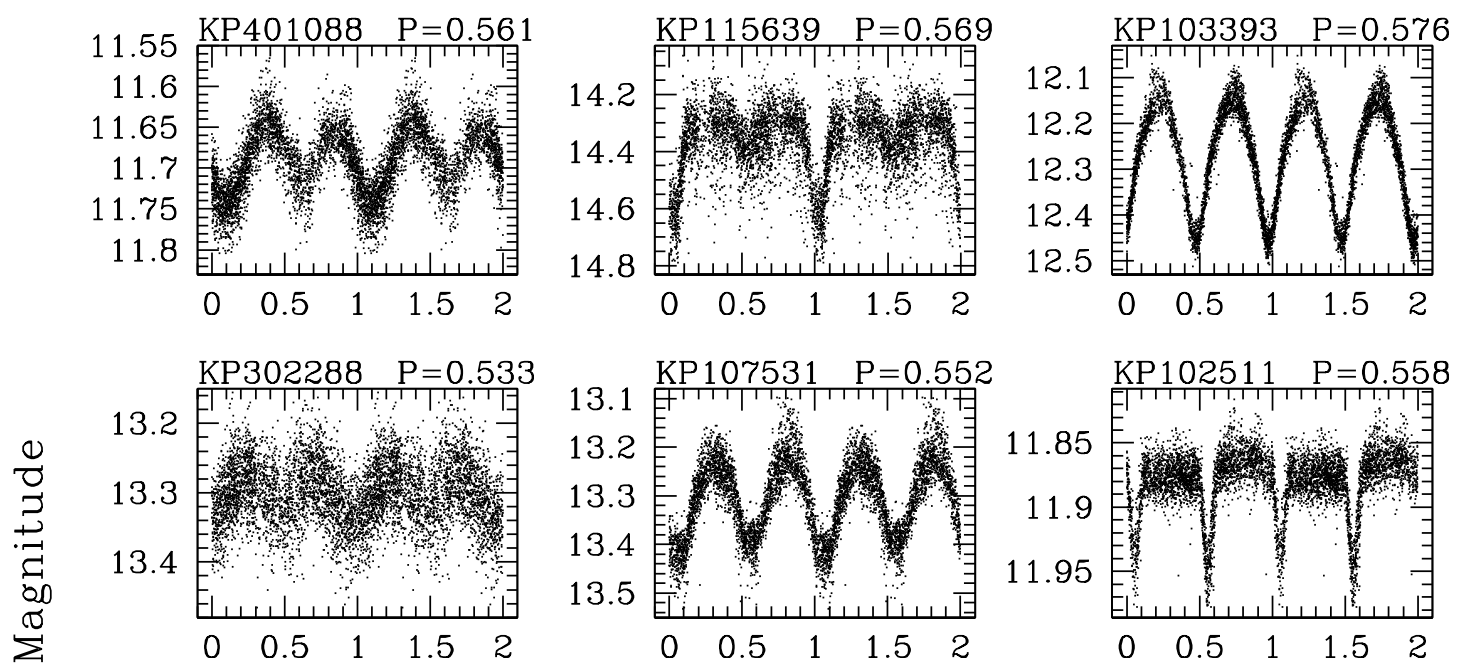

بr.
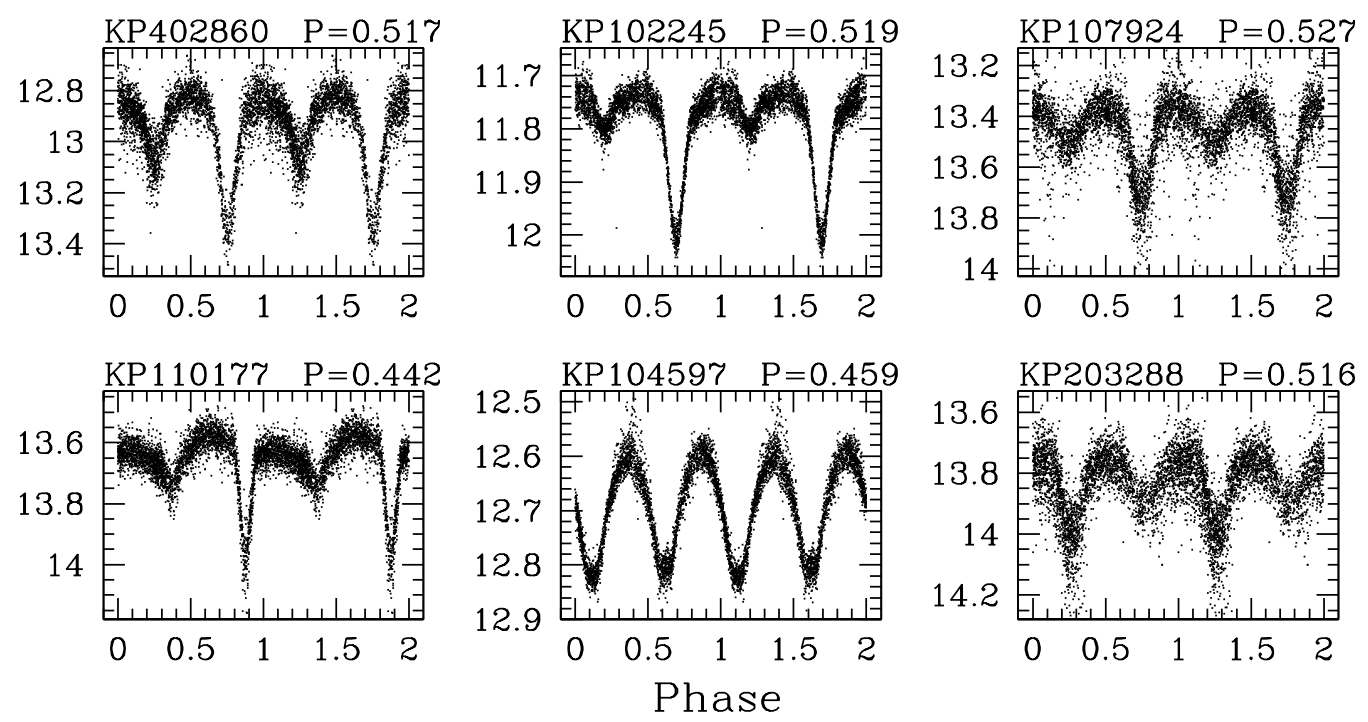

Fig. 4.16. - Lightcurves of eclipsing periodic variables identified by KELT, sorted by period. 

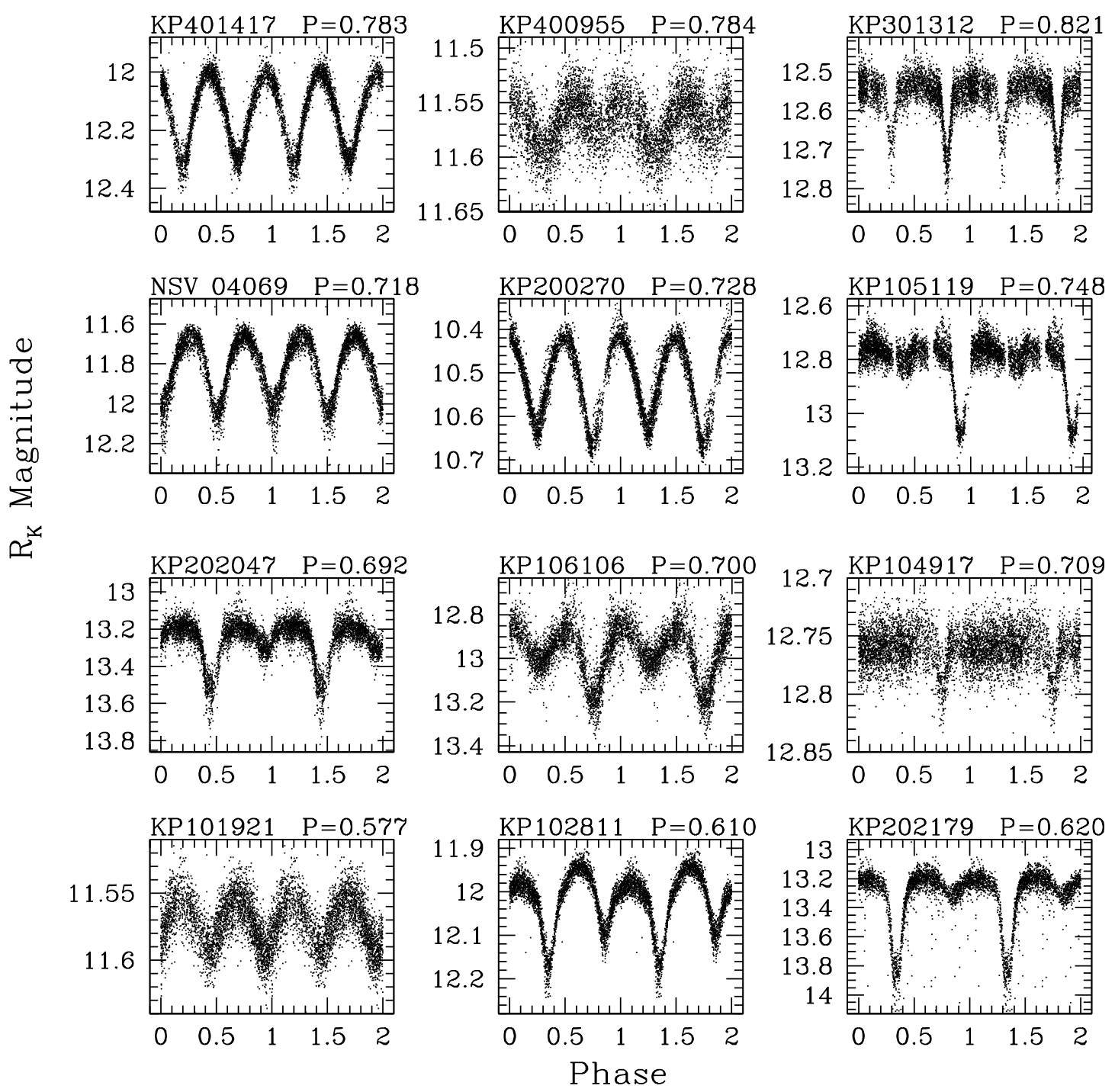

Fig. 4.17. - Lightcurves of eclipsing periodic variables identified by KELT, sorted by period. 

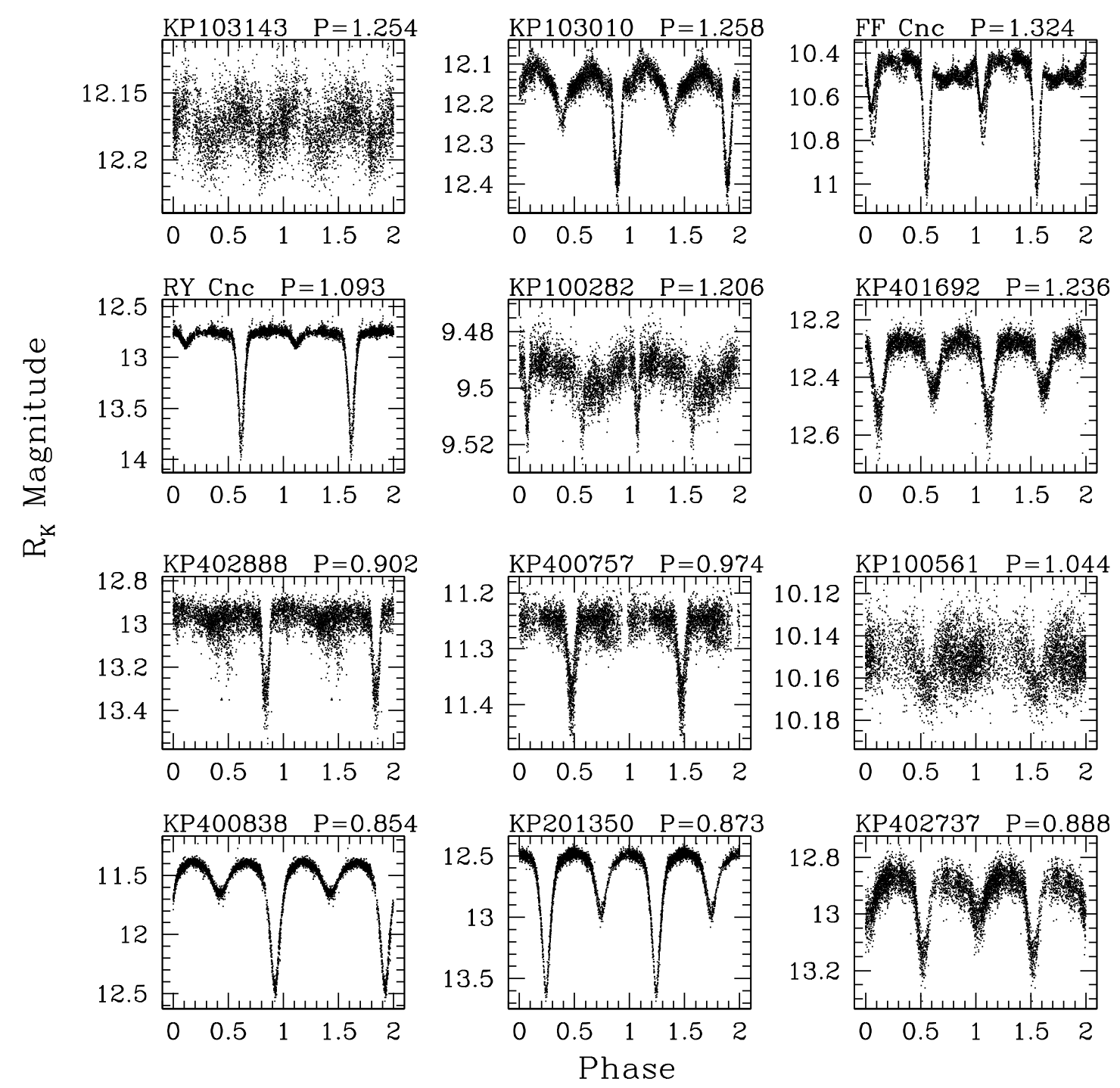

Fig. 4.18. - Lightcurves of eclipsing periodic variables identified by KELT, sorted by period. 

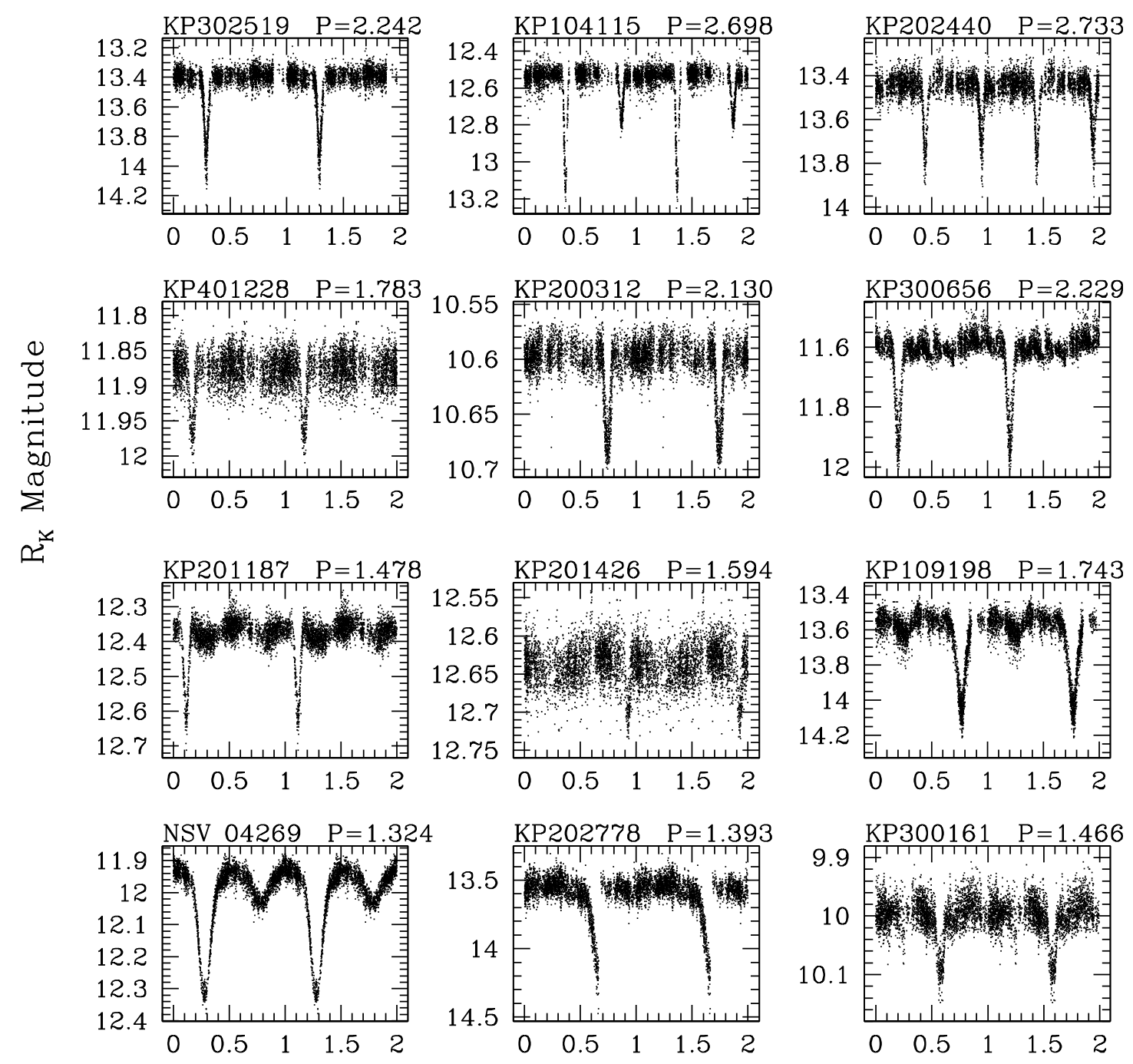

Fig. 4.19. - Lightcurves of eclipsing periodic variables identified by KELT, sorted by period.
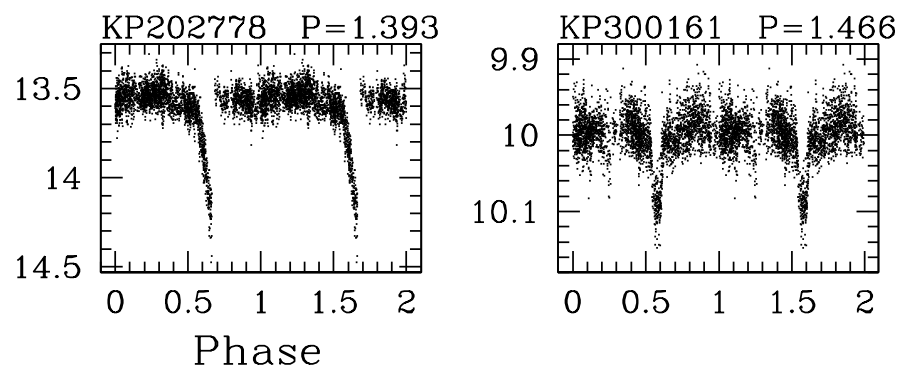

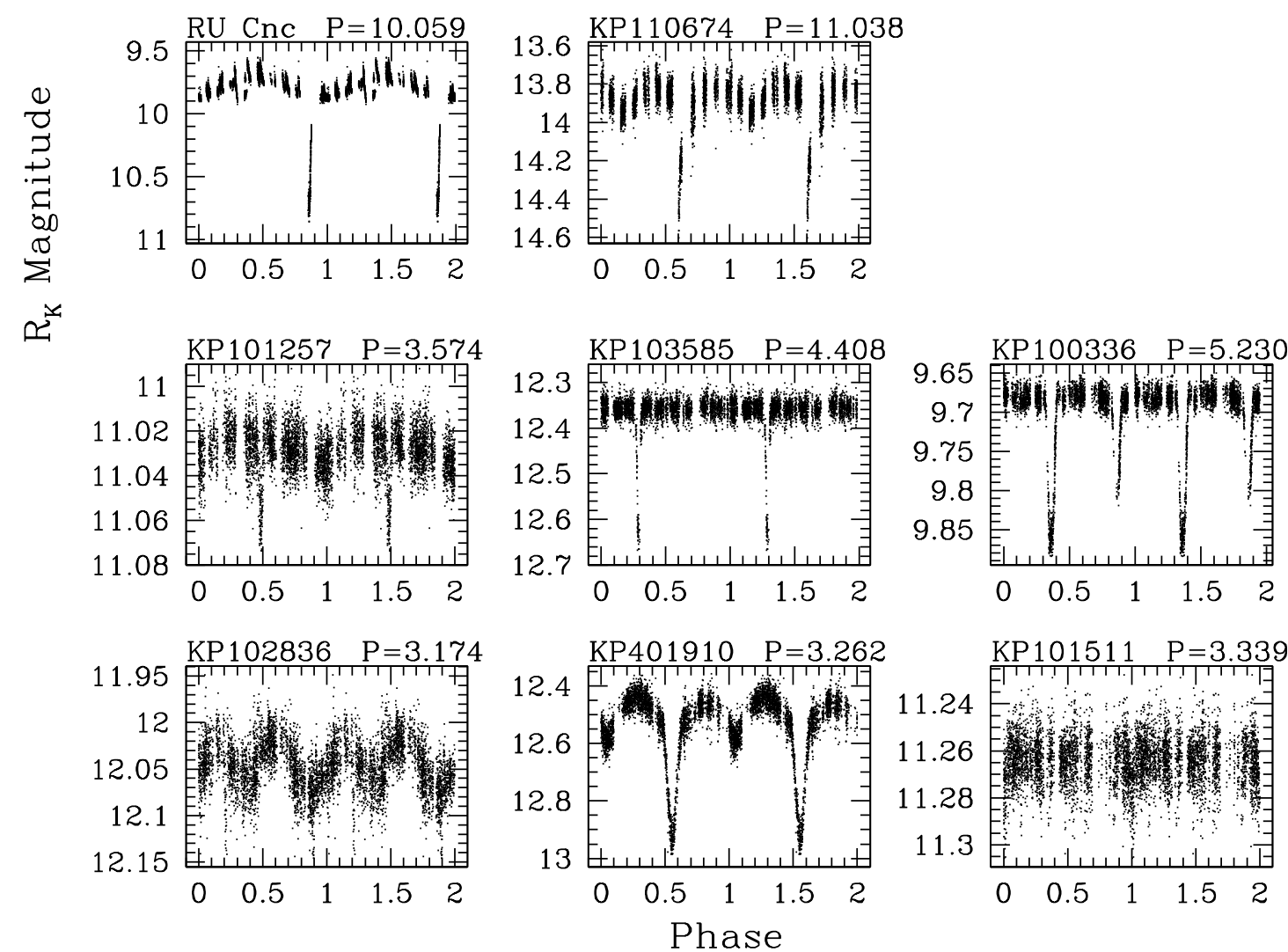

Fig. 4.20.- Lightcurves of eclipsing periodic variables identified by KELT, sorted by period. 


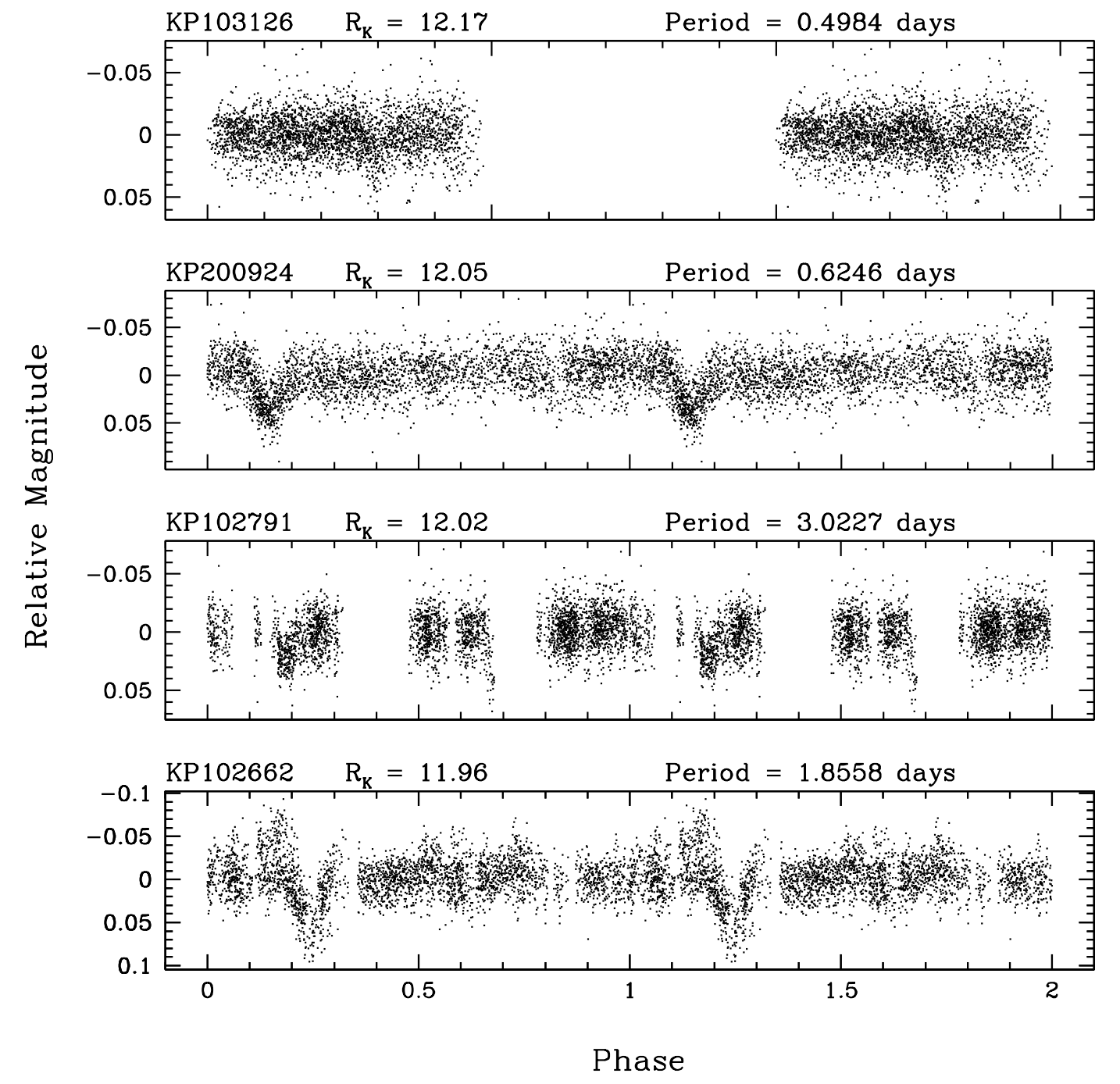

Fig. 4.21. - Four of the best transit candidates. 


\begin{tabular}{cccccccc}
\hline \hline $\begin{array}{c}\text { KELT } \\
\text { ID \# }\end{array}$ & $R_{K}$ & $\begin{array}{c}\text { RA } \\
(\mathrm{J} 2000.0)\end{array}$ & $\begin{array}{c}\text { Dec } \\
(\mathrm{J} 2000.0)\end{array}$ & $J$ & $H$ & $K$ & $\begin{array}{c}\text { 2MASS }^{\mathrm{b}} \\
\text { ID \# }\end{array}$ \\
\hline KP100169 & 9.136 & 133.01755 & 14.58464 & 6.212 & 5.349 & 5.041 & 1045.0749580 \\
KP100282 & 9.495 & 131.11816 & 20.20117 & 9.291 & 8.881 & 8.832 & $1102.0744850 *$ \\
KP100305 & 9.605 & 133.03393 & 21.84911 & 7.440 & 6.605 & 6.366 & 1118.0761240 \\
KP100306 & 9.606 & 131.14550 & 16.28497 & 7.646 & 6.826 & 6.675 & 1062.0743990 \\
KP100336 & 9.686 & 132.50840 & 17.87422 & 9.368 & 8.983 & 8.838 & $1078.0741150 *$ \\
KP100445 & 9.930 & 130.00713 & 18.99986 & 9.053 & 8.767 & 8.698 & 1089.0755560 \\
KP100561 & 10.149 & 130.99077 & 16.71554 & 7.741 & 6.932 & 6.694 & 1067.0743600 \\
KP100626 & 10.274 & 135.20934 & 18.27620 & 6.291 & 5.470 & 5.069 & 1082.0761790 \\
KP100722 & 10.408 & 131.77529 & 21.03571 & 8.869 & 8.321 & 8.215 & 1110.0755990 \\
KP100880 & 10.625 & 135.54936 & 16.38090 & 9.720 & 9.387 & 9.308 & 1063.0751000 \\
KP100886 & 10.630 & 131.78774 & 23.84970 & - & - & - & - \\
KP100920 & 10.671 & 131.08151 & 20.80536 & 9.907 & 9.638 & 9.643 & $1108.0749380 *$ \\
KP101034 & 10.796 & 130.48279 & 19.68971 & 9.869 & 9.627 & 9.544 & 1096.0752070 \\
KP101095 & 10.871 & 133.01889 & 15.36116 & 10.206 & 10.011 & 9.977 & 1053.0754740 \\
KP101194 & 10.969 & 134.29383 & 17.97599 & 9.730 & 9.240 & 9.143 & 1079.0758660 \\
KP101231 & 11.000 & 134.29044 & 18.94559 & 9.794 & 9.391 & 9.272 & 1089.0765200 \\
\hline
\end{tabular}

(cont'd)

Table 4.1. Variable Stars Identified in KELT Observations of Praesepe: Positions and 2MASS Data 
Table 4.1-Continued

\begin{tabular}{|c|c|c|c|c|c|c|c|}
\hline $\begin{array}{l}\text { KELT }^{\mathrm{a}} \\
\mathrm{ID} \#\end{array}$ & $R_{K}$ & $\begin{array}{c}\mathrm{RA} \\
(\mathrm{J} 2000.0)\end{array}$ & $\begin{array}{c}\text { Dec } \\
(\mathrm{J} 2000.0)\end{array}$ & $J$ & $H$ & $K$ & $\begin{array}{c}2 \mathrm{MASS}^{\mathrm{b}} \\
\mathrm{ID} \#\end{array}$ \\
\hline KP101257 & 11.028 & 133.13490 & 16.80922 & 11.327 & 11.044 & 10.973 & 1068.0743270 \\
\hline * KP101275 & 11.053 & 130.47658 & 19.25742 & 10.026 & 9.732 & 9.643 & 1092.0749290 \\
\hline KP101456 & 11.209 & 133.22672 & 21.98288 & 10.572 & 10.414 & 10.322 & 1119.0766810 \\
\hline KP101496 & 11.253 & 133.20090 & 18.39749 & 9.254 & 8.428 & 8.211 & 1083.0757460 \\
\hline KP101511 & 11.263 & 129.92462 & 14.08659 & 10.405 & 10.021 & 9.990 & 1040.0734660 \\
\hline KP101549 & 11.289 & 132.56274 & 23.22313 & - & - & - & - \\
\hline KP101674 & 11.402 & 132.54651 & 23.61266 & - & - & - & - \\
\hline KP101756 & 11.464 & 130.16648 & 23.26186 & - & - & - & - \\
\hline KP101831 & 11.509 & 131.82932 & 20.83213 & 10.481 & 9.999 & 9.859 & $1108.0751220 *$ \\
\hline KP101896 & 11.552 & 132.52862 & 23.52362 & - & - & - & - \\
\hline KP101921 & 11.573 & 132.90697 & 20.04515 & 10.261 & 9.730 & 9.642 & 1100.0748590 \\
\hline KP102135 & 11.692 & 133.33712 & 22.75218 & 10.656 & 10.317 & 10.270 & 1127.0749490 \\
\hline KP102245 & 11.749 & 132.76992 & 21.70140 & 10.895 & 10.622 & 10.585 & 1117.0757470 \\
\hline KP102395 & 11.819 & 134.66732 & 17.24139 & 10.707 & 10.209 & 10.107 & 1072.0749860 \\
\hline KP102511 & 11.878 & 131.20885 & 20.18795 & 9.815 & 9.174 & 8.992 & 1101.0743870 \\
\hline KP102540 & 11.894 & 130.44375 & 14.87345 & 10.991 & 10.713 & 10.598 & 1048.0737870 \\
\hline
\end{tabular}

(cont'd) 
Table 4.1-Continued

\begin{tabular}{cccccccc}
\hline \hline $\begin{array}{c}\text { KELT } \\
\text { ID \# }\end{array}$ & $R_{K}$ & $\begin{array}{c}\text { RA } \\
(J 2000.0)\end{array}$ & $\begin{array}{c}\text { Dec } \\
(J 2000.0)\end{array}$ & & $H$ & $K$ & $\begin{array}{c}\text { 2MASS } \\
\text { ID \# }\end{array}$ \\
\hline KP102588 & 11.916 & 132.71335 & 19.35729 & 11.746 & 11.554 & 11.495 & 1093.0752670 \\
KP102705 & 11.980 & 134.51968 & 22.63479 & 11.082 & 10.806 & 10.749 & 1126.0751950 \\
KP102807 & 12.030 & 132.66099 & 16.71456 & 10.993 & 10.610 & 10.500 & 1067.0747370 \\
KP102811 & 12.034 & 133.42089 & 22.34420 & 10.791 & 10.374 & 10.295 & 1123.0765180 \\
KP102836 & 12.047 & 134.25911 & 23.52545 & - & - & - & - \\
KP102908 & 12.082 & 134.96979 & 17.47890 & 10.108 & 9.268 & 9.050 & 1074.0747370 \\
KP102979 & 12.112 & 130.63708 & 21.17889 & 11.707 & 11.658 & 11.643 & 1111.0758730 \\
KP103010 & 12.124 & 130.34124 & 18.13422 & 11.430 & 11.184 & 11.132 & 1081.0745350 \\
KP103073 & 12.150 & 130.02381 & 19.02520 & 10.658 & 10.149 & 10.009 & 1090.0756590 \\
KP103143 & 12.176 & 132.41259 & 23.80157 & - & - & - & - \\
KP103254 & 12.226 & 133.24489 & 23.78463 & - & - & - & - \\
KP103267 & 12.229 & 133.18612 & 22.51454 & 10.059 & 9.452 & 9.219 & $1125.0749340 *$ \\
KP103271 & 12.232 & 131.87079 & 16.27255 & 11.116 & 10.659 & 10.573 & 1062.0745780 \\
KP103285 & 12.237 & 131.47646 & 19.58272 & 11.378 & 11.071 & 11.016 & 1095.0749450 \\
KP103393 & 12.280 & 132.90310 & 20.05444 & 11.801 & 11.626 & 11.603 & 1100.0748570 \\
KP103585 & 12.345 & 131.02744 & 18.51100 & 11.539 & 11.213 & 11.182 & 1085.0757710 \\
\hline
\end{tabular}

(cont'd) 
Table 4.1-Continued

\begin{tabular}{|c|c|c|c|c|c|c|c|}
\hline $\begin{array}{c}\text { KELT }^{\mathrm{a}} \\
\text { ID \# }\end{array}$ & $R_{K}$ & $\begin{array}{c}\text { RA } \\
(\mathrm{J} 2000.0)\end{array}$ & $\begin{array}{c}\text { Dec } \\
(\mathrm{J} 2000.0)\end{array}$ & $J$ & $H$ & $K$ & $\begin{array}{c}2 \mathrm{MASS}^{\mathrm{b}} \\
\text { ID \# }\end{array}$ \\
\hline KP103593 & 12.348 & 134.67894 & 14.88607 & 11.406 & 11.075 & 10.993 & 1048.0748330 \\
\hline KP103608 & 12.353 & 130.90608 & 19.93658 & 11.018 & 10.508 & 10.354 & 1099.0748600 \\
\hline KP103772 & 12.410 & 130.51883 & 24.10952 & - & - & - & - \\
\hline KP104115 & 12.530 & 134.02845 & 14.64294 & 11.822 & 11.520 & 11.481 & 1046.0747930 \\
\hline KP104166 & 12.549 & 130.52602 & 21.26102 & 10.554 & 9.810 & 9.626 & 1112.0755240 \\
\hline KP104174 & 12.552 & 130.67742 & 21.41570 & 11.518 & 11.259 & 11.213 & 1114.0776050 \\
\hline KP104185 & 12.557 & 132.05289 & 21.12052 & 11.272 & 10.940 & 10.843 & 1111.0761630 \\
\hline KP104317 & 12.599 & 130.33974 & 19.00734 & 11.592 & 11.293 & 11.198 & 1090.0757360 \\
\hline KP104332 & 12.602 & 135.08220 & 14.35794 & 11.736 & 11.271 & 11.162 & 1043.0750550 \\
\hline KP104424 & 12.629 & 131.62549 & 22.65176 & 10.807 & 10.190 & 10.067 & 1126.0746320 \\
\hline KP104475 & 12.644 & 132.03164 & 23.12175 & - & - & - & - \\
\hline KP104572 & 12.670 & 130.57458 & 16.39318 & 12.266 & 12.065 & 12.067 & 1063.0738790 \\
\hline KP104597 & 12.676 & 130.52484 & 21.43683 & 11.868 & 11.697 & 11.646 & 1114.0775670 \\
\hline KP104630 & 12.683 & 134.71800 & 21.07624 & 10.058 & 9.433 & 9.190 & 1110.0762010 \\
\hline KP104850 & 12.741 & 132.90945 & 16.52710 & 10.424 & 9.556 & 9.346 & 1065.0749810 \\
\hline KP104855 & 12.742 & 129.97762 & 19.82194 & 12.514 & 12.217 & 12.171 & 1098.0745650 \\
\hline
\end{tabular}

(cont'd) 
Table 4.1-Continued

\begin{tabular}{|c|c|c|c|c|c|c|c|}
\hline $\begin{array}{l}\text { KELT }^{\mathrm{a}} \\
\mathrm{ID} \#\end{array}$ & $R_{K}$ & $\begin{array}{c}\text { RA } \\
(\mathrm{J} 2000.0)\end{array}$ & $\begin{array}{c}\text { Dec } \\
(\mathrm{J} 2000.0)\end{array}$ & $J$ & $H$ & $K$ & $\begin{array}{c}2 \mathrm{MASS}^{\mathrm{b}} \\
\mathrm{ID} \#\end{array}$ \\
\hline KP104917 & 12.760 & 131.04394 & 22.25307 & 11.804 & 11.071 & 10.945 & $1122.0763630 *$ \\
\hline KP105076 & 12.804 & 130.07613 & 16.60935 & 12.014 & 11.700 & 11.632 & 1066.0737030 \\
\hline KP105119 & 12.815 & 130.94612 & 14.90738 & 12.433 & 12.299 & 12.305 & 1049.0745740 \\
\hline KP105369 & 12.877 & 130.41766 & 14.80151 & 11.489 & 10.980 & 10.794 & 1048.0737790 \\
\hline KP105756 & 12.971 & 135.26383 & 17.89894 & 11.273 & 11.051 & 10.922 & 1078.0747830 \\
\hline KP105772 & 12.975 & 131.03903 & 17.06938 & 11.729 & 11.250 & 11.172 & 1070.0742420 \\
\hline KP105793 & 12.979 & 132.74935 & 13.96247 & 12.230 & 11.954 & 11.909 & 1039.0746360 \\
\hline KP105899 & 13.005 & 133.45578 & 20.85212 & 11.412 & 10.845 & 10.747 & 1108.0754490 \\
\hline KP106106 & 13.054 & 135.21007 & 23.62369 & - & - & - & - \\
\hline KP106218 & 13.077 & 133.14519 & 22.48431 & 11.156 & 10.453 & 10.309 & 1124.0755070 \\
\hline KP106227 & 13.080 & 133.37819 & 14.13207 & 11.563 & 11.003 & 10.839 & 1041.0741190 \\
\hline KP106319 & 13.096 & 130.44779 & 21.97142 & 12.161 & 11.909 & 11.847 & 1119.0760800 \\
\hline KP106351 & 13.102 & 134.54297 & 15.80525 & 12.112 & 11.941 & 11.944 & 1058.0757020 \\
\hline KP106452 & 13.122 & 131.34256 & 15.27477 & 12.187 & 11.994 & 11.951 & 1052.0750150 \\
\hline KP106608 & 13.157 & 133.67184 & 19.11521 & 12.352 & 12.038 & 11.998 & $1091.0753150 *$ \\
\hline KP106885 & 13.213 & 133.82978 & 16.43977 & 11.455 & 10.694 & 10.515 & 1064.0744910 \\
\hline
\end{tabular}

(cont'd) 
Table 4.1-Continued

\begin{tabular}{|c|c|c|c|c|c|c|c|}
\hline $\begin{array}{c}\text { KELT }^{\mathrm{a}} \\
\text { ID \# }\end{array}$ & $R_{K}$ & $\begin{array}{c}\text { RA } \\
(\mathrm{J} 2000.0)\end{array}$ & $\begin{array}{c}\text { Dec } \\
(\mathrm{J} 2000.0)\end{array}$ & $J$ & $H$ & $K$ & $\begin{array}{c}2 \mathrm{MASS}^{\mathrm{b}} \\
\text { ID \# }\end{array}$ \\
\hline KP107014 & 13.234 & 130.22716 & 14.42231 & 12.345 & 12.011 & 12.019 & 1044.0741240 \\
\hline KP107531 & 13.333 & 129.98160 & 23.33538 & - & - & - & - \\
\hline KP107924 & 13.408 & 135.19565 & 15.42220 & 12.941 & 12.796 & 12.724 & 1054.0756940 \\
\hline KP108285 & 13.473 & 130.92216 & 18.42757 & 12.735 & 12.477 & 12.425 & 1084.0761990 \\
\hline KP108858 & 13.569 & 130.25118 & 13.67656 & 12.607 & 12.213 & 12.119 & 1036.0747170 \\
\hline KP109198 & 13.619 & 133.45391 & 21.49120 & 13.066 & 12.953 & 12.946 & 1114.0781610 \\
\hline KP109247 & 13.625 & 133.88598 & 14.54473 & 13.160 & 12.925 & 12.885 & 1045.0751940 \\
\hline KP110021 & 13.734 & 132.80862 & 16.07745 & 13.036 & 12.801 & 12.732 & 1060.0746020 \\
\hline KP110124 & 13.751 & 134.72799 & 15.36940 & 13.450 & 13.295 & 13.300 & 1053.0759220 \\
\hline KP110177 & 13.760 & 130.17535 & 14.98393 & 12.410 & 11.867 & 11.713 & 1049.0743760 \\
\hline KP110305 & 13.777 & 130.52273 & 22.41861 & 12.483 & 12.007 & 11.900 & 1124.0749670 \\
\hline KP110392 & 13.792 & 130.19981 & 15.41455 & 13.402 & 13.115 & 13.061 & 1054.0744870 \\
\hline KP110617 & 13.826 & 131.51008 & 15.44463 & - & - & - & - \\
\hline KP110674 & 13.837 & 133.33587 & 23.52222 & - & - & - & - \\
\hline KP110871 & 13.864 & 130.70418 & 15.39379 & 12.524 & 12.010 & 11.957 & 1053.0748840 \\
\hline KP110876 & 13.865 & 131.03416 & 16.84885 & 12.866 & 12.492 & 12.420 & 1068.0738120 \\
\hline
\end{tabular}

(cont'd) 
Table 4.1-Continued

\begin{tabular}{cccccccc}
\hline \hline $\begin{array}{c}\text { KELT } \\
\text { ID \# }\end{array}$ & $R_{K}$ & $\begin{array}{c}\text { RA } \\
(\mathrm{J} 2000.0)\end{array}$ & $\begin{array}{c}\text { Dec } \\
(\mathrm{J} 2000.0)\end{array}$ & & & & \\
KP111363 & 13.933 & 134.00672 & 18.27980 & - & - & - & 2MASS \\
KP112203 & 14.042 & 132.87884 & 21.75790 & 13.009 & 12.588 & 12.521 & 1117.0757790 \\
KP112538 & 14.086 & 129.95615 & 15.42027 & 13.304 & 12.770 & 12.688 & 1054.0744100 \\
KP113453 & 14.197 & 132.70565 & 16.31045 & 13.683 & 13.550 & 13.455 & 1063.0743930 \\
KP113808 & 14.236 & 131.51525 & 20.96654 & 13.211 & 12.920 & 12.805 & 1109.0750100 \\
KP114383 & 14.301 & 131.15271 & 21.75311 & 13.371 & 13.095 & 13.007 & 1117.0754300 \\
KP114757 & 14.344 & 133.70368 & 20.10852 & 13.602 & 12.676 & 11.830 & 1101.0749620 \\
KP115386 & 14.408 & 134.78593 & 23.89257 & - & - & - & - \\
KP115639 & 14.434 & 131.93564 & 23.76530 & - & - & - & - \\
KP115973 & 14.469 & 135.41519 & 16.40212 & 13.531 & 13.232 & 13.135 & 1064.0748100 \\
KP118312 & 14.698 & 132.30275 & 20.90523 & 13.923 & 13.617 & 13.445 & 1109.0751930 \\
KP118899 & 14.754 & 134.88939 & 21.16955 & 13.444 & 12.955 & 12.812 & 1111.0767430 \\
KP119499 & 14.808 & 135.31678 & 19.63988 & 13.468 & 12.922 & 12.869 & 1096.0763660 \\
KP121436 & 14.975 & 130.64868 & 20.64923 & 13.881 & 13.436 & 13.403 & 1106.0753000 \\
KP126266 & 15.351 & 132.71161 & 19.68410 & 14.129 & 13.679 & 13.526 & 1096.0757440 \\
KP127604 & 15.455 & 131.49181 & 19.93790 & 14.733 & 14.361 & 14.183 & 1099.0749840 \\
\hline
\end{tabular}

(cont'd) 
Table 4.1-Continued

\begin{tabular}{|c|c|c|c|c|c|c|c|}
\hline $\begin{array}{c}\text { KELT }^{\mathrm{a}} \\
\text { ID \# }\end{array}$ & $R_{K}$ & $\begin{array}{c}\text { RA } \\
(\mathrm{J} 2000.0)\end{array}$ & $\begin{array}{c}\text { Dec } \\
(\mathrm{J} 2000.0)\end{array}$ & $J$ & $H$ & $K$ & $\begin{array}{c}2 \mathrm{MASS}^{\mathrm{b}} \\
\text { ID \# }\end{array}$ \\
\hline KP127745 & 15.466 & 132.14931 & 20.92373 & 14.871 & 14.607 & 14.455 & 1109.0751580 \\
\hline KP128381 & 15.511 & 132.94836 & 16.20497 & 14.488 & 14.005 & 13.915 & 1062.0748320 \\
\hline KP130733 & 15.689 & 131.45529 & 20.12358 & 14.260 & 13.725 & 13.654 & 1101.0744500 \\
\hline KP131942 & 15.791 & 135.36885 & 18.02716 & 14.784 & 14.285 & 14.315 & 1080.0759520 \\
\hline KP133934 & 16.024 & 131.81019 & 19.93585 & 14.405 & 13.792 & 13.684 & 1099.0750680 \\
\hline KP200074 & 9.079 & 129.40268 & 16.02737 & 5.746 & 4.835 & 4.674 & 1060.0737090 \\
\hline KP200152 & 9.711 & 128.95538 & 21.21765 & 7.873 & 7.189 & 7.010 & 1112.0751470 \\
\hline KP200188 & 10.019 & 129.38447 & 23.55798 & - & - & - & - \\
\hline KP200191 & 10.024 & 128.87199 & 15.14900 & 7.086 & 6.216 & 5.903 & 1051.0742960 \\
\hline KP200210 & 10.116 & 129.65732 & 22.76973 & 7.908 & 7.128 & 6.893 & 1127.0741350 \\
\hline KP200224 & 10.184 & 128.88365 & 17.05935 & 8.954 & 8.538 & 8.435 & 1070.0736830 \\
\hline KP200244 & 10.312 & 128.68203 & 23.04457 & - & - & - & - \\
\hline KP200257 & 10.384 & 129.60230 & 20.50270 & 8.466 & 7.701 & 7.542 & 1105.0747150 \\
\hline KP200262 & 10.408 & 129.66094 & 21.42135 & 8.969 & 8.400 & 8.233 & 1114.0773630 \\
\hline KP200270 & 10.453 & 128.69299 & 17.76642 & 9.779 & 9.611 & 9.576 & 1077.0731030 \\
\hline KP200312 & 10.600 & 129.46961 & 16.36575 & 9.745 & 9.498 & 9.449 & 1063.0736000 \\
\hline
\end{tabular}

(cont'd) 
Table 4.1-Continued

\begin{tabular}{|c|c|c|c|c|c|c|c|}
\hline $\begin{array}{l}\text { KELT }^{\mathrm{a}} \\
\mathrm{ID} \#\end{array}$ & $R_{K}$ & $\begin{array}{c}\text { RA } \\
(\mathrm{J} 2000.0)\end{array}$ & $\begin{array}{c}\text { Dec } \\
(\mathrm{J} 2000.0)\end{array}$ & $J$ & $H$ & $K$ & $\begin{array}{c}2 \mathrm{MASS}^{\mathrm{b}} \\
\mathrm{ID} \#\end{array}$ \\
\hline KP200490 & 11.146 & 128.55033 & 16.73073 & 10.514 & 10.351 & 10.347 & 1067.0737230 \\
\hline KP200596 & 11.385 & 128.73938 & 19.91683 & 10.245 & 9.884 & 9.801 & 1099.0742660 \\
\hline KP200643 & 11.489 & 128.56992 & 13.98220 & 10.487 & 10.231 & 10.176 & 1039.0734620 \\
\hline KP200711 & 11.642 & 129.94680 & 14.29008 & 11.065 & 10.910 & 10.862 & 1042.0738940 \\
\hline KP200738 & 11.701 & 129.30049 & 13.84782 & 11.087 & 10.834 & 10.804 & 1038.0739890 \\
\hline * KP200757 & 11.745 & 129.90634 & 18.17039 & 10.763 & 10.321 & 10.237 & 1081.0744290 \\
\hline KP201187 & 12.361 & 128.96040 & 20.55906 & 11.273 & 10.925 & 10.816 & 1105.0745450 \\
\hline KP201350 & 12.543 & 129.40514 & 14.59862 & 11.346 & 10.913 & 10.823 & 1045.0739360 \\
\hline KP201399 & 12.596 & 129.91210 & 13.72237 & 11.930 & 11.726 & 11.641 & 1037.0739920 \\
\hline KP201426 & 12.636 & 128.77766 & 15.86104 & 11.954 & 11.627 & 11.569 & 1058.0741870 \\
\hline KP201594 & 12.800 & 129.32782 & 17.04804 & 11.297 & 10.709 & 10.598 & 1070.0737980 \\
\hline KP201632 & 12.837 & 129.79576 & 16.79492 & 12.113 & 11.892 & 11.800 & 1067.0740580 \\
\hline KP202047 & 13.178 & 128.80993 & 23.68985 & - & - & - & - \\
\hline KP202125 & 13.224 & 129.87202 & 23.58952 & - & - & - & - \\
\hline KP202179 & 13.269 & 129.64794 & 13.70668 & 12.749 & 12.599 & 12.559 & 1037.0738980 \\
\hline KP202440 & 13.435 & 128.73262 & 22.55996 & 12.599 & 12.355 & 12.340 & 1125.0739420 \\
\hline
\end{tabular}

(cont'd) 
Table 4.1-Continued

\begin{tabular}{|c|c|c|c|c|c|c|c|}
\hline $\begin{array}{l}\text { KELT }^{\mathrm{a}} \\
\mathrm{ID} \#\end{array}$ & $R_{K}$ & $\begin{array}{c}\text { RA } \\
(\mathrm{J} 2000.0)\end{array}$ & $\begin{array}{c}\text { Dec } \\
(\mathrm{J} 2000.0)\end{array}$ & $J$ & $H$ & $K$ & $\begin{array}{c}2 \mathrm{MASS}^{\mathrm{b}} \\
\mathrm{ID} \#\end{array}$ \\
\hline KP202483 & 13.459 & 129.83068 & 15.51133 & 12.599 & 12.285 & 12.237 & 1055.0741610 \\
\hline KP202655 & 13.556 & 129.50897 & 16.99027 & 12.423 & 12.060 & 11.952 & 1069.0738920 \\
\hline KP202778 & 13.608 & 128.97139 & 20.91898 & 12.825 & 12.476 & 12.425 & 1109.0744050 \\
\hline KP202994 & 13.717 & 129.72676 & 21.17214 & 13.013 & 12.676 & 12.629 & 1111.0756810 \\
\hline KP203288 & 13.858 & 129.75875 & 15.25845 & 13.352 & 13.208 & 13.203 & 1052.0746010 \\
\hline KP204370 & 14.282 & 128.39201 & 22.06084 & 13.515 & 13.231 & 13.187 & 1120.0752690 \\
\hline KP204605 & 14.381 & 128.40400 & 18.16221 & 13.367 & 13.008 & 13.013 & 1081.0740610 \\
\hline KP206403 & 14.927 & 129.55466 & 17.42242 & 13.564 & 13.086 & 13.007 & 1074.0734640 \\
\hline KP300063 & 9.219 & 128.25422 & 17.22665 & 7.909 & 7.416 & 7.310 & 1072.0733840 \\
\hline KP300133 & 9.785 & 127.86619 & 19.88428 & 8.335 & 7.739 & 7.619 & 1098.0739790 \\
\hline KP300135 & 9.788 & 128.12730 & 15.82408 & 8.060 & 7.456 & 7.307 & 1058.0740180 \\
\hline KP300161 & 9.982 & 128.00962 & 19.60058 & 9.263 & 8.970 & 8.895 & 1096.0744840 \\
\hline KP300277 & 10.581 & 127.41380 & 17.28352 & 9.384 & 8.939 & 8.853 & 1072.0731650 \\
\hline KP300434 & 11.088 & 127.15242 & 21.93378 & 7.999 & 7.135 & 6.797 & 1119.0752650 \\
\hline KP300526 & 11.290 & 127.00145 & 22.93127 & - & - & - & - \\
\hline KP300603 & 11.482 & 126.91865 & 19.26223 & 7.825 & 7.091 & 6.727 & 1092.0739200 \\
\hline
\end{tabular}

(cont'd) 
Table 4.1-Continued

\begin{tabular}{|c|c|c|c|c|c|c|c|}
\hline $\begin{array}{l}\text { KELT }^{\mathrm{a}} \\
\mathrm{ID} \#\end{array}$ & $R_{K}$ & $\begin{array}{c}\text { RA } \\
(\mathrm{J} 2000.0)\end{array}$ & $\begin{array}{c}\text { Dec } \\
(\mathrm{J} 2000.0)\end{array}$ & $J$ & $H$ & $K$ & $\begin{array}{c}2 \mathrm{MASS}^{\mathrm{b}} \\
\mathrm{ID} \#\end{array}$ \\
\hline KP300608 & 11.496 & 127.35241 & 14.36576 & 9.887 & 9.313 & 9.184 & 1043.0730130 \\
\hline KP300656 & 11.603 & 127.85822 & 20.91667 & 10.970 & 10.680 & 10.582 & 1109.0741130 \\
\hline KP300678 & 11.658 & 127.31299 & 18.38538 & 8.748 & 7.709 & 7.067 & 1083.0742580 \\
\hline KP300976 & 12.169 & 127.09836 & 19.97421 & 9.123 & 8.204 & 7.873 & 1099.0738550 \\
\hline KP301090 & 12.320 & 127.51882 & 16.73953 & 11.129 & 10.777 & 10.691 & 1067.0734550 \\
\hline KP301148 & 12.393 & 128.17451 & 23.55348 & - & - & - & - \\
\hline KP301208 & 12.453 & 127.73636 & 21.16272 & 11.874 & 11.676 & 11.621 & 1111.0751700 \\
\hline KP301312 & 12.545 & 127.28293 & 20.57023 & 11.742 & 11.484 & 11.428 & 1105.0741250 \\
\hline KP301510 & 12.736 & 127.20627 & 18.42707 & 12.006 & 11.695 & 11.621 & 1084.0752170 \\
\hline KP301583 & 12.805 & 127.09350 & 19.16872 & 11.616 & 11.278 & 11.138 & 1091.0737930 \\
\hline KP301835 & 13.020 & 127.01759 & 17.99201 & 11.593 & 11.050 & 10.914 & 1079.0740120 \\
\hline KP301980 & 13.128 & 127.99386 & 17.38058 & 11.722 & 11.175 & 11.067 & 1073.0731580 \\
\hline KP302158 & 13.233 & 127.90973 & 15.20447 & 12.264 & 11.932 & 11.841 & 1052.0740720 \\
\hline KP302288 & 13.300 & 127.25518 & 20.34976 & 12.714 & 12.432 & 12.359 & 1103.0731870 \\
\hline KP302426 & 13.382 & 128.43628 & 15.32971 & 13.087 & 12.565 & 12.511 & $1053.0742410 *$ \\
\hline KP302468 & 13.405 & 128.16966 & 17.84913 & 12.984 & 12.788 & 12.753 & 1078.0730730 \\
\hline
\end{tabular}

(cont'd) 
Table 4.1-Continued

\begin{tabular}{|c|c|c|c|c|c|c|c|}
\hline $\begin{array}{l}\text { KELT }^{\mathrm{a}} \\
\mathrm{ID} \#\end{array}$ & $R_{K}$ & $\begin{array}{c}\text { RA } \\
(\mathrm{J} 2000.0)\end{array}$ & $\begin{array}{c}\text { Dec } \\
(\mathrm{J} 2000.0)\end{array}$ & $J$ & $H$ & $K$ & $\begin{array}{c}2 \mathrm{MASS}^{\mathrm{b}} \\
\mathrm{ID} \#\end{array}$ \\
\hline KP302519 & 13.437 & 127.99812 & 21.41259 & 12.441 & 12.117 & 12.040 & 1114.0769350 \\
\hline KP302538 & 13.448 & 128.07795 & 17.83218 & 12.612 & 12.286 & 12.239 & 1078.0730540 \\
\hline KP303144 & 13.747 & 128.28988 & 23.46403 & - & - & - & - \\
\hline KP303492 & 13.890 & 127.32312 & 21.34491 & 13.578 & 13.279 & 13.272 & 1113.0765330 \\
\hline KP309546 & 15.612 & 128.09182 & 18.63418 & 14.636 & 14.039 & 13.976 & 1086.0751610 \\
\hline KP400130 & 9.412 & 124.81942 & 14.45165 & 6.620 & 5.790 & 5.493 & 1044.0725050 \\
\hline KP400137 & 9.437 & 126.15084 & 14.65195 & 6.770 & 5.899 & 5.640 & 1046.0726780 \\
\hline KP400480 & 10.765 & 125.68731 & 16.52782 & 7.883 & 7.042 & 6.744 & 1065.0731300 \\
\hline KP400729 & 11.230 & 125.98279 & 21.18908 & 9.501 & 8.937 & 8.728 & 1111.0746480 \\
\hline KP400757 & 11.269 & 126.49398 & 20.24876 & 10.448 & 10.223 & 10.163 & 1102.0733020 \\
\hline KP400793 & 11.325 & 125.67915 & 19.44957 & 10.360 & 10.051 & 10.002 & 1094.0731010 \\
\hline KP400838 & 11.399 & 125.71576 & 16.03616 & 10.622 & 10.351 & 10.301 & 1060.0726140 \\
\hline KP400943 & 11.553 & 126.72200 & 21.46277 & 10.248 & 9.767 & 9.652 & 1114.0765910 \\
\hline KP400955 & 11.566 & 124.85908 & 17.83969 & 10.517 & 10.228 & 10.126 & 1078.0721630 \\
\hline KP401088 & 11.721 & 125.60951 & 20.98303 & 10.742 & 10.545 & 10.459 & 1109.0735130 \\
\hline KP401140 & 11.791 & 126.57688 & 23.25369 & 11.348 & 11.184 & 11.136 & 1132.0738260 \\
\hline
\end{tabular}

(cont'd) 
Table 4.1-Continued

\begin{tabular}{cccccccc}
\hline \hline $\begin{array}{c}\text { KELT } \\
\text { ID \# }\end{array}$ & $R_{K}$ & $\begin{array}{c}\text { RA } \\
(J 2000.0)\end{array}$ & $\begin{array}{c}\text { Dec } \\
(J 2000.0)\end{array}$ & & & & \\
KP401180 & 11.836 & 125.68698 & 19.46563 & 10.970 & 10.771 & 10.705 & 1094.0731050 \\
KP401189 & 11.845 & 126.57647 & 20.88048 & 11.134 & 10.925 & 10.859 & 1108.0737830 \\
KP401228 & 11.884 & 126.16900 & 18.70081 & 10.961 & 10.749 & 10.635 & 1087.0744760 \\
KP401417 & 12.065 & 124.71795 & 17.72634 & - & - & - & - \\
KP401692 & 12.282 & 124.87608 & 17.56528 & - & - & - & - \\
KP401909 & 12.450 & 125.93575 & 14.33630 & 11.870 & 11.659 & 11.599 & 1043.0725670 \\
KP401910 & 12.451 & 126.80627 & 17.67660 & 11.262 & 10.853 & 10.726 & 1076.0720650 \\
KP402009 & 12.510 & 126.62874 & 17.04807 & 11.173 & 10.712 & 10.598 & 1070.0731070 \\
KP402737 & 12.924 & 126.75819 & 18.81549 & 12.471 & 12.352 & 12.291 & 1088.0743890 \\
KP402860 & 12.978 & 125.85141 & 23.48793 & 12.271 & 12.009 & 11.960 & 1134.0730790 \\
KP402888 & 12.990 & 126.61546 & 23.15503 & - & - & - & - \\
KP404664 & 13.668 & 126.70888 & 16.38223 & 10.496 & 9.720 & 9.518 & 1063.0728570 \\
KP404675 & 13.670 & 125.72269 & 20.52251 & 12.953 & 12.608 & 12.538 & 1105.0736610 \\
KP404726 & 13.687 & 125.37290 & 20.86872 & 12.796 & 12.579 & 12.566 & 1108.0734050 \\
KP405535 & 13.923 & 126.53358 & 18.81866 & 13.382 & 13.041 & 12.940 & 1088.0743080 \\
KP407282 & 14.342 & 125.03220 & 21.74567 & 13.035 & 12.618 & 12.516 & 1117.0738510 \\
\hline
\end{tabular}

(cont'd) 
Table 4.1-Continued

\begin{tabular}{cccccccc}
\hline \hline $\begin{array}{c}\text { KELT } \\
\text { ID \# }\end{array}$ & $R_{K}$ & $\begin{array}{c}\text { RA } \\
(\mathrm{J} 2000.0)\end{array}$ & $\begin{array}{c}\text { Dec } \\
(\mathrm{J} 2000.0)\end{array}$ & & $H$ & $K$ & $\begin{array}{c}2 \mathrm{MASS}^{\mathrm{b}} \\
\text { ID \# }\end{array}$ \\
\hline $\mathrm{KP} 413347$ & 15.417 & 126.70529 & 20.46176 & 14.494 & 14.166 & 14.157 & 1104.0733930 \\
$\mathrm{KP} 414644$ & 15.648 & 125.77979 & 17.68299 & 14.464 & 14.020 & 13.919 & 1076.0717630 \\
\hline \hline
\end{tabular}

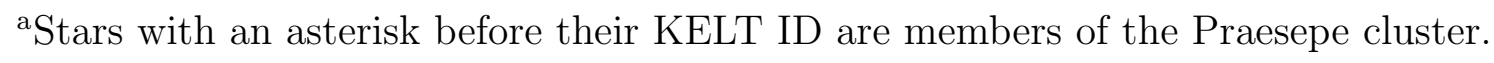

bStars with an asterisk after the 2MASS ID matched to more than one 2MASS source within the $99^{\prime \prime} 5$ matching radius. The 2MASS ID and $J H K$ colors in the table are for the closest match within the radius. 


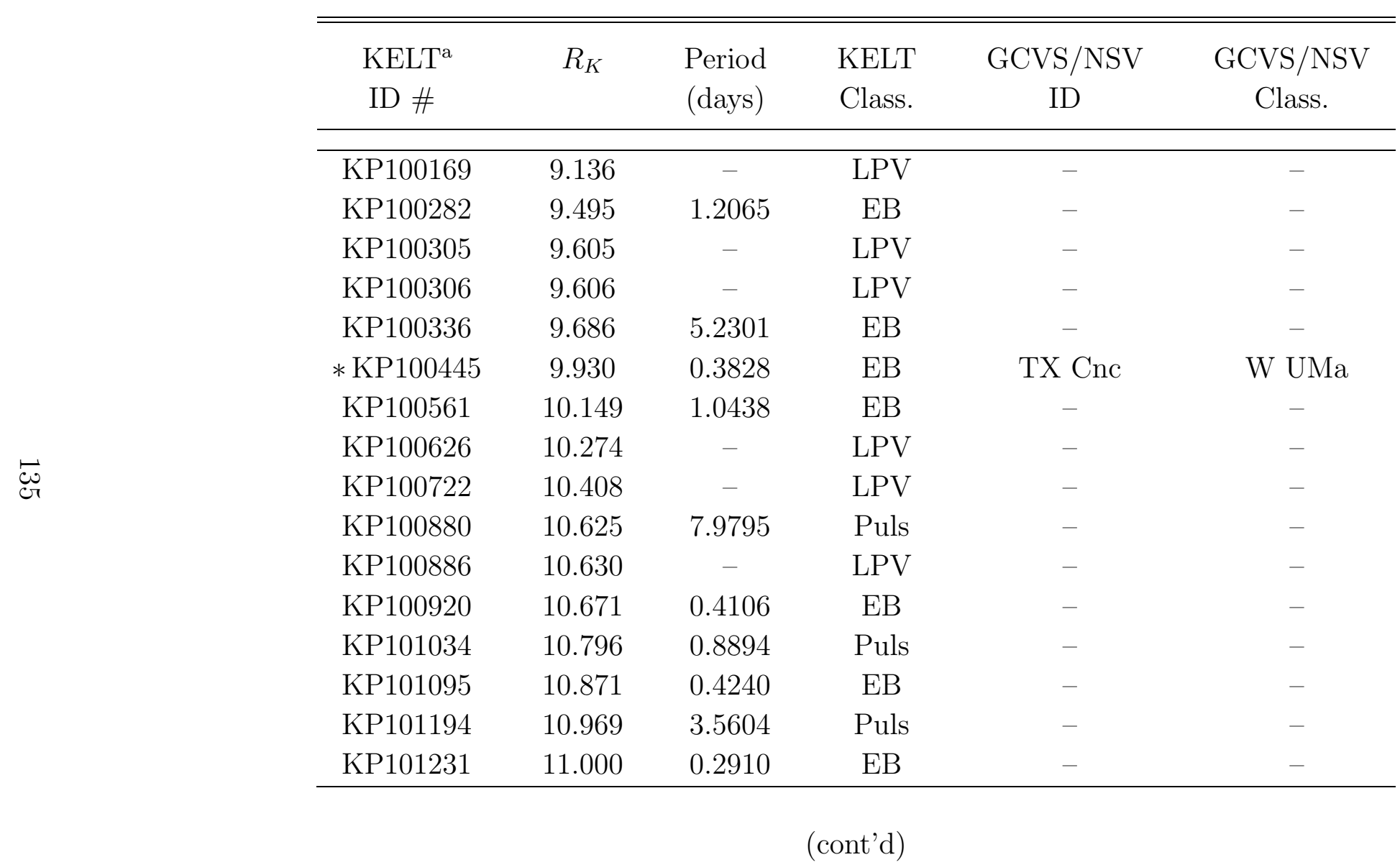

Table 4.2. Variable Stars Identified in KELT Observations of Praesepe: Variability 
Table 4.2-Continued

\begin{tabular}{|c|c|c|c|c|c|c|}
\hline & $\begin{array}{l}\text { KELT }^{\mathrm{a}} \\
\mathrm{ID} \#\end{array}$ & $R_{K}$ & $\begin{array}{l}\text { Period } \\
\text { (days) }\end{array}$ & $\begin{array}{l}\text { KELT } \\
\text { Class. }\end{array}$ & $\begin{array}{c}\text { GCVS/NSV } \\
\text { ID }\end{array}$ & $\begin{array}{c}\text { GCVS/NSV } \\
\text { Class. }\end{array}$ \\
\hline & KP101257 & 11.028 & 3.5740 & $\mathrm{~EB}$ & - & - \\
\hline & * KP101275 & 11.053 & 0.8067 & Puls & - & - \\
\hline & KP101456 & 11.209 & 0.4351 & Puls & - & - \\
\hline & KP101496 & 11.253 & - & LPV & - & - \\
\hline & KP101511 & 11.263 & 3.3393 & $\mathrm{~EB}$ & - & - \\
\hline & KP101549 & 11.289 & - & LPV & - & - \\
\hline & KP101674 & 11.402 & 0.1612 & Puls & - & - \\
\hline & KP101756 & 11.464 & 0.2958 & Puls & EF Cnc & W UMa \\
\hline & KP101831 & 11.509 & - & LPV & - & - \\
\hline & KP101896 & 11.552 & - & LPV & - & - \\
\hline & KP101921 & 11.573 & 0.5766 & $\mathrm{~EB}$ & - & - \\
\hline & KP102135 & 11.692 & 1.1039 & Puls & - & - \\
\hline & KP102245 & 11.749 & 0.5188 & $\mathrm{~EB}$ & - & - \\
\hline & KP102395 & 11.819 & - & LPV & - & - \\
\hline & KP102511 & 11.878 & 0.5584 & EB & - & - \\
\hline & KP102540 & 11.894 & 0.8213 & Puls & - & - \\
\hline
\end{tabular}


Table 4.2-Continued

\begin{tabular}{cccccc}
\hline \hline $\begin{array}{c}\text { KELT } \\
\text { ID \# }\end{array}$ & $R_{K}$ & $\begin{array}{c}\text { Period } \\
\text { (days) }\end{array}$ & $\begin{array}{c}\text { KELT } \\
\text { Class. }\end{array}$ & $\begin{array}{c}\text { GCVS/NSV } \\
\text { ID }\end{array}$ & $\begin{array}{c}\text { GCVS/NSV } \\
\text { Class. }\end{array}$ \\
\hline KP102588 & 11.916 & 1.3244 & EB & NSV 04269 & V \\
KP102705 & 11.980 & 0.5978 & Puls & - & - \\
KP102807 & 12.030 & 0.8599 & Puls & - & - \\
KP102811 & 12.034 & 0.6102 & EB & - & - \\
KP102836 & 12.047 & 3.1742 & EB & - & - \\
KP102908 & 12.082 & - & LPV & - & - \\
KP102979 & 12.112 & 0.8773 & Puls & - & - \\
KP103010 & 12.124 & 1.2578 & EB & - & - \\
$*$ KP103073 & 12.150 & 0.8246 & Puls & - & RR Lyr \\
KP103143 & 12.176 & 1.2542 & EB & - & - \\
KP103254 & 12.226 & 0.5458 & Puls & EZ Cnc & - \\
KP103267 & 12.229 & - & LPV & - & - \\
KP103271 & 12.232 & 0.8943 & Puls & - & - \\
KP103285 & 12.237 & 0.3550 & EB & - & - \\
KP103393 & 12.280 & 0.5763 & EB & - & - \\
KP103585 & 12.345 & 4.4082 & EB & & - \\
\hline
\end{tabular}

(cont'd) 
Table 4.2-Continued

\begin{tabular}{cccccc}
\hline \hline $\begin{array}{c}\text { KELT } \\
\text { ID \# }\end{array}$ & $R_{K}$ & $\begin{array}{c}\text { Period } \\
\text { (days) }\end{array}$ & $\begin{array}{c}\text { KELT } \\
\text { Class. }\end{array}$ & $\begin{array}{c}\text { GCVS/NSV } \\
\text { ID }\end{array}$ & $\begin{array}{c}\text { GCVS/NSV } \\
\text { Class. }\end{array}$ \\
\hline KP103593 & 12.348 & 0.3536 & EB & - & - \\
KP103608 & 12.353 & 8.8821 & Puls & - & - \\
KP103772 & 12.410 & 0.2603 & EB & - & - \\
KP104115 & 12.530 & 2.6984 & EB & - & - \\
KP104166 & 12.549 & - & LPV & - & - \\
KP104174 & 12.552 & 0.3637 & EB & NSV 04207 & V UMa \\
KP104185 & 12.557 & 0.2814 & EB & GW Cnc & - \\
KP104317 & 12.599 & 0.3464 & EB & - & - \\
KP104332 & 12.602 & 0.2698 & EB & - & - \\
KP104424 & 12.629 & - & LPV & - & - \\
KP104475 & 12.644 & 2.4236 & Puls & - & - \\
KP104572 & 12.670 & 0.0865 & Puls & - & - \\
KP104597 & 12.676 & 0.4586 & EB & - & - \\
KP104630 & 12.683 & - & LPV & - & EA/SD \\
KP104850 & 12.741 & - & LPV & - & - \\
KP104855 & 12.742 & 1.0929 & EB & RY Cnc & \\
\hline
\end{tabular}

(cont'd) 
Table 4.2-Continued

\begin{tabular}{|c|c|c|c|c|c|}
\hline $\begin{array}{l}\text { KELT }^{\mathrm{a}} \\
\text { ID \# }\end{array}$ & $R_{K}$ & $\begin{array}{l}\text { Period } \\
\text { (days) }\end{array}$ & $\begin{array}{l}\text { KELT } \\
\text { Class. }\end{array}$ & $\begin{array}{c}\text { GCVS/NSV } \\
\text { ID }\end{array}$ & $\begin{array}{c}\text { GCVS/NSV } \\
\text { Class. }\end{array}$ \\
\hline KP104917 & 12.760 & 0.7091 & EB & - & - \\
\hline KP105076 & 12.804 & 0.1489 & Puls & - & - \\
\hline KP105119 & 12.815 & 0.7477 & EB & - & - \\
\hline KP105369 & 12.877 & 11.0424 & Puls & - & - \\
\hline KP105756 & 12.971 & - & LPV & - & - \\
\hline KP105772 & 12.975 & - & LPV & - & - \\
\hline KP105793 & 12.979 & 0.3216 & $\mathrm{~EB}$ & - & - \\
\hline KP105899 & 13.005 & 0.8162 & Puls & - & - \\
\hline KP106106 & 13.054 & 0.6997 & EB & - & - \\
\hline KP106218 & 13.077 & - & LPV & - & - \\
\hline KP106227 & 13.080 & - & LPV & - & - \\
\hline KP106319 & 13.096 & 0.1846 & Puls & - & - \\
\hline KP106351 & 13.102 & 0.5430 & Puls & AN Cnc & RR Lyr \\
\hline KP106452 & 13.122 & 0.5246 & Puls & CQ Cnc & RR Lyr \\
\hline KP106608 & 13.157 & 0.3396 & EB & - & - \\
\hline KP106885 & 13.213 & - & LPV & - & - \\
\hline
\end{tabular}

(cont'd) 
Table 4.2-Continued

\begin{tabular}{cccccc}
\hline \hline $\begin{array}{c}\text { KELT } \\
\text { ID \# }\end{array}$ & $R_{K}$ & $\begin{array}{c}\text { Period } \\
\text { (days) }\end{array}$ & $\begin{array}{c}\text { KELT } \\
\text { Class. }\end{array}$ & $\begin{array}{c}\text { GCVS/NSV } \\
\text { ID }\end{array}$ & $\begin{array}{c}\text { GCVS/NSV } \\
\text { Class. }\end{array}$ \\
\hline KP107014 & 13.234 & 0.2465 & Puls & - & - \\
KP107531 & 13.333 & 0.5516 & EB & - & - \\
KP107924 & 13.408 & 0.5268 & EB & - & - \\
KP108285 & 13.473 & 0.3360 & EB & - & - \\
KP108858 & 13.569 & 0.2986 & EB & - & - \\
KP109198 & 13.619 & 1.7433 & EB & - & - \\
KP109247 & 13.625 & 0.4205 & EB & - & - \\
KP110021 & 13.734 & 0.3860 & EB & - & - \\
KP110124 & 13.751 & 0.0576 & Puls & - & - \\
KP110177 & 13.760 & 0.4420 & EB & - & - \\
KP110305 & 13.777 & 0.2719 & EB & - & - \\
KP110392 & 13.792 & 0.6004 & Puls & - & - \\
KP110617 & 13.826 & 0.3395 & EB & - & - \\
KP110674 & 13.837 & 11.0384 & EB & - & - \\
KP110871 & 13.864 & 0.2836 & EB & - & - \\
KP110876 & 13.865 & 0.3013 & EB & & - \\
\hline
\end{tabular}

(cont'd) 
Table 4.2-Continued

\begin{tabular}{cccccc}
\hline \hline $\begin{array}{c}\text { KELT } \\
\text { ID \# }\end{array}$ & $R_{K}$ & $\begin{array}{c}\text { Period } \\
\text { (days) }\end{array}$ & $\begin{array}{c}\text { KELT } \\
\text { Class. }\end{array}$ & $\begin{array}{c}\text { GCVS/NSV } \\
\text { ID }\end{array}$ & $\begin{array}{c}\text { GCVS/NSV } \\
\text { Class. }\end{array}$ \\
\hline KP111363 & 13.933 & - & LPV & - & - \\
KP112203 & 14.042 & 0.3567 & EB & - & - \\
KP112538 & 14.086 & 0.2722 & EB & - & - \\
KP113453 & 14.197 & 0.3395 & Puls & - & - \\
KP113808 & 14.236 & 0.3182 & EB & - & - \\
KP114383 & 14.301 & 0.3478 & EB & - & - \\
KP114757 & 14.344 & - & LPV & - & - \\
KP115386 & 14.408 & 0.2981 & Puls & - & - \\
KP115639 & 14.434 & 0.5692 & EB & - & - \\
KP115973 & 14.469 & - & LPV & - & - \\
KP118312 & 14.698 & - & LPV & - & - \\
KP118899 & 14.754 & 0.2897 & EB & - & - \\
KP119499 & 14.808 & - & LPV & - & - \\
KP121436 & 14.975 & - & LPV & - & - \\
KP126266 & 15.351 & - & LPV & - & - \\
KP127604 & 15.455 & - & LPV & & - \\
\hline
\end{tabular}

(cont'd) 
Table 4.2-Continued

\begin{tabular}{|c|c|c|c|c|c|}
\hline $\begin{array}{l}\text { KELT }^{\mathrm{a}} \\
\mathrm{ID} \#\end{array}$ & $R_{K}$ & $\begin{array}{l}\text { Period } \\
\text { (days) }\end{array}$ & $\begin{array}{l}\text { KELT } \\
\text { Class. }\end{array}$ & $\begin{array}{c}\text { GCVS/NSV } \\
\text { ID }\end{array}$ & $\begin{array}{c}\text { GCVS/NSV } \\
\text { Class. }\end{array}$ \\
\hline KP127745 & 15.466 & - & LPV & - & - \\
\hline KP128381 & 15.511 & - & LPV & - & - \\
\hline KP130733 & 15.689 & - & LPV & - & - \\
\hline KP131942 & 15.791 & - & LPV & - & - \\
\hline KP133934 & 16.024 & - & LPV & - & - \\
\hline KP200074 & 9.079 & - & LPV & - & - \\
\hline KP200152 & 9.711 & - & LPV & - & - \\
\hline KP200188 & 10.019 & 10.0591 & $\mathrm{~EB}$ & RU Cnc & $\mathrm{EA} / \mathrm{DS} / \mathrm{RS}$ \\
\hline KP200191 & 10.024 & - & LPV & - & - \\
\hline KP200210 & 10.116 & - & LPV & - & - \\
\hline KP200224 & 10.184 & 12.1114 & Puls & - & - \\
\hline KP200244 & 10.312 & - & LPV & - & - \\
\hline KP200257 & 10.384 & - & LPV & - & - \\
\hline KP200262 & 10.408 & - & LPV & - & - \\
\hline KP200270 & 10.453 & 0.7277 & $\mathrm{~EB}$ & - & - \\
\hline KP200312 & 10.600 & 2.1302 & $\mathrm{~EB}$ & - & - \\
\hline
\end{tabular}


Table 4.2-Continued

\begin{tabular}{|c|c|c|c|c|c|c|}
\hline & $\begin{array}{l}\text { KELT }^{\mathrm{a}} \\
\mathrm{ID} \#\end{array}$ & $R_{K}$ & $\begin{array}{l}\text { Period } \\
\text { (days) }\end{array}$ & $\begin{array}{l}\text { KELT } \\
\text { Class. }\end{array}$ & $\begin{array}{c}\text { GCVS/NSV } \\
\text { ID }\end{array}$ & $\begin{array}{c}\text { GCVS/NSV } \\
\text { Class. }\end{array}$ \\
\hline & KP200490 & 11.146 & 0.1297 & Puls & - & - \\
\hline & KP200596 & 11.385 & 0.3234 & $\mathrm{~EB}$ & - & - \\
\hline & KP200643 & 11.489 & 0.3866 & $\mathrm{~EB}$ & - & - \\
\hline & KP200711 & 11.642 & 0.2609 & Puls & - & - \\
\hline & KP200738 & 11.701 & 0.4046 & $\mathrm{~EB}$ & - & - \\
\hline & * KP200757 & 11.745 & 1.1150 & Puls & - & - \\
\hline & KP201187 & 12.361 & 1.4783 & $\mathrm{~EB}$ & - & - \\
\hline$\vec{c}$ & KP201350 & 12.543 & 0.8728 & $\mathrm{~EB}$ & - & - \\
\hline & KP201399 & 12.596 & 0.2163 & Puls & - & - \\
\hline & KP201426 & 12.636 & 1.5944 & $\mathrm{~EB}$ & - & - \\
\hline & KP201594 & 12.800 & - & $\mathrm{LPV}$ & - & - \\
\hline & KP201632 & 12.837 & 0.3361 & $\mathrm{~EB}$ & - & - \\
\hline & KP202047 & 13.178 & 0.6916 & $\mathrm{~EB}$ & - & - \\
\hline & KP202125 & 13.224 & 0.3682 & $\mathrm{~EB}$ & - & - \\
\hline & KP202179 & 13.269 & 0.6204 & $\mathrm{~EB}$ & - & - \\
\hline & KP202440 & 13.435 & 2.7325 & $\mathrm{~EB}$ & - & - \\
\hline
\end{tabular}


Table 4.2-Continued

\begin{tabular}{|c|c|c|c|c|c|c|}
\hline & $\begin{array}{l}\text { KELT }^{\mathrm{a}} \\
\mathrm{ID} \#\end{array}$ & $R_{K}$ & $\begin{array}{l}\text { Period } \\
\text { (days) }\end{array}$ & $\begin{array}{l}\text { KELT } \\
\text { Class. }\end{array}$ & $\begin{array}{c}\text { GCVS/NSV } \\
\text { ID }\end{array}$ & $\begin{array}{c}\text { GCVS/NSV } \\
\text { Class. }\end{array}$ \\
\hline \multirow{16}{*}{$\vec{E}$} & KP202483 & 13.459 & - & LPV & - & - \\
\hline & KP202655 & 13.556 & 0.3784 & $\mathrm{~EB}$ & NSV 04158 & $\mathrm{~V}$ \\
\hline & KP202778 & 13.608 & 1.3931 & $\mathrm{~EB}$ & - & - \\
\hline & KP202994 & 13.717 & - & LPV & - & - \\
\hline & KP203288 & 13.858 & 0.5163 & EB & - & - \\
\hline & KP204370 & 14.282 & 0.1534 & Puls & - & - \\
\hline & KP204605 & 14.381 & 0.1667 & Puls & - & - \\
\hline & KP206403 & 14.927 & 0.2525 & $\mathrm{~EB}$ & - & - \\
\hline & KP300063 & 9.219 & - & LPV & - & - \\
\hline & KP300133 & 9.785 & 0.1089 & Puls & - & - \\
\hline & KP300135 & 9.788 & 0.8270 & Puls & FR Cnc & BY Dra \\
\hline & KP300161 & 9.982 & 1.4661 & $\mathrm{~EB}$ & - & - \\
\hline & KP300277 & 10.581 & 1.3236 & $\mathrm{~EB}$ & FF Cnc & Algol \\
\hline & KP300434 & 11.088 & - & LPV & - & - \\
\hline & KP300526 & 11.290 & 0.3154 & EB & - & - \\
\hline & KP300603 & 11.482 & - & LPV & GV Cnc & $\mathrm{SR}$ \\
\hline
\end{tabular}


Table 4.2-Continued

\begin{tabular}{cccccc}
\hline \hline $\begin{array}{c}\text { KELT } \\
\text { ID \# }\end{array}$ & $R_{K}$ & $\begin{array}{c}\text { Period } \\
\text { (days) }\end{array}$ & $\begin{array}{c}\text { KELT } \\
\text { Class. }\end{array}$ & $\begin{array}{c}\text { GCVS/NSV } \\
\text { ID }\end{array}$ & $\begin{array}{c}\text { GCVS/NSV } \\
\text { Class. }\end{array}$ \\
\hline KP300608 & 11.496 & 7.6757 & Puls & - & - \\
KP300656 & 11.603 & 2.2290 & EB & - & - \\
KP300678 & 11.658 & - & LPV & - & - \\
KP300976 & 12.169 & - & LPV & - & - \\
KP301090 & 12.320 & 0.8230 & Puls & - & - \\
KP301148 & 12.393 & 0.3113 & EB & - & - \\
KP301208 & 12.453 & 0.2175 & Puls & - & - \\
KP301312 & 12.545 & 0.8214 & EB & - & - \\
KP301510 & 12.736 & 0.1840 & Puls & - & - \\
KP301583 & 12.805 & 0.3393 & EB & - & - \\
KP301835 & 13.020 & 0.2393 & EB & - & - \\
KP301980 & 13.128 & 0.1390 & Puls & - & - \\
KP302158 & 13.233 & 0.2626 & EB & - & - \\
KP302288 & 13.300 & 0.5335 & EB & - & - \\
KP302426 & 13.382 & 0.2975 & EB & - & - \\
KP302468 & 13.405 & 0.2249 & Puls & & - \\
\hline
\end{tabular}

(cont'd) 
Table 4.2-Continued

\begin{tabular}{cccccc}
\hline \hline $\begin{array}{c}\text { KELT } \\
\text { ID \# }\end{array}$ & $R_{K}$ & $\begin{array}{c}\text { Period } \\
\text { (days) }\end{array}$ & $\begin{array}{c}\text { KELT } \\
\text { Class. }\end{array}$ & $\begin{array}{c}\text { GCVS/NSV } \\
\text { ID }\end{array}$ & $\begin{array}{c}\text { GCVS/NSV } \\
\text { Class. }\end{array}$ \\
\hline KP302519 & 13.437 & 2.2423 & EB & - & - \\
KP302538 & 13.448 & 0.3481 & EB & - & - \\
KP303144 & 13.747 & 0.4900 & Puls & - & - \\
KP303492 & 13.890 & - & LPV & - & - \\
KP309546 & 15.612 & 0.1089 & Puls & - & - \\
KP400130 & 9.412 & - & LPV & - & - \\
KP400137 & 9.437 & - & LPV & - & - \\
KP400480 & 10.765 & - & LPV & - & - \\
KP400729 & 11.230 & 7.8954 & Puls & - & - \\
KP400757 & 11.269 & 0.9744 & EB & - & - \\
KP400793 & 11.325 & 0.2800 & EB & - & - \\
KP400838 & 11.399 & 0.8538 & EB & - & - \\
KP400943 & 11.553 & 0.6944 & Puls & - & - \\
KP400955 & 11.566 & 0.7844 & EB & - & V \\
KP401088 & 11.721 & 0.5609 & EB & - & - \\
KP401140 & 11.791 & 0.7178 & EB & NSV 04069 & - \\
\hline
\end{tabular}

(cont'd) 
Table 4.2-Continued

\begin{tabular}{cccccc}
\hline \hline $\begin{array}{c}\text { KELT } \\
\text { ID \# }\end{array}$ & $R_{K}$ & $\begin{array}{c}\text { Period } \\
\text { (days) }\end{array}$ & $\begin{array}{c}\text { KELT } \\
\text { Class. }\end{array}$ & $\begin{array}{c}\text { GCVS/NSV } \\
\text { ID }\end{array}$ & $\begin{array}{c}\text { GCVS/NSV } \\
\text { Class. }\end{array}$ \\
\hline KP401180 & 11.836 & 0.3257 & EB & - & - \\
KP401189 & 11.845 & 0.4180 & EB & EH Cnc & W UMa \\
KP401228 & 11.884 & 1.7831 & EB & - & - \\
KP401417 & 12.065 & 0.7835 & EB & - & - \\
KP401692 & 12.282 & 1.2360 & EB & - & - \\
KP401909 & 12.450 & 0.3358 & EB & - & - \\
KP401910 & 12.451 & 3.2616 & EB & - & - \\
KP402009 & 12.510 & 0.2671 & EB & - & - \\
KP402737 & 12.924 & 0.8880 & EB & - & - \\
KP402860 & 12.978 & 0.5166 & EB & - & - \\
KP402888 & 12.990 & 0.9017 & EB & - & - \\
KP404664 & 13.668 & - & LPV & - & - \\
KP404675 & 13.670 & 0.3067 & EB & - & - \\
KP404726 & 13.687 & 0.3759 & EB & - & - \\
KP405535 & 13.923 & - & LPV & - & - \\
KP407282 & 14.342 & 0.3051 & EB & & - \\
\hline
\end{tabular}

(cont'd) 
Table 4.2 - Continued

\begin{tabular}{cccccc}
\hline \hline $\begin{array}{c}\text { KELT } \\
\text { ID } \#\end{array}$ & $R_{K}$ & $\begin{array}{c}\text { Period } \\
\text { (days) }\end{array}$ & $\begin{array}{c}\text { KELT } \\
\text { Class. }\end{array}$ & $\begin{array}{c}\text { GCVS/NSV } \\
\text { ID }\end{array}$ & $\begin{array}{c}\text { GCVS/NSV } \\
\text { Class. }\end{array}$ \\
\hline KP413347 & 15.417 & - & LPV & - & - \\
KP414644 & 15.648 & - & LPV & - & - \\
\hline \hline
\end{tabular}

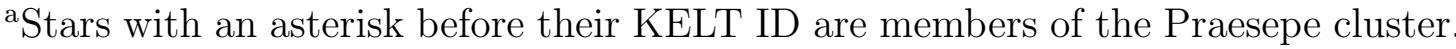




\section{Chapter 5}

\section{Discussion}

\subsection{Results of Testing and Commissioning Phases}

The analysis of the KELT observations of the Praesepe field from Chapter 4 shows that KELT is able to detect astrophysical variation at the level of transit signals. We have derived lightcurves for hundreds of stars with RMS variations of less than $1 \%$ and thousands of stars with $\mathrm{RMS}<2 \%$. We are able to remove systematic trends (§4.5.1) and find lightcurves with transit-like signals (§4.7). The fact that subsequent observations prove that such signals are real demonstrates that KELT has the necessary capabilities to detect planetary transits.

The upcoming analysis of the KELT survey data will be the core of the KELT experiment. The commissioning data has been an invaluable testbed for the design of the reduction process and has yielded significant science already with newly discovered variable stars. The results of that work (Chapter 4) will be submitted for publication soon. 


\subsection{MAIN KELT SURVEY}

The primary mission of the KELT project is to conduct a multi-year survey across a large portion of the sky to detect planetary transits, based on the parameters established in Chapter 2. The survey utilizes the $80 \mathrm{~mm}$ lens described in Chapter 3, and has been underway since late 2004, with large gaps in operations for a variety of reasons. Every summer the weather in Arizona becomes cloudy and rainy, so Winer Observatory ceases all operations and turns off most equipment during that time, which usually lasts from July through mid-September. In addition to those breaks, KELT was shut down from 2005 November 19 through 2006 April 23 because the camera broke and was returned to the manufacturer for repair. Additionally, the survey was suspended from February through April 2005 for the Praesepe campaign. The full campaign is planned to continue through summer 2008, with a break for the summer 2007 rainy season.

While the scenarios considered in Chapter 1 assumed a survey covering the entire sky, the actual KELT survey is more limited in coverage. With a single telescope only one hemisphere is accessible, and it can be difficult to maintain regular coverage of regions far to the North or South. We therefore selected for the KELT survey to cover a strip entirely around the sky broken into separate equally spaced fields the width of the KELT field of view with the $80 \mathrm{~mm}$ camera, $26^{\circ} \times 26^{\circ}$. The fields are all at the Declination equal to the latitude of Winer Observatory, 
and adjacent fields overlap by about $3^{\circ}$. The locations of the fields are portrayed in schematic in Figure 5.1 .

The survey observing strategy consists of tiling between multiple fields throughout the night, as described in §3.2.3. From 2004 through summer 2006 an exposure time of 30 seconds was used for all images. When observing started after the summer shutdown in September that year, the exposure time was increased to 150 seconds to probe fainter stars and to reduce the fraction of time spent on readout.

Another change that was implemented partway through the survey was a slight defocusing of the camera after the camera was fixed and reinstalled in April 2005. This change was made to reduce the undersampling of the PSFs of the stars on the images, and increased the mean FWHM of the PSFs from about $\sim 2.2$ pixels to $\sim 3.1$ pixels.

One more parameter that has changed since the beginning of the survey has been the algorithm used to choose the series of fields to observe each night. Through summer of 2006, the telescope observed a small number of fields across the whole night. Starting after the summer 2006 shutdown, we adjusted the strategy to observe more fields fewer times during the night. This change spreads out the observations more consistently across the different fields. 


\subsection{Current Status of KeLt and Future Work}

KELT has now been in operation for well over two years, and has taken nearly 40,000 images for the primary survey. An illustration of the observational record is shown in Figure 5.2, where the number of images observed per night is plotted over the course of the survey for each field. Periods when the telescope was shut down are shaded, and the total number of images are listed on the right.

We have begun the process of reducing the survey data - a significant undertaking given the number of images and the size of the disk space needed to contain them. The analysis itself requires significant bookkeeping efforts and computer processor time, and we anticipate that the initial phases should take a few months. Since the summer 2007 shutdown is approaching, we hope to have initial results by the time the observing season starts again in September. We will be searching the lightcurves for transits using the methods described in Chapter 4 , along with more sophisticated transit selection algorithms more appropriate for large numbers of lightcurves. The telescope will continue to operate for the foreseeable future, developing long time baseline lightcurves that will enhance the transit detection potential of the data set.

There are also other potential scientific uses for the KELT data. The discovery of previously unknown variable stars from the KELT commissioning data is a clear example of how transit surveys are well positioned to find and characterize variable 
stars. In addition, the archived data will permit searching for any transient source within the magnitude range probed by KELT. Such phenomena could be near-Earth asteroids, bright gamma-ray-burst afterglows, or supernovae. 


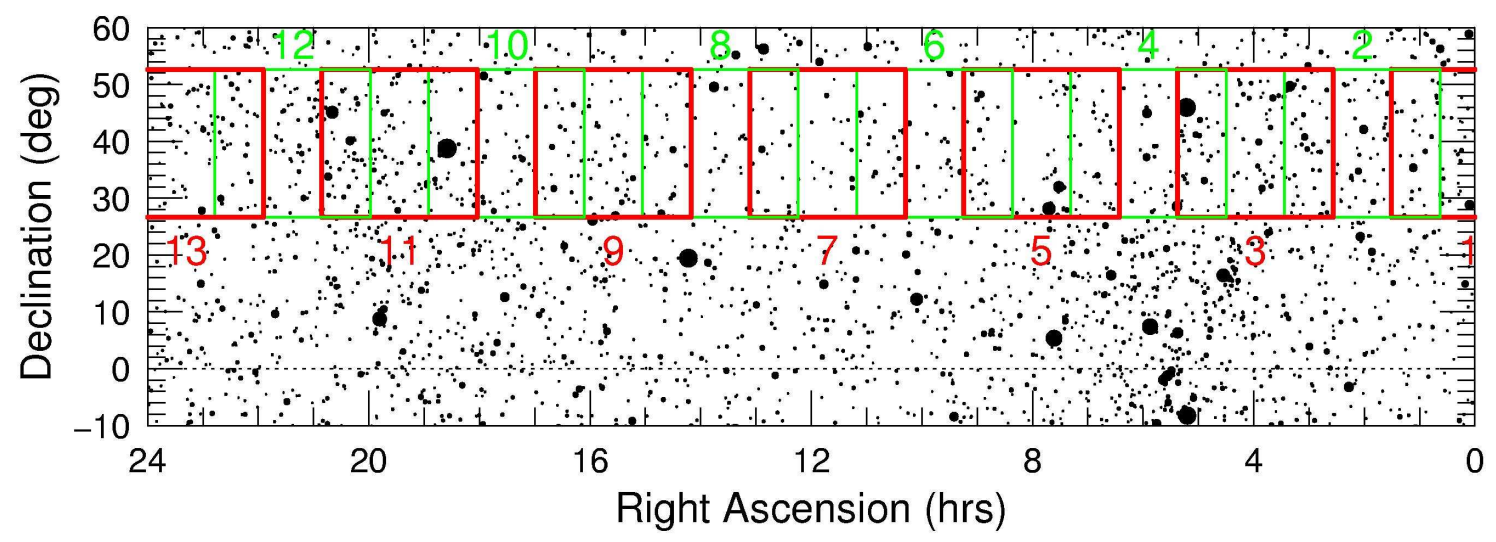

Fig. 5.1. - Schematic map of the thirteen KELT survey fields. 


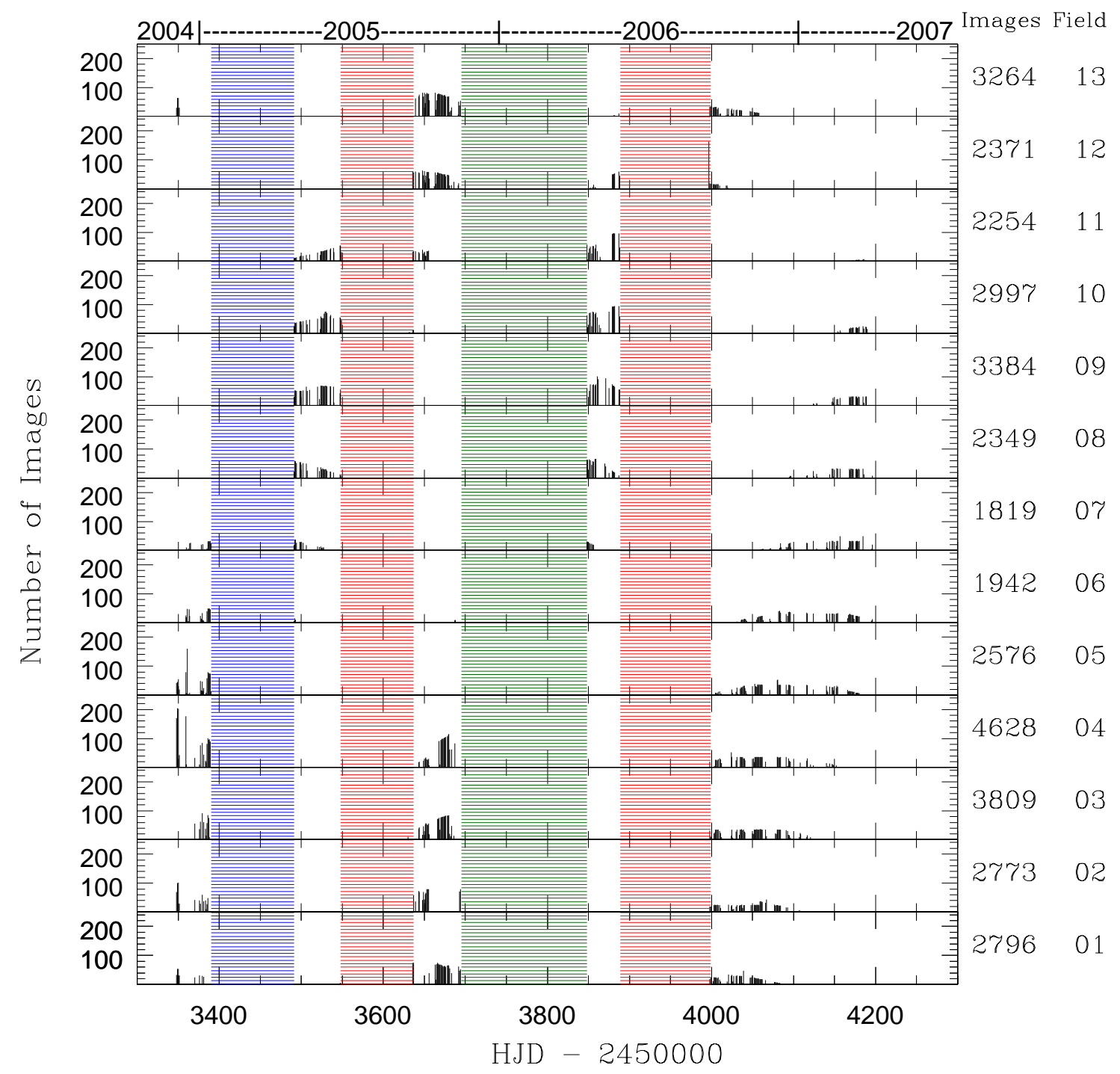

Fig. 5.2.- The distribution of the KELT survey observations per night plotted across time for each of the survey fields. Time runs from December 2004 through March 2007. Data taken since 2007 April 6 has not gone through initial processing and does not appear on the plot. All data taken before summer 2006 has been filtered for poor quality images based on sky-level and FWHM. Red shaded regions indicate summer shutdown of Winer Observatory. The blue region shows the period when the Praesepe campaign took place, and the green region shows the time when the camera broke and was being repaired. 


\section{BIBLIOGRAPHY}

Adams, J. D., Stauffer, J. R., Skrutskie, M. F., Monet, D. G., Portegies Zwart, S. F., Janes, K. A., \& Beichman, C. A. 2002, AJ, 124, 1570

Alard, C. \& Lupton, R.H. 1998, ApJ, 503, 325

Alard, C. 2000, A\&A, 144, 363

Alonso, R., et al. 2004, ApJ, 613, L153

An, D., Terndrup, D. M., Pinsonneault, M. H., Paulson, D. B., Hanson, R. B., \& Stauffer, J. R. 2007, ApJ, 655, 233

Bakos, G., Noyes, R. W., Kovacs, G., Stanek, K. Z., Sasselov, D. D., \& Domsa, I. 2004, PASP, 116, 266

Bakos, G. A. 2006, private communication

Bakos, G. A. et al. 2007a, ApJ, 656, 552

Bakos, G. A. et al. 2007b, submitted to ApJ, astro-ph/0705.0126

Barnes, J. W. \& Fortnoy, J. J. 2004, ApJ, 616, 1193

Bessell M. S., \& Brett J. M. 1988, PASP, 100, 1134

Bessell M. S., \& Stringfellow G. S. 1993, ARA\&A, 31, 433

Binney, J. \& Merrifield, M. 1998, Galactic Astronomy (Princeton: Princeton University Press)

Bouchy, F. 2005, A \& A, 444, L15

Brown, T. M. 2003, ApJ, 593, L125

Burke, C. J., DePoy, D. L., Gaudi, B. S., Marshall, J. L., \& Pogge, R. W. 2002, in ASP Conf. Ser. 294: Scientific Frontiers in Research on Extrasolar Planets, eds. D. Deming and S. Seager (ASP: San Francisco), 379 
Burke, C. J., Gaudi, B. S., DePoy, D. L., \& Pogge, R. W. 2006, AJ, 132, 210

Burke, C. J., et al. 2007, submitted to ApJ, astro-ph/0705.0003

Calabretta, M.R., \& Greisen, E.W. 2002, å, 395, 1077

Cameron, A. C., et al. 2007, MNRAS, 375, 951

Chappelle, R. J., Pinfield, D. J., Steele, I. A., Dobbie, P. D., \& Magazzu, A. 2005, MNRAS, 361, 1323

Charbonneau, D., Brown, T. M., Latham, D. W., \& Mayor, M. 2000, ApJ, 529, L45

Charbonneau, D., Brown, T. M., Noyes, R. W., \& Gilliland R. L. 2002, ApJ, 568, 377

Charbonneau, D., Brown, T. M., Burrows, A., \& Laughlin, G. 2007, in Protostars \& Planets V, ed. B. Reipurth, D. Jewitt, \& K. Keil (Tucson: University of Arizona Press), 701

Cody, A. M., \& Sasselov, D. D. 2002, ApJ, 569, 451

European Space Agency (ESA). 1997, The Hipparcos and Tycho Catalogues (SP-1200; Noordwijk: ESA)

Everett, M. E. \& Howell, S. B. 2001, PASP, 113, 1428

Gaudi B. S. \& Winn, J. N. 2007, ApJ, 655, 550

Gould, A. \& Morgan, C. W. 2003, ApJ, 585, 1056

Guillot, T. 2005, Annual Review of Earth and Planetary Sciences, 33, 493

Hartman, J. D., Bakos, G., Stanek, K. Z., \& Noyes, R. W. 2004, AJ, 128, 1761

Hayes, D. S. \& Latham, D. W. 1975, ApJ, 197, 593

Henry, G. W., Marcy, G. W., Butler, R. P., \& Vogt, S. S. 2000, ApJ, 529, L41

Høg, E., et al. 2000, A\&A, 355, L27

Howell, S. B., Everett, M., Davis, D. R., Weidenschilling, S. J., McGruder, C. H., III, \& Gelderman, R. 2000, BAAS, 32, 3203

Hui, L., \& Seager, S. 2002, ApJ, 572, 540

Ivezic, Z., et al. 2003, Mem. Soc. Astron. Ital., 20, 1

Jones, B. F. \& Stauffer, J. R. 1991, AJ, 102, 1080 
Kaluzny, J., Stanek, K. Z., Krockenberger, M., Sasselov, D. D., Tonry, J. L., \& Mateo, M. 1998, AJ, 115, 1016

Kodak Photographic Filters Handbook, Publication No. B-3 Eastman Kodak Company, Rochester, New York (1998)

Konacki, M., Torres, G., Jha, S., and Sasselov, D. D. 2003, Nature 421, 507

Kovacs, G., Zucker, S., \& Mazeh, T. 2002, å, 391, 369

Kukarkin, B. V., et al. 1982, New Catalog of Suspected Variable Stars (Moscow: Nauka)

Latham, D. 2007, private communication

Mallen-Ornelas, G., Seager, S., Yee, H. K. C., Minniti, D., Gladders, M. D., Ellison, S., Brown, T., \& Mallen, G. M. 2001, BAAS, 199, 6602

Mayor, M. \& Queloz, D. 1995, Nature, 378, 355

Mazeh, T., et al. 2000, ApJ, 532, L55

Mazeh, T., Tamuz, O., \& Zucker, S. 2006, astro-ph/0612418

McCullough, P. R., Stys, J. E., Valenti, J. A., Fleming, S. W., Janes, K. A., \& Heasley, J. N. 2005, PASP, 117, 783

McCullough, P. R., et al. 2006, ApJ, 648, 1228

Meisenzahl, E., Chang, W., DesJardin, W., Kosman, S., Shepherd, J., Stevens, E., \& Wong, K. 2000, Proc. SPIE, 3965, 92

O’Donovan, F. T., et al. 2006, ApJ, 651L, 61

Pepper, J., Gould, A., \& Depoy, D. L., 2003, Acta Astron, 53, 213

Pojmanski, G. 2000, AcA, 50, 177

Pollacco, D. L., et al. 2006, PASP, 118, 1407

Pont, F., Zuker, S., \& Queloz, D. 2006, MNRAS, 373, 231

Reid, N. 1991, AJ, 102, 1428

Rosenblatt, F. 1971, Icarus, 14, 71

Samus, N. N. \& Durlevich, O. V. 2004, Combined General Catalog of Variable Stars (ed. 4.2; Moscow: Sternberg Astron. Inst.) 
Sato, B. et al. 2005, ApJ, 633, 465

Schlegel, D. J. Finkbeiner, D. P., Davis, M. 1998, ApJ, 500, 525

Schneider, D. P., Gunn, J. E., \& Hoessel, J.G. 1983, ApJ, 264, 337

Schwarzenberg-Czerny, A. 1996, ApJ, 460, L107

Skrutskie, M. F. 2006, AJ, 131, 1163

Stetson, P. B. 1987, PASP, 99, 191

Stetson, P. B. 1996, PASP, 108, 851

Street, R. A. et al. 2000, in ASP Conf. Ser., Vol. 219, Euroconference on Disks, Planetesimals and Planets, eds. F. Garzon, C. Eiroa, D. de Winter, \& T. J. Mahoney (San Francisco: ASP), 572

Tamuz, O., Mazeh, T., \& Zuker, S. 2005, MNRAS, 356, 1466

Udalski, A. et al. 2002, AcA, 52, 1

Udalski, A., Zebrun, K., Szymanski, M., Kubiak, M., Soszynski, I., Szewczyk, O., Wyrzykowski, L., Pietrzynski, G. 2002, Acta Astron, 52, 1

Udalski, A., Szewczyk, O., Zebrun, K., Pietrzynski, G., Szymanski, M., Kubiak, M., Soszynski, I., Wyrzykowski, L. 2002, Acta Astron, 52, 317

Udalski, A., Pietrzynski, G., Szymanski, M., Kubiak, M., Zebrun, K., Soszynski, I., Szewczyk, O., Wyrzykowski, L. 2003, Acta Astron, 53, 133

Udalski, A., Szymanski, M. K., Kubiak, M., Pietrzynski, G., Soszynski, I., Zebrun, K., Szewczyk, O., \& Wyrzykowski, L. 2004, Acta, 54, 313

van Belle, G. T. 1999, PASP, 111, 1515

Wolszczan, A., \& Frail, D. A. 1992, Nature, 355, 145

Warner, B. 1988, High Speed Astronomical Photometry (Cambridge, UK: Cambridge University Press)

Young, A. T. 1967, AJ, 72, 747

Zheng, Z., Flynn, C., Gould, A., Bahcall, J. N., \& Salim, S. 2001, ApJ, 555, 393 